\title{
THE VOLUME-PRESERVING MOTION BY MEAN CURVATURE AS AN ASYMPTOTIC LIMIT OF REACTION-DIFFUSION EQUATIONS
}

\author{
$\mathrm{BY}$ \\ DMITRY GOLOVATY \\ Department of Mathematics, Carnegie Mellon University, Pittsburgh, PA
}

Abstract. The asymptotic behavior of a nonlocal Ginzburg-Landau equation

$$
u_{t}^{\varepsilon}=\Delta u^{\varepsilon}-\frac{1}{\varepsilon^{2}} W^{\prime}\left(u^{\varepsilon}\right)+\frac{1}{\varepsilon} g\left(u^{\varepsilon}\right) \lambda^{\varepsilon}
$$

is studied when the small parameter $\varepsilon$ tends to zero. Here a Lagrange multiplier $\lambda^{\varepsilon}$ is introduced into the equation to enforce the conservation of mass. An energy-estimates approach is used to show that a limiting solution can be characterized by moving interfaces. It is further shown that the asymptotic limit of solutions of the nonlocal Ginzburg-Landau equation is a weak solution of the nonlocal, mass-preserving mean curvature flow. The weak solutions are constructed within a framework of the theory of viscosity solutions. In addition, the results describing interactions between the interfaces are obtained.

1. Introduction. Let $\Omega$ be a bounded open domain in $\mathbf{R}^{n}$ with an outward normal vector $\mathbf{n}(x)$ where $x \in \partial \Omega$, and consider the following nonlocal reaction-diffusion equation:

$$
\begin{cases}u_{t}^{\varepsilon}=\Delta u^{\varepsilon}-\frac{1}{\varepsilon^{2}} f\left(u^{\varepsilon}\right)+\lambda^{\varepsilon}, & x \in \Omega, t>0, \\ \left.\frac{\partial u^{\varepsilon}}{\partial \mathbf{n}}\right|_{\partial \Omega}=0, & x \in \partial \Omega, \\ u^{\varepsilon}(x, 0)=\phi(x), & x \in \Omega, t=0 .\end{cases}
$$

Here $f(u)=W^{\prime}(u)$ and $W(u)$ is a double-well potential, $\phi \in C^{\infty}(\Omega)$ is a function satisfying the compatibility condition $\left.\frac{\partial \phi}{\partial \mathbf{n}}\right|_{\partial \varsigma \Omega}=0$, while

$$
\lambda_{\varepsilon}=\frac{1}{\varepsilon^{2}|\Omega|} \int_{\Omega} f\left(u^{\varepsilon}\right) d x
$$

and $|\Omega|$ is the volume of $\Omega$. The small parameter $\varepsilon$ is introduced in such a way so that the diffusion term in (1) is negligible compared to the reaction term, except maybe in a narrow, of the order $\varepsilon$, transition layer where gradients are large.

Received June 27, 1994.

1991 Mathematics Subject Classification. Primary 35K57, 35B40. 
This problem was proposed by Rubinstein and Sternberg in [1]. They observed that, at least formally, (1) is a particular regime of the viscous Cahn-Hilliard equation, introduced by Novick-Cohen in [2]

$$
\alpha u_{t}=\Delta\left(f(u)-\beta \Delta u+\nu u_{t}\right) .
$$

Then (1) corresponds to the case when $\alpha$ is much smaller than $\beta, \nu$, and 1 , while $u$ is subject to the boundary conditions

$$
\begin{gathered}
\left.\frac{\partial}{\partial \mathbf{n}}\left(f(u)-\beta \Delta u+\nu u_{t}\right)\right|_{\partial \Omega}=0, \\
\left.\frac{\partial u}{\partial \mathbf{n}}\right|_{\partial \Omega \Omega}=\mathbf{0} .
\end{gathered}
$$

On the other hand, if $\nu \ll \alpha, \beta, 1$, then (3) reduces to

$$
\alpha u_{t}=\Delta(f(u)-\beta \Delta u) .
$$

Thus (1) can be interpreted as a simpler alternative to the Cahn-Hilliard equation (4).

Assuming that $u^{\varepsilon}$ is a concentration of one component in a binary mixture, we can also view (1) as a model of phase separation in that mixture, with (2) representing the mass conservation law

$$
\int_{\Omega} u^{\varepsilon} d x=\text { const }
$$

By analyzing Eq. (1) using the method of matched asymptotic expansions and multiple time scales, Rubinstein and Sternberg found that the phases rapidly separate as $\varepsilon \rightarrow 0$ and that the propagation of the interfaces between these phases is a coarsening process depending in a nonlocal manner on mean curvature. In other words, the functions $u^{\varepsilon}$ converge in $L^{1}(\Omega \times[0, T])$ to some function $u$, where $u$ assumes values at the points of the minimum of the double-well potential $W$ almost everywhere in $\Omega \times[0, T]$. Then the boundary $\Gamma_{t}$ between the regions of constant $u$ evolves according to

$$
V=\text { mean curvature }+\lambda(t) \text { in } \Omega,
$$

where $\lambda(t)$ is such that the volume of the region enclosed by the interfaces $\Gamma_{t}$ is constant in time, and $V$ is the normal velocity of $\Gamma_{t}$. The pair $\left(\Gamma_{t}, \lambda(t)\right)$ is called a volume-preserving mean curvature flow with associated geometric PDE

$$
v_{t}-\left(\Delta v-\frac{\left(D^{2} v D v, D v\right)}{|D v|^{2}}\right)-\lambda(t)|D v|=0 \quad \text { in } \mathbf{R}^{n} \times(0, \infty) .
$$

The evolutionary properties of the flow (6) differ from those of the flow by mean curvature. Indeed, it is well known that the motion by mean curvature does not exhibit stationary fronts not connected to the boundary. In fact, every initially bounded hypersurface in $\mathbf{R}^{n}$ will shrink into points in finite time. By contrast, the nonlocal flow provides more interesting structure that allows for the formation of stationary interfaces as well as interaction effects. A simple example of the stationary interface is given by a 
single sphere evolving in $\mathbf{R}^{n}$ by the volume-preserving flow. The nonlocal effects, however, present some difficulties in finding global solutions of (6). So far, some existence results exist only for short times and smooth initial data ([25], [26]).

In 1992 Barles, Soner, and Souganidis [3] developed a rigorous approach for obtaining the asymptotic limits of reaction-diffusion equations using viscosity techniques. In particular, they conjectured that their approach can be successfully used for studying the asymptotic limit of (1) under certain restrictions on the behavior of the Lagrange multiplier $\lambda^{\varepsilon}(t)$ as $\varepsilon \rightarrow 0$.

The method of [3] is based on a level set approach to motion by mean curvature, as developed by Evans and Spruck in [19] and Chen, Giga, and Goto in [20]. It consists of studying the properties of the signed distance function to the front. For example, for the standard Allen-Cahn equation

$$
u_{t}^{\varepsilon}=\Delta u^{\varepsilon}-\frac{1}{\varepsilon^{2}} f\left(u^{\varepsilon}\right)
$$

the procedure of finding the asymptotic limit can be briefly outlined as follows (here $f(u)=W^{\prime}(u)$ and $W(u)$ is a double-well potential).

First, using the asymptotic limit of the appropriate initial data and following [19], one can determine the unique generalized solution of the geometric PDE

$$
v_{t}-\Delta v+\frac{\left(D^{2} v D v, D v\right)}{|D v|^{2}}=0
$$

associated with the motion by mean curvature

$$
V=K
$$

of the interface $\Gamma_{t}$. Here the generalized solution is taken in a viscosity sense, as introduced by Crandall and Lions (see [21] for details), and $\Gamma_{t}$ is a (zero) level set of the function $v(\cdot, t)$. Then $V$ is a normal velocity of the interface $\Gamma_{t}$ and $K$ is its mean curvature.

It turns out that due to the uniqueness of the generalized solution of (9), one can find the differential inequalities that a continuous function $z$ must satisfy in a viscosity sense in order for $z$ to be identified with the signed distance function to the front $\Gamma_{t}$.

The solution of the reaction-diffusion equation (8) can be represented as $u^{\varepsilon}=q\left(z^{\varepsilon} / \varepsilon\right)$, where $q$ is a travelling wave corresponding to the potential $W$. Then, by taking the asymptotic limit of the resulting equation for $z^{\varepsilon}$ and using the theory of viscosity solutions, one can show that the weak limits of $z^{\varepsilon}$ satisfy the differential inequalities, mentioned in the previous paragraph. It follows then that $z^{\varepsilon}$ converges to the signed distance function to the front $\Gamma_{t}$ uniformly on $[0, T]$, and that the asymptotic limit of solutions of (8) evolves by the mean curvature flow.

In this paper we provide a rigorous version of [1] for a model

$$
\begin{gathered}
u_{t}^{\varepsilon}=\Delta u^{\varepsilon}-\frac{1}{\varepsilon^{2}} W^{\prime}\left(u^{\varepsilon}\right)+\frac{1}{\varepsilon} h^{\prime}\left(u^{\varepsilon}\right) \lambda^{\varepsilon}, \\
\int_{\Omega} h\left(u^{\varepsilon}(\cdot, t)\right) d x=\text { const },
\end{gathered}
$$


similar to the one studied by Rubinstein and Sternberg, using some of the techniques developed in [3], as well as the estimates found in [12]. For the precise definitions of the functions involved in this equation the reader is referred to the next section. This model can be obtained as a formal limit of the phase field equations for solidification (see, e.g., $[22])$

$$
\left\{\begin{array}{l}
u_{t}^{\varepsilon}=\Delta u^{\varepsilon}-\frac{1}{\varepsilon^{2}} W^{\prime}\left(u^{\varepsilon}\right)+\frac{1}{\varepsilon} h^{\prime}\left(u^{\varepsilon}\right) \theta, \\
\alpha \theta_{t}+\frac{1}{\varepsilon} h^{\prime}\left(u^{\varepsilon}\right) u_{t}^{\varepsilon}=\alpha^{-1} \Delta \theta,
\end{array}\right.
$$

as $\alpha \rightarrow 0$. Here the latent heat $\left(\frac{1}{\varepsilon} h^{\prime}\right)$ depends on an order parameter $u^{\varepsilon}$ in agreement with some of the recently developed thermodynamically consistent models, such as [23] or [24]. Then the model studied in [1] corresponds to the case when $h^{\prime}(u) \equiv 1$.

First, we obtain in Sec. 3 that, under the mass constraint, the nonlocal GinzburgLandau equation (12) is a gradient flow for the same energy functional as its local counterpart,

$$
E^{\varepsilon}\left[u^{\varepsilon}\right]:=\int_{\Omega}\left(\frac{\varepsilon}{2}\left|D u^{\varepsilon}\right|^{2}+\frac{1}{\varepsilon} W\left(u^{\varepsilon}\right)\right) d x .
$$

Then, using the energy type estimates and compactness arguments, we establish that for appropriately chosen initial data, the limiting solution of (10) indeed describes the phase separation. That is, the functions $u^{\varepsilon}$ converge in $L^{1}(\Omega \times[0, T])$ to some function $v \in B V(\Omega \times[0, T])$, where $v$ assumes values at the points of the minimum of the doublewell potential $W$ almost everywhere in $\Omega \times[0, T]$. The dependence of the limiting solution on time can therefore be characterized by the evolution of interfaces separating distinct phases.

It turns out that the asymptotic limit of the Lagrange multiplier $\lambda^{\varepsilon}$ as $\varepsilon \rightarrow 0$ depends on the asymptotic limit of the energy functional $E^{\varepsilon}$, evaluated along solution trajectories $u^{\varepsilon}$. We prove that $E^{\varepsilon}\left[u^{\varepsilon}\right]$ converges in $L^{1}[0, T]$ to a function $E$, where $E:[0, T] \rightarrow[0, \infty)$ is nonincreasing for any $T>0$.

Using the travelling wave representation of the solution $u^{\varepsilon}=: q\left(z^{\varepsilon} / \varepsilon\right)$, we obtain some additional bounds on $z^{\varepsilon}$ and its derivatives. Then, concentrating our attention on a radial domain $(\Omega=\{|x| \leq R\})$ and radially symmetric initial data, we apply a viscosity method based on the ideas of [3] to show that $z^{\varepsilon}$ converges to the signed distance function to the interface (Sec. 6.1) and establish the asymptotic behavior of the Lagrange multiplier $\lambda^{\varepsilon}$ (Sec. 6.2). Our analysis also relies on the estimates, similar to the ones obtained in [12]. Here we place some additional restrictions on the initial data in order to simplify the presentation of our method. They allow us to avoid dealing with possible nucleation as well as interactions of interfaces, at least locally in time. These additional assumptions are removed later, in Sec. 7 .

Next, in Sec. 6.3 we determine the limiting equations governing the motion of interfaces and establish the validity of the mass conservation law for $v$. The convergence of nonlocal reaction-diffusion equations to a nonlocal mean curvature flow is shown directly using viscosity methods, rather than applying the technique from [3]. This difference in approach is necessary in part because of the lack (to this date) of the global existence and uniqueness result for (7) along the lines of [19]. As we already mentioned above, this result is an important part of the method in [3]. 
Finally, in Sec. 7, we return to the case of the general initial data. To proceed, we introduce the concepts of a "ghost" and a "regular" interface. To illustrate these definitions formally for the radially symmetric problem in $\mathbf{R}^{2}$, suppose that $\Omega$ is a disk of radius $R$. Let $r \in[0, R]$ be such that in some neighborhood of $r$, the asymptotic limit $v$ of solutions of the Ginzburg-Landau equations is equal to $u_{1}$ to the right of $r$ and to $u_{2}$ to the left of $r$, where $u_{1}$ and $u_{2}$ are constants. Then we will say that there is a "regular" interface at $r$ if $u_{1} \neq u_{2}$ and that there is a "ghost" interface at $r$ if $u_{1}=u_{2}=u$ but $v(r) \neq u$. In this formal setting one can think of the "ghost" interface as a coupled pair of "regular" interfaces with the jumps of the same size and the opposite sign. In the remainder of the introduction we will simply use the word "interface" when stating the results that hold true for both types of interfaces. We will label interfaces by their $r$-coordinates.

When studying the behavior of a solution $u^{\varepsilon}$ of (10) near a given interface $r$, we find that $u^{\varepsilon}$ has a fixed number $m$ of "jumps" in an $o(1)$-neighborhood of $r$ uniformly in $\varepsilon$. Then it is customary to say that the multiplicity of the interface $r$ is $m$. For example, using our observation in the previous paragraph, one can see that the multiplicity of a "ghost" interface is greater than or equal to two and the multiplicity of a "regular" interface is greater than or equal to one. Our analysis would not require us to know the multiplicity of "regular" interfaces in order to determine their motion. The structure of interfaces will be, however, taken into account when we describe possible interactions between interfaces. The way to determine the multiplicity of an interface within the framework of our method is outlined in Sec. 7.1.

We show that for the appropriately chosen initial data, the positions of interfaces are continuous in time, and that no new interfaces can nucleate for any $t>0$ at the center of the domain $\Omega$, as well as in the interior of the set, which complements the set of interfaces in $\Omega$. This, along with the uniform boundedness of the energy allows us to modify the method of Sec. 6 to show that the asymptotic limit of the nonlocal Ginzburg-Landau equation is a weak solution of the nonlocal mass-preserving mean curvature flow.

Finally, we consider the interactions between interfaces. The "regular" interfaces moving by the nonlocal mean curvature flow might either collide with each other or shrink into the center of the domain $\Omega$. We show that "ghost" interfaces that appear after pairwise collisions of "regular" interfaces should disappear. Then, since no new interfaces can nucleate for any $t>0$, all "regular" interfaces, except one, disappear during the flow. This process is known as coarsening. Therefore, for large times, the asymptotic limit of solutions of the nonlocal Ginzburg-Landau equation can be characterized by a single stationary "regular" interface such that

$$
\int_{\Omega} v(x, t) d x=\int_{\Omega} v(x, 0) d x
$$

We are not able to characterize the dynamics of "ghost" interfaces. However, the formal analysis suggests that, in general, "ghost" interfaces do not exist, except for finitely many times $t \in[0, T]$. The same should be true for the multiplicity of interfacesit should not exceed one for all but finitely many $t \in[0, T]$. To prove these facts rigorously would require us to obtain the estimates on the limiting behavior of solutions of (10) that are much sharper than the ones found in [12]. 
For the reason of notational simplicity some of our analysis is done for $\Omega \subset \mathbf{R}^{2}$. However, our results can easily be extended to the spaces of higher dimensions.

Observe that our method can be, in principle, applied to (1). Then the analysis will become significantly more complicated, since the travelling wave representation for solutions $u^{\varepsilon}$ of (1) will depend explicitly on time.

After this work was completed we discovered that similar results were obtained in [4] by Bronsard and Stoth for the model (1). Their analysis is based on "energy methods" combined with a priori estimates of Stoth [12], the latter being used to approximate the solution by the first two terms of an asymptotic expansion. They obtained that for (1) the "regular" interfaces evolve by nonlocal flow, while "ghost" interfaces move by their mean curvature ("true" and "phantom" interfaces in the terminology of [4]). On the other hand, for the problem (10) there are formal indications that "ghost" interfaces cannot exist. In addition there is no nucleation of the new interfaces in our model for any $t>0$, while in [4] it cannot be ruled out for the times past the first geometric singularity of the nonlocal flow.

I would like to express a deep gratitude to my advisor, H. Mete Soner, for his encouragement, patience, and advice. I also wish to thank Irene Fonseca, Robert Jerrard, and Barbara Stoth for their comments on a draft of this paper.

2. Formulation of the problem. As we have mentioned already, we work with a model slightly different from (1). This is motivated in part by the use of a travelling wave representation for solutions of $(1)$, within the framework of the distance function approach for finding the asymptotic limit of the reaction-diffusion equations. Suppose that

$$
\begin{aligned}
W(u) & =\frac{1}{2}\left(u^{2}-1\right)^{2}, & & f(u)=W^{\prime}(u)=2 u\left(u^{2}-1\right), \\
h(u) & =2\left(\frac{u^{3}}{3}-u\right), & g(u) & =h^{\prime}(u)=2\left(u^{2}-1\right) .
\end{aligned}
$$

The choice of $W(u)$ and $h(u)$ is motivated purely by the simplicity of the calculations. Our results can easily be extended to an arbitrary double-well potential $W$ if we let, for example, $g(u)=\sqrt{2 W(u)}$. Consider now the following problem:

$$
\begin{cases}u_{t}^{\varepsilon}=\Delta u^{\varepsilon}-\frac{1}{\varepsilon^{2}} f\left(u^{\varepsilon}\right)+\frac{1}{\varepsilon} g\left(u^{\varepsilon}\right) \lambda^{\varepsilon}, & x \in \Omega, t>0, \\ \left.\frac{\partial u^{\varepsilon}}{\partial \mathbf{n}}\right|_{\partial \Omega}=0, & x \in \partial \Omega, \\ u^{\varepsilon}(x, 0)=\phi^{\varepsilon}(x), & x \in \Omega, t=0 .\end{cases}
$$

Here $\Omega$ and $\mathbf{n}$ are as before, $\phi^{\varepsilon} \in C^{\infty}(\Omega)$ for all $\varepsilon>0$, and $\lambda^{\varepsilon}$ is chosen in such a way that

$$
\int_{\Omega} h\left(u^{\varepsilon}\right) d x=\text { const . }
$$

The initial data are assumed to satisfy

$$
\begin{gathered}
E^{\varepsilon}\left[\phi^{\varepsilon}\right] \leq M \quad \text { for all } \varepsilon>0, \\
\phi^{\varepsilon} \rightarrow \phi \quad \text { in } L^{1}(\Omega), \\
\left|\phi^{\varepsilon}(x)\right| \leq 1, \quad x \in \Omega,
\end{gathered}
$$


where $M>0$ is a constant independent of $\varepsilon$ and $E^{\varepsilon}$ is the energy functional defined in (11). We will also assume that $\phi$ is neither identically 1 nor is it identically -1 almost everywhere in $\Omega$.

Multiplying Eq. (12) by $g\left(u^{\varepsilon}\right)$, integrating over $\Omega$ and using (13) we obtain

$$
\lambda^{\varepsilon}(t)=\varepsilon \cdot \frac{\int_{\Omega}\left[g^{\prime}\left(u^{\varepsilon}\right)\left|D u^{\varepsilon}\right|^{2}+\frac{1}{\varepsilon^{2}} f\left(u^{\varepsilon}\right) g\left(u^{\varepsilon}\right)\right] d x}{\int_{\Omega} g^{2}\left(u^{\varepsilon}\right) d x} .
$$

Then, using (15) and the fact that

$$
\begin{gathered}
g^{2}(u)=4\left(u^{2}-1\right)^{2}=8 W(u), \\
g^{\prime}(u)=4 u \\
f(u) g(u)=4 u\left(u^{2}-1\right)^{2}=8 u W(u),
\end{gathered}
$$

we have

$$
\lambda^{\varepsilon}(t)=\frac{\int_{\Omega} u^{\varepsilon}\left[\frac{\varepsilon}{2}\left|D u^{\varepsilon}\right|^{2}+\frac{1}{\varepsilon} W\left(u^{\varepsilon}\right)\right] d x}{\int_{\Omega} W\left(u^{\varepsilon}\right) d x} .
$$

The next theorem provides a travelling wave representation of $u^{\varepsilon}$.

Theorem 1. Suppose that $u^{\varepsilon}$ is a solution of (12) and that (14.c) is satisfied. Then $\left|u^{\varepsilon}(x, t)\right| \leq 1$ on $\bar{\Omega} \times[0, T]$.

Proof. The proof follows easily from the standard application of the maximum principle since $u_{0}= \pm 1$ are solutions of (12). Indeed suppose, for example, that $\left(x_{0}, t_{0}\right)$ is such that

$$
\max _{x \in \bar{\Omega}} u^{\varepsilon}\left(x, t_{0}\right)=u^{\varepsilon}\left(x_{0}, t_{0}\right)=1 \quad \text { and } \quad u_{t}^{\varepsilon}\left(x_{0}, t_{0}\right)>0 .
$$

Then $D u^{\varepsilon}\left(x_{0}, t_{0}\right)=0$ and $\Delta u^{\varepsilon}\left(x_{0}, t_{0}\right) \leq 0$. Substituting into (12) and using (14.c) one obtains a contradiction.

As in [1], we remark that since a local solution to (12) exists by a fixed point argument, using the previous theorem this local solution can be extended to a global one.

Now set $q:=\tanh$ and let $u^{\varepsilon}=: q\left(z^{\varepsilon} / \varepsilon\right)$. By the properties of the hyperbolic tangent, $q^{\prime}=1-q^{2}$ and $q^{\prime \prime}=-2 q q^{\prime}$. Hence, since $q^{\prime}>0,(12)$ and (16) take the form:

$$
\begin{gathered}
z_{t}^{\varepsilon}-\Delta z^{\varepsilon}+2 \lambda^{\varepsilon}(t)+\frac{2 u^{\varepsilon}}{\varepsilon}\left(\left|D z^{\varepsilon}\right|^{2}-1\right)=0, \\
\lambda^{\varepsilon}(t)=\frac{1}{\varepsilon} \cdot \frac{\int_{\Omega} q\left(z^{\varepsilon} / \varepsilon\right)\left(q^{\prime}\left(z^{\varepsilon} / \varepsilon\right)\right)^{2}\left(1+\left|D z^{\varepsilon}\right|^{2}\right) d x}{\int_{\Omega}\left(q^{\prime}\left(z^{\varepsilon} / \varepsilon\right)\right)^{2} d x},
\end{gathered}
$$

where $z^{\varepsilon}$ satisfies Neumann boundary conditions while $z^{\varepsilon}(\cdot, 0)=: \alpha^{\varepsilon} \in C^{\infty}(\Omega)$ and $\phi^{\varepsilon}(x)=q\left(\alpha^{\varepsilon}(x) / \varepsilon\right)$. We will assume, in addition to (14.a)-(14.c), that

$$
\left|D \alpha^{\varepsilon}\right| \leq 1, \quad \alpha^{\varepsilon} \rightarrow d_{0}(\cdot) \text { uniformly in } \Omega
$$

where $d_{0}(\cdot)$ is the signed distance to the set $\partial\{\phi(x)=1\}$. 
3. Energy estimates. Recall that

$$
E^{\varepsilon}\left[u^{\varepsilon}\right]=\int_{\Omega}\left(\frac{\varepsilon}{2}\left|D u^{\varepsilon}\right|^{2}+\frac{1}{\varepsilon} W\left(u^{\varepsilon}\right)\right) d x .
$$

Multiplying Eq. (12) by $u_{t}^{\varepsilon}$, integrating over $\Omega$, and using (13) we obtain

$$
E^{\varepsilon}\left[u^{\varepsilon}\right](t)=E^{\varepsilon}\left[u^{\varepsilon}\right](0)-\varepsilon \int_{0}^{t} \int_{\Omega}\left(u_{t}^{\varepsilon}\right)^{2} d x d t
$$

As a direct consequence of this equality one establishes

Theorem 2. Assume that (12), (14.a)-(14.b) hold. Then

(a)

$$
\sup _{t \geq 0} E^{\varepsilon}\left[u^{\varepsilon}\right](t) \leq M
$$

(b)

$$
\sup _{t \geq 0} \int_{\Omega}\left(\left(u^{\varepsilon}\right)^{2}-1\right)^{2} d x \leq 2 M \varepsilon
$$

(c)

$$
\varepsilon \int_{t_{1}}^{t_{2}} \int_{\Omega}\left(u_{t}^{\varepsilon}\right) d x d t=E^{\varepsilon}\left[u^{\varepsilon}\right]\left(t_{1}\right)-E^{\varepsilon}\left[u^{\varepsilon}\right]\left(t_{2}\right) \text { for } t_{2}>t_{1} .
$$

Also, we adopt the following compactness result from Bronsard and Kohn [6].

Theorem 3. Assume that (12), (14.a)-(14.b) are satisfied. Then for any sequence of $\varepsilon$ 's tending to zero there exists a subsequence $\varepsilon_{j}$ such that the $\operatorname{limit}_{\lim _{\varepsilon_{j} \rightarrow 0}} u^{\varepsilon_{j}}(x, t)=v(x, t)$ exists for a.e. $(x, t) \in \Omega \times(0, \infty)$. The function $v$ takes only the values \pm 1 , and there are positive constants $C$ and $C_{1}$ depending only on $M$ such that

$$
\begin{gathered}
\int_{\Omega}\left|v\left(x, t_{1}\right)-v\left(x, t_{2}\right)\right| d x \leq C\left|t_{2}-t_{1}\right|^{\frac{1}{2}}, \quad \text { for any } t_{2}, t_{1}>0 \\
\sup _{t \geq 0} \int_{\Omega}|D v(x, t)| d x \leq C_{1}
\end{gathered}
$$

and the initial value of $v$ is the limit of the initial data for $u^{\varepsilon}$ a.e. in $x$,

$$
\lim _{t \rightarrow 0} v(x, t)=\phi(x)
$$

Set

$$
E^{0}[u]:= \begin{cases}K \cdot \operatorname{Per}_{\Omega}(\{u=1\}), & \text { if } u(x) \in\{-1,1\} \text { a.e. in } \Omega \\ \infty, & \text { otherwise }\end{cases}
$$

where $K=\int_{-1}^{1} \sqrt{2 W(s)} d s$ (in our case $K=4 / 3$ ) and $\operatorname{Per}_{\Omega}(A)$ is a perimeter of $A$ in $\Omega$ (for the definition of perimeter see, e.g., [5]). It turns out that $E^{0}$ is a $\Gamma\left(L^{1}(\Omega)\right.$ )-limit of $E^{\varepsilon}$ (see, e.g., [7], [8], or [9]). In other words, the following holds: 
THEOREM 4. Let $E^{\varepsilon}$ and $E^{0}$ be as above.

(1) If $v^{\varepsilon} \rightarrow v^{0}$ in $L^{1}(\Omega)$ then

$$
\liminf _{\varepsilon \rightarrow 0} E^{\varepsilon}\left[v^{\varepsilon}\right] \geq E^{0}\left[v^{0}\right]
$$

(2) For any $v^{0} \in L^{1}(\Omega)$ there exists a family $\left(v^{\varepsilon}\right)$ such that $v^{\varepsilon} \rightarrow v^{0}$ in $L^{1}(\Omega)$ and

$$
\lim _{\varepsilon \rightarrow 0} E^{\varepsilon}\left[v^{\varepsilon}\right]=E^{0}\left[v^{0}\right]
$$

We can also obtain some additional information on the limit of $E^{\varepsilon}\left[u^{\varepsilon}\right]$ as a function of $t$. Fix $T>0$. Being monotone on $[0, T]$, each of the functions $E^{\varepsilon}\left[u^{\varepsilon}\right]$ belongs also to $B V([0, T])$, and by (14.a) and (21) the set $\left\{E^{\varepsilon}\left[u^{\varepsilon}\right]\right\}_{\varepsilon>0}$ is uniformly bounded in the $B V$-norm, hence is relatively compact in $L^{1}([0, T])$. Moreover, the following theorem holds $([17])$ :

THEOREM 5. Let $F$ be an infinite family of decreasing functions, defined on an interval $[0, T]$. If all functions of the family are bounded by the same number,

$$
|f(x)| \leq K, \quad f \in F, 0 \leq x \leq T,
$$

then there is a sequence of functions $\left\{f_{n}\right\}_{n \in \mathbf{N}}$ in $F$ that converges to a decreasing function $g$ at every point of $[a, b]$.

Therefore, there exists a subsequence $\left\{E^{\varepsilon_{j}}\left[u^{\varepsilon_{j}}\right]\right\}_{n \in \mathbf{N}}$ and a bounded, monotone decreasing, nonnegative function $E$ such that $E^{\varepsilon_{j}}\left[u^{\varepsilon_{j}}\right]$ converges to $E$ pointwise at every point of $[0, T]$. It will be shown later that $E^{0}[v](t)=E(t)$ everywhere, except maybe at finitely many $t$ 's.

Since $E$ is monotone, it is well known that a set of points where $E$ is discontinuous is at most countable, and that both left- and right-hand limits of $E$ exist at every point of $(0, T)$. This enables us to prove the next theorem.

THEOREM 6. Let $E^{\varepsilon_{j}}\left[u^{\varepsilon_{j}}\right]$ and $E$ be as above. If $t$ is a point of continuity of $E$, then

$$
\varlimsup_{\substack{j \rightarrow \infty \\ s \rightarrow t}}\left|E^{\varepsilon_{j}}\left[u^{\varepsilon_{j}}\right](s)-E(t)\right|=0 .
$$

Proof. Let $t$ be a point of continuity of $E$. Set

$$
a=\varlimsup_{\substack{j \rightarrow \infty \\ s \uparrow t}}\left|E^{\varepsilon_{j}}\left[u^{\varepsilon_{j}}\right](s)-E(t)\right| .
$$

Suppose that $a>0$. Then for sufficiently small $\varepsilon$ we can choose two subsequences $\left\{s_{j}\right\}_{j \in \mathbf{N}}$ and $\left\{\varepsilon_{j}\right\}_{j \in \mathbf{N}}$ such that $\left|E^{\varepsilon_{j}}\left[u^{\varepsilon_{j}}\right]\left(s_{j}\right)-E(t)\right| \geq \frac{a}{2}$ where $s_{j} \uparrow t$ and $\varepsilon_{j} \rightarrow 0$ as $j \rightarrow \infty$. Since both $E^{\varepsilon_{j}}\left[u^{\varepsilon_{j}}\right]$ and $E$ are monotone decreasing we obtain for any fixed $j \geq 1$ that

$$
E\left(s_{j}\right)=\lim _{k \rightarrow \infty} E^{\varepsilon_{k}}\left[u^{\varepsilon_{k}}\right]\left(s_{j}\right) \geq \varlimsup_{k \rightarrow \infty} E^{\varepsilon_{k}}\left[u^{\varepsilon_{k}}\right]\left(s_{k}\right) \geq E(t)+\frac{a}{2} .
$$

Therefore for any $j \geq 1$, we find that $E\left(s_{j}\right) \geq E(t)+a / 2$. But since $E$ is continuous at $t, \lim _{j \rightarrow \infty} E\left(s_{j}\right)$ exists and is greater than $E(t)+a / 2$. Thus $E$ has a jump at $t$, which contradicts the fact that $t$ is a continuity point of $E$. One can show that

$$
\varlimsup_{\substack{j \rightarrow \infty \\ s \downarrow t}}\left|E^{\varepsilon_{j}}\left[u^{\varepsilon_{j}}\right](s)-E(t)\right|=0
$$

as well, and then (31) follows. 
4. Additional estimates: The radial case. Interfaces. We now restrict our attention to the radial case $\Omega=\left\{x \in \mathbf{R}^{n}:|x|<R\right\}$. For the reason of notational simplicity we will assume that $n=2$ unless specified otherwise. Our results can be immediately extended to higher dimensions. From now on all functions will be assumed radially symmetric. The model (12) takes the form

$$
\begin{gathered}
u_{t}^{\varepsilon}=u_{r r}^{\varepsilon}+\frac{1}{r} u_{r}^{\varepsilon}-\frac{1}{\varepsilon^{2}} f\left(u^{\varepsilon}\right)+\frac{1}{\varepsilon} g\left(u^{\varepsilon}\right) \lambda^{\varepsilon}, \quad(r, t) \in \Omega_{T} \\
u_{r}^{\varepsilon}(t, 0)=u_{r}^{\varepsilon}(t, R)=0
\end{gathered}
$$

for $u^{\varepsilon}(r, t)$;

$$
\begin{gathered}
z_{t}^{\varepsilon}-z_{r r}^{\varepsilon}-\frac{1}{r} z_{r}^{\varepsilon}+2 \lambda^{\varepsilon}(t)+\frac{2 u^{\varepsilon}}{\varepsilon}\left(\left|z_{r}^{\varepsilon}\right|^{2}-1\right)=0, \quad(r, t) \in \Omega_{T} \\
z_{r}^{\varepsilon}(t, 0)=z_{r}^{\varepsilon}(t, R)=0
\end{gathered}
$$

for $z^{\varepsilon}(r, t)$. Here $\Omega_{T}:=[0, R] \times[0, T]$. For now we will suppose in addition to (19) that

$d_{0}(\cdot)$ has finitely many distinct zeroes $r_{i}(0), i=1, \ldots, N$ in $(0, R)$, $z_{r}^{\varepsilon}(\cdot, 0)$ has $N-1$ distinct zeroes $s_{i}^{\varepsilon}(0), i=1, \ldots, N-1$ in $(0, R)$,

$$
\begin{gathered}
z_{r r}^{\varepsilon}\left(s_{i}^{\varepsilon}(0), 0\right) \neq 0, i=1, \ldots, N-1 \text { for any } \varepsilon>0, \\
E^{\varepsilon}\left[\phi^{\varepsilon}\right] \rightarrow \frac{4}{3} \operatorname{Per}_{\Omega}(\{\phi=1\}) \text { as } \varepsilon \rightarrow 0 .
\end{gathered}
$$

We make these assumptions in order to simplify the presentation. They will be removed later in Sec. 7 when collisions between the interfaces will be discussed. Until then we will use the following definition.

Definition 1. Let $\alpha \in B V([0, R])$ be such that $\alpha(r) \in\{-1,1\}$ a.e. A point $r_{0} \in$ $(0, R)$ will be called an interface provided there exists $\delta>0$ such that $\alpha(r)=+1(-1)$ a.e. for $r \in\left(r_{0}-\delta, r_{0}\right)$ and $\alpha(r)=-1(+1)$ a.e. on $r \in\left(r_{0}, r_{0}+\delta\right)$. We denote the set of these points by $\Gamma$.

Observe that $r_{0}$ is an interface only if it belongs to the support of the Radon measure $|D \alpha|$, where $D \alpha$ is the gradient of $\alpha$ in the sense of distributions. Also, when (a)-(d) hold, the set $\partial\{\phi(x)=1\}$ consists of $N$ circular interfaces with radii $r_{i}(0), i=1, \ldots, N$.

Our assumptions lead us to another series of estimates:

Theorem 7. Assume that (19) holds. Then $\left|z_{r}^{\varepsilon}(r, t)\right| \leq 1$ on $\Omega_{T}$ for every $\varepsilon>0$.

Proof. From (34) we have that $w^{\varepsilon}:=z_{r}^{\varepsilon}$ satisfies the following problem:

$$
w_{t}^{\varepsilon}-w_{r r}^{\varepsilon}-\frac{1}{r} w_{r}^{\varepsilon}+\frac{1}{r^{2}} w^{\varepsilon}+\frac{4 q}{\varepsilon} w^{\varepsilon} w_{r}^{\varepsilon}+\frac{2 q^{\prime}}{\varepsilon^{2}} w^{\varepsilon}\left(\left(w^{\varepsilon}\right)^{2}-1\right)=0,
$$

where $q$ and $q^{\prime}$ are evaluated at $z^{\varepsilon} / \varepsilon$. At the same time:

$$
\begin{array}{ll}
w^{\varepsilon}(0, t)=w^{\varepsilon}(R, t)=0, & t \in(0, T), \\
\left|w^{\varepsilon}(r, 0)\right| \leq 1, & r \in(0, R) .
\end{array}
$$

Now the conclusion follows immediately by the maximum principle. 
Corollary 1. For all $t \in[0, T]$ one has $\left|\varepsilon \lambda^{\varepsilon}(t)\right| \leq 2$.

Proof. From the previous theorem and by (18) we find that:

$$
\left|\varepsilon \lambda^{\varepsilon}(t)\right|=\frac{\left|\int_{\Omega} q\left(z^{\varepsilon} / \varepsilon\right)\left(q^{\prime}\left(z^{\varepsilon} / \varepsilon\right)\right)^{2}\left(1+\left|D z^{\varepsilon}\right|^{2}\right) d x\right|}{\int_{\Omega}\left(q^{\prime}\left(z^{\varepsilon} / \varepsilon\right)\right)^{2} d x} \leq \frac{2 \int_{\Omega}\left(q^{\prime}\left(z^{\varepsilon} / \varepsilon\right)\right)^{2} d x}{\int_{\Omega}\left(q^{\prime}\left(z^{\varepsilon} / \varepsilon\right)\right)^{2} d x} \leq 2 .
$$

REMARK 1. Since " $z^{\varepsilon}(x, t)=z^{\varepsilon}(r(x), t)$ " and $z^{\varepsilon}(x, t)$ is smooth in $\Omega \times[0, T]$ as a solution of (17), we conclude that $z_{r r}^{\varepsilon}$ and $\frac{1}{r} z_{r}^{\varepsilon}$ are bounded in $\Omega_{T}$ for every $\varepsilon>0$.

Theorem 8. Suppose that $w^{\varepsilon}(\cdot, 0)$ has $N-1$ zeroes in $(0, R)$ for any $\varepsilon>0$. Then $w^{\varepsilon}(\cdot, t)$ has at most $N-1$ zeroes in $(0, R)$ for all $t \in[0, T]$ and $\varepsilon>0$.

The proof is based on the following lemma:

Lemma 1. Denote by $s_{i}^{\varepsilon}(0), i=1, \ldots, N-2$ the position of the $i$ th interior zero of $w^{\varepsilon}(\cdot, 0)$. Then

(a) For each $i=1, \ldots, N-2$, there exists a continuous curve $s_{i}^{\varepsilon}(t)$ such that $w^{\varepsilon}\left(s_{i}^{\varepsilon}(t), t\right)$ $=0$ and $w^{\varepsilon}(r, t) \neq 0$ if $r \in\left(s_{i}^{\varepsilon}(t), s_{i+1}^{\varepsilon}(t)\right)$ for all $t \leq \tau^{\varepsilon}$ and $i=1, \ldots, N-2$. Here $\tau^{\varepsilon}$ is such that either $s_{i}^{\varepsilon}\left(\tau^{\varepsilon}\right)=0$ or $s_{i}^{\varepsilon}\left(\tau^{\varepsilon}\right)=R$ or $s_{i}^{\varepsilon}\left(\tau^{\varepsilon}\right)=s_{i \pm 1}^{\varepsilon}\left(\tau^{\varepsilon}\right)$ for some $i=1, \ldots, N-2$.

(b) Suppose that two or more curves intersect at $(r, t)=\left(s^{\varepsilon}, \tau^{\varepsilon}\right)$. If the number of the intersecting curves is odd, then for $t>\tau^{\varepsilon}$ there exists a single continuous curve originating at the point of intersection. If the number of the intersecting curves is even, there exist $\delta, \gamma>0$ depending on $\varepsilon$ such that $w^{\varepsilon}(r, t) \neq 0$ for all $(r, t) \in\left(s^{\varepsilon}-\delta, s^{\varepsilon}+\delta\right) \times$ $\left(\tau^{\varepsilon}, \tau^{\varepsilon}+\gamma\right)$.

(c) Let $s_{0}^{\varepsilon}(t) \equiv 0$ and $s_{N}^{\varepsilon}(t) \equiv R$. Then the result of part (a) can be extended for $i=0, \ldots, N-1$. Also if $\tau_{i}^{\varepsilon}$ is such that $s_{i}^{\varepsilon}\left(\tau_{i}^{\varepsilon}\right)=0$, then there exist $\delta, \gamma>0$ depending on $\varepsilon$ such that $w^{\varepsilon}(r, t)>0(<0)$ where $(r, t) \in(0, \delta) \times\left(\tau_{i}^{\varepsilon}, \tau_{i}^{\varepsilon}+\gamma\right)$. A similar result holds when $s_{i}^{\varepsilon}\left(\tau_{i}^{\varepsilon}\right)=R$.

A more general result for a solution of a parabolic equation was obtained by Angenent in $[10]$.

Proof of Lemma 1. The proof is based on a maximum principle.

(a) $w^{\varepsilon}$ is smooth because it is a solution of (37.a)-(37.c). Thus, from our assumption on $w^{\varepsilon}(\cdot, 0)$, we obtain by the implicit function theorem that smooth functions $s_{i}^{\varepsilon}(t)$, $i=1, \ldots, N-1$ exist for some small time, say $\tau^{\varepsilon}$. By the strict maximum principle and the smoothness of $w^{\varepsilon}$, the curves $s_{i}^{\varepsilon}$ must also remain continuous for $i=1, \ldots, N-2$ as functions of $t$ past the time $\tau^{\varepsilon}$. The sign of $w^{\varepsilon}$ follows by the strict maximum principle as well. Observe that since $w^{\varepsilon}$ is smooth, then for every $i=1, \ldots, N-2$ the curves $s_{i}^{\varepsilon}$ cannot terminate without intersecting either one of the adjacent curves $s_{i}^{\varepsilon}$ or hitting the boundary $r=0$ or $r=R$. Therefore, we let

$$
\tau^{\varepsilon}:=\sup \left\{t>0: 0 \neq s_{1}^{\varepsilon}(t) \neq \cdots \neq s_{N-2}^{\varepsilon}(t) \neq R\right\}
$$

(b) Suppose that the even number of curves, for example $s_{i}^{\varepsilon}$ and $s_{i+1}^{\varepsilon}$, intersect at $(r, t)=\left(s^{\varepsilon}, \tau^{\varepsilon}\right)$ for some $i \in\{1, \ldots, N\}$. For $t=t_{0}$ we have that $w^{\varepsilon}(r, t)>0(<0)$ on $\left(s_{i-1}^{\varepsilon}\left(\tau^{\varepsilon}\right), s_{i}^{\varepsilon}\left(\tau^{\varepsilon}\right)\right) \cup\left(s_{i}^{\varepsilon}\left(\tau^{\varepsilon}\right), s_{i+2}^{\varepsilon}\left(\tau^{\varepsilon}\right)\right)$ and $w^{\varepsilon}\left(s_{i}^{\varepsilon}\left(\tau^{\varepsilon}\right), \tau^{\varepsilon}\right)=0$. Then, if $t_{1}^{\varepsilon}>\tau^{\varepsilon}$ is such that $s_{i-1}^{\varepsilon}\left(t_{1}^{\varepsilon}\right) \neq s_{i+2}^{\varepsilon}\left(t_{1}^{\varepsilon}\right)$, the strict maximum principle implies that $w^{\varepsilon}(r, t)>0(<0)$ for $t \in\left(\tau^{\varepsilon}, t_{1}^{\varepsilon}\right)$ and $r \in\left(s_{i-1}^{\varepsilon}(t), s_{i+2}^{\varepsilon}(t)\right)$. A similar proof works when the odd number of curves intersect at a single point. 
(c) Consider, for example, the endpoint $r=0$. Let $t=\tau_{1}^{\varepsilon}$ be a time such that $s_{1}^{\varepsilon}\left(\tau_{1}^{\varepsilon}\right)=0$. Assume $t<\tau_{1}^{\varepsilon}$. One can easily check, following e.g. [11], that even though equation (37.a) has a singularity at $r=0$, the strict maximum principle applies inside the parabolic region bounded by $r=s_{1}^{\varepsilon}(t)$ from the right, by $r=s_{0}^{\varepsilon}(t)$, from the left, and by $t=0$ from below (since the solution $u^{\varepsilon}(\cdot, t)$ is smooth in $\Omega$ for any $t>0$, cf. Remark 1). As in (a), we have that $w^{\varepsilon}(r, t)$ is strictly positive (negative) for $r \in\left(s_{0}^{\varepsilon}(t), s_{1}^{\varepsilon}(t)\right)$ if $w^{\varepsilon}(r, 0)$ is strictly positive (negative) for $r \in\left(s_{0}^{\varepsilon}(0), s_{1}^{\varepsilon}(0)\right)$. After the time $\tau_{1}^{\varepsilon}$, using the strict maximum principle once again, we can apply the analysis as the one in part (b) to show that the curve $s_{i}^{\varepsilon}$ disappears when it crosses the boundary $r=0$ and no additional curves are created. The same results hold for $r=R$.

Proof of Theorem 8. Follows immediately from the previous lemma.

5. Asymptotic behavior of the Lagrange multiplier. We will use extensively the estimates below, due to Stoth [12], which are obtained by:

(a) multiplying Eq. (32) by $\varepsilon r u_{r}^{\varepsilon}$ and integrating in $r$ by parts from $y$ to $y_{1}$, where $y$ and $y_{1} \in[0, R]$, precisely

$$
\begin{aligned}
\varepsilon \int_{y}^{y_{1}} u_{r}^{\varepsilon} u_{t}^{\varepsilon} r d r-r & {\left.\left[\varepsilon \frac{\left(u_{r}^{\varepsilon}\right)^{2}}{2}-\frac{W\left(u^{\varepsilon}\right)}{\varepsilon}\right]\right|_{y} ^{y_{1}}-\int_{y}^{y_{1}}\left[\varepsilon \frac{\left(u_{r}^{\varepsilon}\right)^{2}}{2}+\frac{W\left(u^{\varepsilon}\right)}{\varepsilon}\right] d r } \\
& -\left[y_{1} h\left(u^{\varepsilon}\left(y_{1}\right)\right)-y h\left(u^{\varepsilon}(y)\right)-\int_{y}^{y_{1}} h\left(u^{\varepsilon}(r)\right) d r\right] \cdot \lambda^{\varepsilon}(t)=0
\end{aligned}
$$

(b) differentiating Eq. (32) in $t$, multiplying the result by $\varepsilon r u_{t}^{\varepsilon}$ and integrating in $r$ by parts over $[0, R]$ :

$$
\begin{aligned}
& \frac{1}{2} \frac{d}{d t}\left(\varepsilon \int_{0}^{R}\left(u_{t}^{\varepsilon}\right)^{2} r d r\right) \\
& \quad=-\varepsilon \int_{0}^{R}\left|\nabla u_{t}^{\varepsilon}\right|^{2} r d r-\frac{1}{\varepsilon} \int_{0}^{R}\left[f^{\prime}\left(u^{\varepsilon}\right)-\varepsilon \lambda^{\varepsilon} g^{\prime}\left(u^{\varepsilon}\right)\right]\left(u_{t}^{\varepsilon}\right)^{2} r d r
\end{aligned}
$$

REMARK 2. Multiplying (24) by $\varepsilon^{\alpha}$ for any $\alpha>0$ and using (22) we find that

$$
\varepsilon^{1+\alpha} \int_{0}^{R}\left(u_{t}^{\varepsilon}\right)^{2} r d r \rightarrow 0 \quad \text { in } L^{1}([0, T]) \text { as } \varepsilon \rightarrow 0
$$

Then passing, if necessary, to a subsequence

$$
\varepsilon^{1+\alpha} \int_{0}^{R}\left(u_{t}^{\varepsilon}\right)^{2} r d r \rightarrow 0 \text { a.e. } t \in[0, T] .
$$

REMARK 3. After multiplying estimate (39) by $2 \varepsilon^{2}$, integrating it over $\left[t, t_{1}\right]$ for any $t, t_{1} \in[0, T]$, and using $(22),(24)$ and Corollary 1 we get

$$
\left.\varepsilon^{3} \int_{0}^{R}\left(u_{t}^{\varepsilon}\right)^{2} r d r\right|_{t} ^{t_{1}} \leq C\left[E^{\varepsilon}\left(t_{1}\right)-E^{\varepsilon}(t)\right]
$$


where $C$ is a constant independent of $\varepsilon$.

REMARK 4 . Now multiply the estimate (38) by $\varepsilon$. Then the first term can be bounded by

$$
\begin{aligned}
\varepsilon^{2}\left|\int_{y}^{y_{1}} u_{r}^{\varepsilon} u_{t}^{\varepsilon} r d r\right| & \leq \varepsilon^{2}\left|\int_{0}^{R} u_{r}^{\varepsilon} u_{t}^{\varepsilon} r d r\right| \leq\left(\int_{0}^{R} \varepsilon\left(u_{r}^{\varepsilon}\right)^{2} r d r\right)^{\frac{1}{2}}\left(\int_{0}^{R} \varepsilon^{3}\left(u_{t}^{\varepsilon}\right)^{2} r d r\right)^{\frac{1}{2}} \\
& \leq M^{\frac{1}{2}}\left(\int_{0}^{R} \varepsilon^{3}\left(u_{t}^{\varepsilon}\right)^{2} r d r\right)^{\frac{1}{2}} \rightarrow 0 \text { a.e. } t \in[0, T]
\end{aligned}
$$

by Remark 2 . The third term in (38) when multiplied by $\varepsilon$, converges to zero by (22). Moreover, for each $t \in[0, T]$ we can choose $y$ and $y_{1} \in(0, R)$ arbitrarily close to each other such that, for example, $u^{\varepsilon}(y, t) \rightarrow-1$ and $u^{\varepsilon}\left(y_{1}, t\right) \rightarrow+1$. Choosing $y$ and $y_{1}$ to be the limits of integration in (38), one can easily see that the coefficient of $\varepsilon \lambda^{\varepsilon}$ approaches a nonzero constant as $\varepsilon \rightarrow 0$, while the term

$$
\left.\frac{1}{2} r\left[\left(q^{\prime}\right)^{2}\left(\left(z_{r}^{\varepsilon}\right)^{2}-1\right)\right]\right|_{y} ^{y_{1}}
$$

converges to zero. This implies that $\varepsilon \lambda^{\varepsilon} \rightarrow 0$ as $\varepsilon \rightarrow 0$ a.e. $t \in[0, T]$.

TheOREM 9. $\varepsilon \lambda^{\varepsilon} \rightarrow 0$ as $\varepsilon \rightarrow 0$ at the points of continuity of $E$.

Proof. Suppose that $t$ is a continuity point of $E$. According to Remark 2 we can pass to a subsequence (independent of $t$ ) such that in any small neighborhood of $t$ we can find $t_{1}$ for which $\varepsilon^{3} \int_{0}^{R}\left(u_{t}^{\varepsilon}\right)^{2} r d r \rightarrow 0$ at $t_{1}$ as $\varepsilon \rightarrow 0$. At the same time, by Remark 3 we have

$$
\left(\varepsilon^{3} \int_{0}^{R}\left(u_{t}^{\varepsilon}\right)^{2} r d r\right)(t) \leq\left(\varepsilon^{3} \int_{0}^{R}\left(u_{t}^{\varepsilon}\right)^{2} r d r\right)\left(t_{1}\right)+C\left[E^{\varepsilon}\left(t_{1}\right)-E^{\varepsilon}(t)\right]
$$

hence

$$
\varlimsup_{\varepsilon \rightarrow 0}\left(\varepsilon^{3} \int_{0}^{R}\left(u_{t}^{\varepsilon}\right)^{2} r d r\right)(t) \leq C\left[E\left(t_{1}\right)-E(t)\right] .
$$

The right-hand side of this expression can be made arbitrarily small by choosing $t_{1}$ close enough to $t$, which proves that the

$$
\lim _{\varepsilon \rightarrow 0}\left(\varepsilon^{3} \int_{0}^{R}\left(u_{t}^{\varepsilon}\right)^{2} r d r\right)(t)
$$

exists and is equal to zero. Then, using the same procedure as in Remark 4, we conclude that $\lim _{\varepsilon \rightarrow 0} \varepsilon \lambda^{\varepsilon}(t)=0$.

THEOREM 10. $\lim _{\substack{\varepsilon \rightarrow 0 \\ s \rightarrow t}} \varepsilon \lambda^{\varepsilon}(s)=0$ at the points of continuity of $E$.

Proof. Assume again that $t$ is a continuity point of $E$. Following the proof of the previous theorem and using $t$ as $t_{1}$, and $s$ as $t$, by Theorem 6 and Theorem 9 we obtain

$$
\varlimsup_{\substack{\varepsilon \rightarrow 0 \\ s \rightarrow t}}\left|\varepsilon \lambda^{\varepsilon}(s)\right| \leq C \varlimsup_{\substack{\varepsilon \rightarrow 0 \\ s \rightarrow t}}\left[\left(\varepsilon^{3} \int_{0}^{R}\left(u_{t}^{\varepsilon}\right)^{2} r d r\right)(t)+C_{1}\left[E^{\varepsilon}(s)-E^{\varepsilon}(t)\right]\right]=0 .
$$


6. Asymptotic behavior of reaction-diffusion equations. The small time. We start by considering in this section the asymptotic limit of the reaction-diffusion equations for the short time, and then continue by investigating in Sec. 7 the limiting behavior of our model as time progresses by allowing for disappearance of interfaces and their interactions.

6.1. Asymptotic limit of $z^{\varepsilon}$. By (29), (36.d), and Theorem 5 we have that

$$
\begin{aligned}
\frac{8 \pi}{3} \sum_{i=1}^{N} r_{i}(0) & =\frac{4}{3} \operatorname{Per}_{\Omega}(\{\phi=1\})=E(0) \geq E(t) & \text { for all } t>0, \\
& =\lim _{\varepsilon \rightarrow 0} E^{\varepsilon}(t) \geq E^{0}(t)=\frac{4}{3} \operatorname{Per}_{\Omega}(\{v(\cdot, t)=1\}) &
\end{aligned}
$$

where $r_{i}(0), i=1, \ldots, N$ are the positions of the interfaces (see Def. 1) at $t=0$. At the same time, by Theorem 3 ,

$$
\int_{0}^{R}|v(r, t)-\phi(r)| r d r \leq C t^{1 / 2}, \quad \text { for all } t>0 .
$$

Then, since $v$ and $\phi$ take the values \pm 1 a.e., we can prove the following simple lemma.

Lemma 2. Assume that for $t>0$,

$$
\int_{0}^{R}|v(r, t)-\phi(r)| r d r<l^{2} / 2
$$

where $l=\min \left\{r_{1}(0) ; R-r_{N}(0) ; r_{i+1}(0)-r_{i}(0), 1 \leq i \leq N-1\right\}$ is the smallest distance between two interfaces (see Def. 1) or between the interface and the boundary at time zero. Then there are at least $N$ interfaces at time $t$.

REMARK 5. A similar result holds in $\mathbf{R}^{n}, n>2$, although with a different constant.

Proof of Lemma 2. Let $\Theta:=\left\{\alpha \in B V([0, R]): \int_{0}^{R}\left|\alpha^{2}-1\right| d r=0, \alpha\right.$ has $N-1$ interfaces $\}$. We want to show that for $\alpha \in \Theta$,

$$
\int_{0}^{R}|\alpha(r)-\phi(r)| r d r>l^{2} / 2
$$

The proof can be done by using a simple geometric argument. By varying the positions of interfaces for $\alpha$, relative to the ones for $\phi$ and taking into account that $|\alpha(r)-\phi(r)|$ is equal to 0 or 2 a.e. on $[0, R]$, we find that

$$
\inf _{\alpha \in \Theta} \int_{0}^{R}|\alpha(r)-\phi(r)| r d r=l^{2}>l^{2} / 2 .
$$

Suppose now that $v(\cdot, t)$ has $N-1$ interfaces. Then $v(\cdot, t) \in \Theta$ and thus satisfies (45). But this contradicts the assumption of the lemma. Furthermore, it is easy to observe that if $\Theta$ contains $\alpha$ 's with less than $N-1$ interfaces, (46) will still hold. Then there are at least $N$ interfaces at time $t$.

Set $\tau=l^{4} /\left(4 C^{2}\right)$ with $C$ as in (44). The inequality (44) and the above lemma imply that if $t<\tau$, then there exist at least as many interfaces at time $t$ as there are at time zero. 
Theorem 11. There are exactly $N$ interfaces, $0<r_{1}(t)<\cdots<r_{N}(t)<R$, at the time $t$, such that $t<\tau$. Furthermore, $u^{\varepsilon}(\cdot, t)$ converges to +1 or -1 uniformly on

$$
\left\{\bigcup_{i=1}^{N-1}\left[r_{i}(t)+\delta, r_{i+1}(t)-\delta\right]\right\} \cup\left[\delta, r_{1}(t)-\delta\right] \cup\left[r_{N}(t)+\delta, R-\delta\right]
$$

as $\varepsilon \rightarrow 0$ for each $i=1, \ldots, N-1$ and any $\delta>0$.

Proof. (a) By Lemma 2 we know that there are at least $N$ interfaces. Suppose now that their number exceeds $N$, and it is equal to $N+K$. Let $r_{i}(t)$, where $1 \leq i \leq N+K$, be a position of the $i$ th interface at time $t<\tau$. Fix $i$ and choose a small $\delta>0$. We want to show that for $\varepsilon$ small enough, $u^{\varepsilon}(\cdot, t)$ has a zero inside $\left(r_{i}(t)-\delta, r_{i}(t)+\delta\right)$. Indeed, if this is not the case, then we can choose a subsequence $\left\{\varepsilon_{j}\right\}_{j \in \mathbf{N}}$ such that $u^{\varepsilon_{j}}(\cdot, t)>0$ $(<0)$ on $\left(r_{i}(t)+\delta, r_{i+1}(t)-\delta\right)$ for every $j$ large enough. Since $u^{\varepsilon_{j}}(\cdot, t)$ converges to $v(\cdot, t)$ in $L^{1}([0, R])$ we obtain that $v(\cdot, t)>0$ a.e. on $\left(r_{i}(t)-\delta, r_{i}(t)+\delta\right)$ which contradicts our definition of the interface. Thus, for $\varepsilon>0$ small, $u^{\varepsilon}(\cdot, t)$ has at least one zero near each of the $N+K$ interfaces and $u_{r}^{\varepsilon}(\cdot, t)$ has at least $N+K-1$ zeroes inside $(0, R)$ by Rolle's theorem. This contradicts Theorem 8 . Therefore, there are exactly $N$ interfaces at time $t$.

(b) Fix $\delta>0$ and $t<\tau$. By (23),

$$
\int_{\Omega}\left(1-\left|u^{\varepsilon}\right|\right) d x \leq 8 M \varepsilon
$$

for any $t \geq 0$. By (a) there exists $\varepsilon_{0}>0$ small, such that $u^{\varepsilon}(\cdot, t)$ has a zero inside $\left[r_{i}(t)-\frac{\delta}{2}, r_{i}(t)+\frac{\delta}{2}\right]$ for each $i=1, \ldots, N$ and $\varepsilon<\varepsilon_{0}$. It is easy to observe that $\left|u^{\varepsilon}\right|$ does not have local minima on

$$
\left\{\bigcup_{i=1}^{N-1}\left[r_{i}(t)+\delta, r_{i+1}(t)-\delta\right]\right\} \cup\left[\delta, r_{1}(t)-\delta\right] \cup\left[r_{N}(t)+\delta, R-\delta\right] .
$$

Then for every $\varepsilon<\varepsilon_{0}$ the minimum of $\left|u^{\varepsilon}\right|$ on each of the intervals $\left[r_{i}(t)+\delta, r_{i+1}(t)-\right.$ $\delta],\left[\delta, r_{1}(t)-\delta\right]$, or $\left[r_{N}(t)+\delta, R-\delta\right]$ occurs at one of the endpoints. Hence if $\left|u^{\varepsilon}\right|$ does not converge to +1 uniformly on $\left[r_{i}(t)+\delta, r_{i+1}(t)-\delta\right]$ for some $i=1, \ldots, N-1$, then

$$
\min \left(\liminf _{\varepsilon \rightarrow 0}\left|u^{\varepsilon}\left(r_{i}(t)+\delta, t\right)\right|, \liminf _{\varepsilon \rightarrow 0}\left|u^{\varepsilon}\left(r_{i+1}(t)-\delta, t\right)\right|\right)=: a<1 .
$$

Therefore,

$$
\frac{\delta(1-a)}{2} \leq \varlimsup_{\varepsilon \rightarrow 0} \int_{\Omega}\left(1-\left|u^{\varepsilon}\right|\right) d x .
$$

This contradicts (47).

We are now in a position to find the asymptotic limit of $z^{\varepsilon}$.

Theorem 12. Let $\tau$ be as above. Then $z^{\varepsilon}\left(\cdot, t_{0}\right) \rightarrow d\left(\cdot, t_{0}\right)$ uniformly as $\varepsilon \rightarrow 0$ if $t_{0} \in[0, \tau]$ is a continuity point of $E$. Here $d\left(\cdot, t_{0}\right)$ is a signed distance function to the set $\Gamma_{t}$ of interfaces. 
Proof. The proof is based on the method in [3] to which we refer the reader for a more detailed treatment. Let us first make the following definitions:

$$
\begin{aligned}
& z_{*}(r, t)=\liminf _{\substack{\varepsilon \rightarrow 0 \\
s \rightarrow t}} z^{\varepsilon}(r, s), \\
& z^{*}(r, t)=\limsup _{\substack{\varepsilon \rightarrow 0 \\
s \rightarrow t}} z^{\varepsilon}(r, s) .
\end{aligned}
$$

Then $z^{*}$ is an upper semicontinuous and $z_{*}$ is a lower semicontinuous function on $\Omega_{\tau}:=$ $[0, R] \times[0, \tau]$. Moreover, by Theorem 7 , both $z^{*}(\cdot, t)$ and $z_{*}(\cdot, t)$ are Lipschitz continuous for all $t>0$ with Lipschitz constant $L=1$.

(a) Let $\Psi \in C^{1}([0, R])$ be such that $\left(z^{*}-\Psi\right)\left(\cdot, t_{0}\right)$ has a maximum at $r=r_{0}$, where $r_{0} \in\left(r_{i}\left(t_{0}\right)+\delta, r_{i+1}\left(t_{0}\right)-\delta\right)$ for some fixed $i$ and $\delta$ and suppose that $u^{\varepsilon}\left(\cdot, t_{0}\right)$ converges to -1 on this interval. Since $q^{\prime}>0$ then $z^{\varepsilon}\left(\cdot, t_{0}\right)<0$ on the same interval if $\varepsilon$ is sufficiently small; therefore $z^{*}\left(\cdot, t_{0}\right) \leq 0$ by Theorem 11 and Theorem 3 .

Let

$$
g_{\alpha}(r, t)=\Psi(r)+\frac{\left(r-r_{0}\right)^{2}}{2 \gamma}+\frac{\left(t-t_{0}\right)^{2}}{2 \alpha}
$$

for $\alpha>0, \gamma>0$ small. Then, since $z^{*}$ is upper semicontinuous on a bounded domain, $z^{*}-g_{\alpha}$ has a global maximum at some $\left(r_{\alpha}, t_{\alpha}\right) \in \Omega_{\tau}$. By the construction of $g_{\alpha}$ and the boundedness of $z^{*}$ we have that $\left(r_{\alpha}, t_{\alpha}\right) \notin\{0, R\} \times[0, \tau] \cup[0, R] \times\{0, \tau\}$ for $\alpha$ and $\gamma$ sufficiently small. Furthermore, even though this maximum might not be strict, it is attained inside some small region, determined by the values of $\alpha$ and $\gamma$.

If we keep $\gamma$ fixed and let $\alpha \rightarrow 0$ then

$$
\begin{aligned}
\left(z^{*}-\Psi\right)\left(r_{0}, t_{0}\right) & =\left(z^{*}-g_{\alpha}\right)\left(r_{0}, t_{0}\right) \\
& \leq\left(z^{*}-g_{\alpha}\right)\left(r_{\alpha}, t_{\alpha}\right) \\
& \leq z^{*}\left(r_{\alpha}, t_{\alpha}\right)-\Psi\left(r_{\alpha}\right)-\frac{\left(r_{\alpha}-r_{0}\right)^{2}}{2 \gamma}-\frac{\left(t_{\alpha}-t_{0}\right)^{2}}{2 \alpha} .
\end{aligned}
$$

Since $\left(r_{\alpha}, t_{\alpha}\right)$ is contained in a bounded domain, we can choose a subsequence $\left\{\alpha_{k}\right\}_{k \in \mathbf{N}}$ such that $\left(r_{\alpha_{k}}, t_{\alpha_{k}}\right) \rightarrow\left(r_{1}, t_{1}\right) \in \Omega_{\tau}$. Then immediately $t_{1}=t_{0}$ and since

$$
\left(z^{*}-\Psi\right)\left(r_{0}, t_{0}\right) \leq z^{*}\left(r_{\alpha_{k}}, t_{\alpha_{k}}\right)-\Psi\left(r_{\alpha_{k}}, t_{\alpha_{k}}\right)
$$

by passing to the limit in $\alpha_{k}$ we obtain

$$
\begin{aligned}
\left(z^{*}-\Psi\right)\left(r_{0}, t_{0}\right) & \leq \limsup _{\alpha_{k} \rightarrow 0} z^{*}\left(r_{\alpha_{k}}, t_{\alpha_{k}}\right)-\Psi\left(r_{1}, t_{0}\right) \\
& \leq z^{*}\left(r_{1}, t_{0}\right)-\Psi\left(r_{1}, t_{0}\right) \leq\left(z^{*}-\Psi\right)\left(r_{0}, t_{0}\right)
\end{aligned}
$$

Therefore, $r_{1}=r_{0}$ and since any sequence contains a subsequence converging to the same limit, $\left(r_{\alpha}, t_{\alpha}\right) \rightarrow\left(r_{0}, t_{0}\right)$ as $\alpha \rightarrow 0$.

(b) Since $z^{*}-g_{\alpha}$ attains its maximum on some small subset $A$ of $\Omega_{\tau}$, by definition of $z^{*}$ we know that $z^{\varepsilon}-g_{\alpha}$ must attain a local maximum $\left(r_{\alpha}^{\varepsilon}, t_{\alpha}^{\varepsilon}\right)$ in some neighborhood of $A$, 
for sufficiently small $\varepsilon$ 's, passing, if necessary, to a subsequence $\left(r_{\alpha}^{\varepsilon}, t_{\alpha}^{\varepsilon}\right) \rightarrow\left(r_{\alpha}, t_{\alpha}\right) \in \bar{A}$. Then by (17) we have at $\left(r_{\alpha}^{\varepsilon}, t_{\alpha}^{\varepsilon}\right)$ that

$$
\frac{1}{\alpha}\left(t_{\alpha}^{\varepsilon}-t_{0}\right)-\Psi_{r r}-\frac{1}{\gamma}+2 \lambda^{\varepsilon}+\frac{2 u^{\varepsilon}}{\varepsilon}\left(\left|\Psi_{r}+\frac{1}{\gamma}\left(r_{\alpha}^{\varepsilon}-r_{0}\right)\right|^{2}-1\right) \leq 0 .
$$

Multiplying this estimate by $\varepsilon$ and letting $\varepsilon \rightarrow 0$ we obtain that by Theorem 11 and Theorem 3

$$
2 \liminf _{\substack{\varepsilon \rightarrow 0 \\ s \rightarrow t_{\alpha}}} \varepsilon \lambda^{\varepsilon}-2\left(\left|\Psi_{r}+\frac{1}{\gamma}\left(r_{\alpha}-r_{0}\right)\right|^{2}-1\right) \leq 0 \text { at }\left(r_{\alpha}, t_{\alpha}\right) .
$$

Now if we send $\alpha$ to zero, use (a) and Theorem 10, we arrive at $1-\Psi_{r}^{2}\left(r_{0}, t_{0}\right) \leq 0$. On the other hand, by Theorem 7 , if $\Psi \in C^{1}([0, R])$ is such that $\left(z^{*}-\Psi\right)\left(\cdot, t_{0}\right)$ has a minimum at $r=r_{0}$ then $1-\Psi_{r}^{2}\left(r_{0}, t_{0}\right) \geq 0$, hence $z^{*}\left(\cdot, t_{0}\right)$ is a viscosity solution of $1-|D z|^{2}=0$ in $\left(r_{i}\left(t_{0}\right)+\delta, r_{i+1}\left(t_{0}\right)-\delta\right)$ for arbitrary $\delta>0$ (since $z^{*}\left(\cdot, t_{0}\right)$ is Lipschitz continuous for a fixed $t_{0}$ ).

(c) Since

$$
z^{*}\left(r, t_{0}\right) \geq z_{*}\left(r, t_{0}\right)
$$

the fact that the interface is located at $r_{i}\left(t_{0}\right)$ requires that $z^{*}\left(r_{i}\left(t_{0}\right), t_{0}\right)=z_{*}\left(r_{i}\left(t_{0}\right), t_{0}\right)$ because $z^{*}\left(\cdot, t_{0}\right)$ and $z_{*}\left(\cdot, t_{0}\right)$ are continuous. Then (b) and a comparison result for viscosity solutions of the equation $1-|D z|^{2}=0$ imply that $z^{*}\left(\cdot, t_{0}\right)=d\left(\cdot, t_{0}\right)$ on $\left\{z^{*}<0\right\}$. Similarly, $z_{*}\left(\cdot, t_{0}\right)=d\left(\cdot, t_{0}\right)$ on $\left\{z_{*}>0\right\}$, due to the uniqueness of a viscosity solution for the problem

$$
\left\{\begin{array}{l}
|D z|^{2}-1=0 \text { on }\left\{z_{*}>0\right\} \\
\left.z\right|_{\partial\left\{z_{*}>0\right\}}=0
\end{array}\right.
$$

Finally, by (50) and the restriction on the growth of $z^{*}\left(\cdot, t_{0}\right)$ and $z_{*}\left(\cdot, t_{0}\right)$, we obtain that $z^{*}\left(\cdot, t_{0}\right)=z_{*}\left(\cdot, t_{0}\right)=d\left(\cdot, t_{0}\right)$, that is, $z^{\varepsilon}\left(\cdot, t_{0}\right) \rightarrow d\left(\cdot, t_{0}\right)$ uniformly.

6.2. Asymptotic limit of $E^{\varepsilon}\left[u^{\varepsilon}\right]$. As before, let $\tau$ be the time until which the initial number of interfaces is preserved. We want to prove that $E$ is continuous on $[0, \tau)$ and that $E=E^{0}$ (see Theorem 4 and remarks preceding it for definitions).

REMARK 6. Since by (22) and (24) $\left\{\varepsilon^{\frac{1}{2}} u_{t}^{\varepsilon}\right\}_{\varepsilon>0}$ is bounded in $L^{1}([0, R] \times[0, T])$, Fatou's lemma implies for any $T>0$ that

$$
\int_{0}^{T} \liminf _{\varepsilon \rightarrow 0}\left(\varepsilon \int_{0}^{R}\left(u_{t}^{\varepsilon}\right)^{2} r d r\right) d t \leq M
$$

and thus $\liminf _{\varepsilon \rightarrow 0}\left(\varepsilon \int_{0}^{R}\left(u_{t}^{\varepsilon}\right)^{2} r d r\right)$ is bounded for a.e. $t \in[0, T]$. Denote the set of such $t$ 's by $A$. By the definition of lower limit, for every $t \in A$ there exists a subsequence $\left\{\varepsilon_{n_{k}(t)}\right\}_{k=1}^{\infty}$ such that $\lim _{k \rightarrow \infty}\left(\varepsilon_{n_{k}} \int_{0}^{R}\left(u_{t}^{\varepsilon_{n_{k}}}\right)^{2} r d r\right)<\infty$. Denote the bound on $\left\{\varepsilon_{n_{k}} \int_{0}^{R}\left(u_{t}^{\varepsilon_{n_{k}}}\right)^{2} r d r\right\}_{k=1}^{\infty}$ at time $t$ by $a_{t}$. Observe that since both $A$ and the set of continuity points of $E$ have a full measure, so does their intersection. 
Lemma 3. Let $t \in A$ be a continuity point of $E$. Then, passing to a subsequence $\left\{\varepsilon_{n_{k}(t)}\right\}_{k=1}^{\infty}$ and suppressing the index $n_{k}$ for notational simplicity, we have $\varlimsup_{\varepsilon \rightarrow 0}\left|\lambda^{\varepsilon}(t)\right|$ $\leq C\left(M, a_{t}\right)$. Here $M$ is the uniform in $\varepsilon$ bound on the energy, while $a_{t}$ is specified in the previous remark.

Proof. Multiplying Eq. (32) by $\varepsilon u_{r}^{\varepsilon}$ and integrating it in $r$ from $y$ to $y_{1}$ we have

$$
\varepsilon \int_{y}^{y_{1}} u_{r}^{\varepsilon} u_{t}^{\varepsilon} d r-\left.\left[\varepsilon \frac{\left(u_{r}^{\varepsilon}\right)^{2}}{2}-\frac{W\left(u^{\varepsilon}\right)}{\varepsilon}\right]\right|_{y} ^{y_{1}}-\varepsilon \int_{y}^{y_{1}}\left(u_{r}^{\varepsilon}\right)^{2} \frac{d r}{r}-\left.\lambda^{\varepsilon} h\left(u^{\varepsilon}\right)\right|_{y} ^{y_{1}}=0 .
$$

By Theorem 12 one can choose $y$ and $y_{1} \in(0, R)$, in general depending on $t$, such that

$$
\begin{gathered}
{\left.\left[\varepsilon \frac{\left(u_{r}^{\varepsilon}\right)^{2}}{2}-\frac{W\left(u^{\varepsilon}\right)}{\varepsilon}\right]\right|_{y} ^{y_{1}}=\left.\left[\frac{1}{2 \varepsilon}\left(q^{\prime}\left(z^{\varepsilon} / \varepsilon\right)\right)^{2}\left(\left(z_{r}^{\varepsilon}\right)^{2}-1\right)\right]\right|_{y} ^{y_{1}} \rightarrow 0} \\
h\left(u^{\varepsilon}\left(y_{1}, t\right)\right) \rightarrow \pm \frac{2}{3} \\
h\left(u^{\varepsilon}(y, t)\right) \rightarrow \mp \frac{2}{3}
\end{gathered}
$$

The third term in (51) can be bounded by

$$
\varepsilon \int_{y}^{y_{1}}\left(u_{r}^{\varepsilon}\right)^{2} \frac{d r}{r} \leq \frac{2 M}{y^{2}}
$$

and the first by

$$
\varepsilon \int_{y}^{y_{1}}\left|u_{r}^{\varepsilon} u_{t}^{\varepsilon}\right| d r \leq \frac{1}{y}\left(\varepsilon \int_{y}^{y_{1}}\left(u_{r}^{\varepsilon}\right)^{2} r d r\right)^{\frac{1}{2}}\left(\varepsilon \int_{y}^{y_{1}}\left(u_{t}^{\varepsilon}\right)^{2} r d r\right)^{\frac{1}{2}} \leq \frac{\left(2 M a_{t}\right)^{\frac{1}{2}}}{y} .
$$

The proof follows from (51).

LEMma 4. Let $t$ be as in Lemma 3. Then on a subsequence $\left\{\varepsilon_{n_{k}(t)}\right\}_{k=1}^{\infty}$ one has

$$
\int_{0}^{R}\left[\frac{W\left(u^{\varepsilon}\right)}{\varepsilon}-\varepsilon \frac{\left(u_{r}^{\varepsilon}\right)^{2}}{2}\right] r d r=\frac{1}{2 \varepsilon} \int_{0}^{R}\left(q^{\prime}\left(z^{\varepsilon} / \varepsilon\right)\right)^{2}\left(1-\left(z_{r}^{\varepsilon}\right)^{2}\right) r d r \rightarrow 0 \text { as } \varepsilon \rightarrow 0 .
$$

Proof. (a) Let $r_{i}, i=1, \ldots, N$ be the positions of interfaces at time $t$ (for notational simplicity we omit the variable $t$ ). For any fixed $\delta>0$, set $C_{\delta}:=[0, R] \backslash \bigcup_{i}^{N}\left[r_{i}-\delta, r_{i}+\delta\right]$. Since $z^{\varepsilon}(\cdot, t) \rightarrow d(\cdot, t)$ uniformly, we immediately obtain that

$$
\int_{C_{\delta}}\left[\frac{W\left(u^{\varepsilon}\right)}{\varepsilon}-\varepsilon \frac{\left(u_{r}^{\varepsilon}\right)^{2}}{2}\right] r d r=\frac{1}{2 \varepsilon} \int_{C_{\delta}}\left(q^{\prime}\left(z^{\varepsilon} / \varepsilon\right)\right)^{2}\left(1-\left(z_{r}^{\varepsilon}\right)^{2}\right) r d r \rightarrow 0,
$$

as $\varepsilon \rightarrow 0$ for any $\delta>0$ small.

(b) Now along with $\delta$, fix $i \in\{1, \ldots, N\}$ and integrate (38) in $y$ over $\left[r_{i}-\delta, r_{i}+\delta\right]$, while $y_{1}=r_{i}+\delta$. Then

$$
\begin{aligned}
\varepsilon \int_{r_{i}-\delta}^{r_{i}+\delta} \int_{y}^{r_{i}+\delta} u_{r}^{\varepsilon} u_{t}^{\varepsilon} r d r d y+\frac{\delta}{\varepsilon}\left(r_{i}+\delta\right) & {\left[\frac{W\left(u^{\varepsilon}\left(r_{i}+\delta, t\right)\right)}{\varepsilon}-\varepsilon \frac{\left(u_{r}^{\varepsilon}\left(r_{i}+\delta, t\right)\right)^{2}}{2}\right] } \\
-\int_{r_{i}-\delta}^{r_{i}+\delta}\left(\varepsilon \frac{\left(u_{r}^{\varepsilon}\right)^{2}}{2}-\frac{W\left(u^{\varepsilon}\right)}{\varepsilon}\right) & r d r-\int_{r_{i}-\delta}^{r_{i}+\delta} \int_{y}^{r_{i}+\delta}\left[\varepsilon \frac{\left(u_{r}^{\varepsilon}\right)^{2}}{2}+\frac{W\left(u^{\varepsilon}\right)}{\varepsilon}\right] d r d y \\
& -\lambda^{\varepsilon} \int_{r_{i}-\delta}^{r_{i}+\delta}\left[\left(\left.r h\left(u^{\varepsilon}\right)\right|_{y} ^{r_{i}+\delta}\right)-\int_{y}^{r_{i}+\delta} h\left(u^{\varepsilon}\right) d r\right] d y=0 .
\end{aligned}
$$


Here the terms admit the following bounds:

(1)

$$
\begin{aligned}
\left|\varepsilon \int_{r_{i}-\delta}^{r_{i}+\delta} \int_{y}^{r_{i}+\delta} u_{r}^{\varepsilon} u_{t}^{\varepsilon} r d r d y\right| & \leq \int_{r_{i}-\delta}^{r_{i}+\delta}\left(\varepsilon \int_{y}^{r_{i}+\delta}\left(u_{r}^{\varepsilon}\right)^{2} r d r\right)^{\frac{1}{2}}\left(\varepsilon \int_{y}^{r_{i}+\delta}\left(u_{t}^{\varepsilon}\right)^{2} r d r\right)^{\frac{1}{2}} d y \\
& \leq 2 \delta\left(2 M a_{t}\right)^{\frac{1}{2}}
\end{aligned}
$$

by Hölder's inequality, definition of $A$ and (24);

(2) The term

$$
\begin{aligned}
& \frac{\delta}{\varepsilon}\left(r_{i}+\delta\right)\left[\frac{W\left(u^{\varepsilon}\left(r_{i}+\delta, t\right)\right)}{\varepsilon}-\varepsilon \frac{\left(u_{r}^{\varepsilon}\left(r_{i}+\delta, t\right)\right)^{2}}{2}\right] \\
& \quad=\frac{\delta}{\varepsilon}\left[\left(r_{i}+\delta\right)\left(q^{\prime}\left(z^{\varepsilon}\left(r_{i}+\delta\right) / \varepsilon\right)\right)^{2}\left(1-\left(z_{r}^{\varepsilon}\left(r_{i}+\delta, t\right)\right)^{2}\right)\right]
\end{aligned}
$$

converges to zero when $\varepsilon \rightarrow 0$ (as in (a)):

(3)

$$
\int_{r_{i}-\delta}^{r_{i}+\delta} \int_{y}^{r_{i}+\delta}\left[\varepsilon \frac{\left(u_{r}^{\varepsilon}\right)^{2}}{2}+\frac{W\left(u^{\varepsilon}\right)}{\varepsilon}\right] d r d y \leq \frac{4 M \delta}{r_{i}} \text { by }(24)
$$

(4)

$$
\varlimsup_{\varepsilon \rightarrow 0}\left|\lambda^{\varepsilon} \int_{r_{i}-\delta}^{r_{i}+\delta}\left[\left(\left.r h\left(u^{\varepsilon}\right)\right|_{y} ^{r_{i}+\delta}\right)-\int_{y}^{r_{i}+\delta} h\left(u^{\varepsilon}\right) d r\right] d y\right| \leq \frac{8}{3} C\left(M, a_{t}\right) R \delta
$$

by definition of $h\left(u^{\varepsilon}\right)$ and the previous lemma.

Thus

$$
\varlimsup_{\varepsilon \rightarrow 0}\left[\frac{1}{2 \varepsilon} \int_{r_{i}-\delta}^{r_{i}+\delta}\left(q^{\prime}\left(z^{\varepsilon} / \varepsilon\right)\right)^{2}\left(1-\left(z_{r}^{\varepsilon}\right)^{2}\right) r d r\right] \leq C\left(M, a_{t}, r_{i}\right) \delta .
$$

For a given $\delta>0$ similar estimates hold for all $i=1, \ldots, N$.

(c) From (a) and (b)

$$
\varlimsup_{\varepsilon \rightarrow 0}\left[\frac{1}{2 \varepsilon} \int_{0}^{R}\left(q^{\prime}\left(z^{\varepsilon} / \varepsilon\right)\right)^{2}\left(1-\left(z_{r}^{\varepsilon}\right)^{2}\right) r d r\right] \leq C\left(M, a_{t}, \min _{1 \leq i \leq N} r_{i}\right) \delta
$$

for any $\delta>0$. Letting $\delta \rightarrow 0$ we deduce that

$$
\lim _{\varepsilon \rightarrow 0} \frac{1}{2 \varepsilon} \int_{0}^{R}\left(q^{\prime}\left(z^{\varepsilon} / \varepsilon\right)\right)^{2}\left(1-\left(z_{r}^{\varepsilon}\right)^{2}\right) r d r=0
$$

Lemma 5. Let $t$ and $r_{i}, i=1, \ldots, N$ be the same as in the previous lemma. Then on a subsequence $\left\{\varepsilon_{n_{k}(t)}\right\}_{k=1}^{\infty}$ we have (suppressing the index $n_{k}$ for notational simplicity)

$$
E^{\varepsilon}\left[u^{\varepsilon}\right](t) \rightarrow \frac{8 \pi}{3} \sum_{i=1}^{N} r_{i}(t)
$$


Proof. Fix $\delta>0$ small. Then by (20) we have

$$
\begin{aligned}
E^{\varepsilon}\left[u^{\varepsilon}\right](t)= & \frac{\pi}{\varepsilon} \int_{0}^{R}\left(q^{\prime}\right)^{2}\left(1+\left(z_{r}^{\varepsilon}\right)^{2}\right) r d r \leq \frac{\pi}{\varepsilon} \int_{0}^{R}\left(q^{\prime}\right)^{2}\left(1-\left(z_{r}^{\varepsilon}\right)^{2}\right) r d r \\
& +\frac{2 \pi}{\varepsilon} \int_{C_{\delta}}\left(q^{\prime}\right)^{2}\left|z_{r}^{\varepsilon}\right| r d r+\sum_{i=1}^{N}\left[\frac{2 \pi}{\varepsilon} \int_{r_{i}-\delta}^{r_{i}+\delta}\left(q^{\prime}\right)^{2}\left|z_{r}^{\varepsilon}\right| r d r\right],
\end{aligned}
$$

where $C_{\delta}$ is as in Lemma 4 . Observe that the first and the second integrals on the righthand side converge to zero by the previous lemma and due to the uniform convergence of $z^{\varepsilon}(\cdot, t) \rightarrow d(\cdot, t)$. Also by Theorem $12, z_{r}^{\varepsilon}$ has a constant sign on each of $\left[r_{i}-\delta, r_{i}+\delta\right]$ if $\varepsilon>0$ is small. Then by computing the integral in the third term by parts and letting $\varepsilon \rightarrow 0$ we obtain

$$
\lim _{\varepsilon \rightarrow 0} \sum_{i=1}^{N}\left[\frac{2 \pi}{\varepsilon} \int_{r_{i}-\delta}^{r_{i}+\delta}\left(q^{\prime}\right)^{2}\left|z_{r}^{\varepsilon}\right| r d r\right]=\frac{8 \pi}{3} \sum_{i=1}^{N} r_{i}(t)
$$

which, together with Theorem 4, implies (53).

THEOREM 13. $E$ is continuous on $[0, \tau)$ and

$$
E^{\varepsilon}\left[u^{\varepsilon}\right](t) \rightarrow \frac{8 \pi}{3} \sum_{i=1}^{N} r_{i}(t)
$$

for every $t \in[0, \tau)$.

Proof. Since for every continuity point $t$ of $E$ in $A$, we have that $E^{\varepsilon}\left[u^{\varepsilon}\right](t) \rightarrow E(t)$ on the whole sequence, $E(t)=\frac{8 \pi}{3} \sum_{i=1}^{N} r_{i}(t)$ by the previous lemma. By Remark 6 , the set of points for which Lemma 5 holds is dense in $[0, \tau)$. Then, since the positions of the interfaces are continuous in time (see Theorem 3 ) and since $E$ is monotone, we conclude that $E$ is continuous on $[0, \tau)$ and thus $E=E^{0}[v]$ on $[0, \tau)$.

Remark 7. Since $E$ is continuous on $[0, \tau)$, using Theorem 12 we obtain that $z^{\varepsilon} \rightarrow d$ locally uniformly on $[0, R] \times[0, \tau)$.

THEOREM 14. $E^{\varepsilon}\left[u^{\varepsilon}\right] \rightarrow E$ uniformly on compact subsets of $(0, \tau)$ as $\varepsilon \rightarrow 0$.

Proof. More generally, suppose that there is a family $\left\{f_{n}\right\}_{n \in \mathbf{N}}$ of uniformly bounded monotone continuous functions on an interval $(a, b)$, converging to a monotone continuous function $f$ in $L^{1}((a, b))$. For all $\delta>0$ and $n \in \mathbf{N}$ set $\Delta_{\delta}^{n}:=\left\{t:\left|f(t)-f_{n}(t)\right|>\delta\right\}$. Then meas $\left(\Delta_{\delta}^{n}\right) \rightarrow 0$ as $n \rightarrow \infty$ for any $\delta>0$, where meas $(A)$ is the Lebesgue measure of the set $A$. Choose $\left[t_{1}, t_{2}\right] \subset(a, b)$ and suppose that $f_{n}$ does not converge to $f$ uniformly on $\left[t_{1}, t_{2}\right]$. Then there exists $\delta>0$ and a subsequence $\left\{n_{k}\right\}_{k \in \mathbf{N}}$ such that $\left[t_{1}, t_{2}\right] \cap \Delta_{\delta}^{n_{k}} \neq \varnothing$ for all $k \in \mathbf{N}$. For every $k \in \mathbf{N}$ select $s_{n_{k}} \in\left[t_{1}, t_{2}\right] \cap \Delta_{\delta}^{n_{k}}$, and passing yet to another subsequence we have that $s_{n_{k}} \rightarrow s \in\left[t_{1}, t_{2}\right]$ as $k \rightarrow \infty$.

In what follows we will omit the index $k$ for notational simplicity. Suppose that $\left\{f_{n}\right\}_{n \in \mathbf{N}}$ and $f$ are monotone decreasing. Since $f$ is continuous on $(a, b)$, there exists $h>0$ such that $|f(s+h)-f(s-h)|<\delta / 4$. Since meas $\left(\Delta_{\delta / 2}^{n}\right) \rightarrow 0$, we can find $l \in \mathbf{N}$ such 
that $s_{l} \in(s-h, s+h)$ and there exist $p_{1} \in\left(s-h, s_{l}\right) \cap \Delta_{\delta / 2}^{l}$ and $p_{2} \in\left(s_{l}, s+h\right) \cap \Delta_{\delta / 2}^{l}$. Assume at first that $f\left(s_{l}\right)-f_{l}\left(s_{l}\right) \geq 0$; then $f\left(s_{l}\right)-\delta \geq f_{l}\left(s_{l}\right)$ and also

$$
f\left(s_{l}\right)=f\left(p_{2}\right)+\left(f\left(s_{l}\right)-f\left(p_{2}\right)\right) \leq f\left(p_{2}\right)+\delta / 4 \leq f_{l}\left(p_{2}\right)+3 \delta / 4
$$

then

$$
f_{l}\left(p_{2}\right)-\delta / 4 \geq f_{l}\left(s_{l}\right)
$$

which is a contradiction since $f_{l}$ is monotone decreasing. If $f\left(s_{l}\right)-f_{l}\left(s_{l}\right) \leq 0$ then the same procedure works with $p_{1}$ replaced by $p_{2}$. The case when $\left\{f_{n}\right\}_{n \in \mathbb{N}}$ and $f$ are monotone increasing can be handled in a similar manner.

THEOREM 15. $E$ is locally Lipschitz continuous on $(0, \tau)$.

Proof. As in the proof of (25) (see [6]), we have by Hölder's inequality that for any $t_{1}, t_{2} \in[0, T]$

$$
\begin{aligned}
\int_{t_{1}}^{t_{2}} \int_{\Omega}\left|h_{t}^{\varepsilon}(x, t)\right| d x d t & \leq\left(\int_{t_{1}}^{t_{2}} \int_{\Omega} \varepsilon\left(u_{t}^{\varepsilon}(x, t)\right)^{2} d x d t\right)^{1 / 2}\left(\int_{t_{1}}^{t_{2}} \int_{\Omega} \frac{\left(W\left(u^{\varepsilon}\right)\right)^{2}}{2 \varepsilon} d x d t\right)^{1 / 2} \\
& \leq C\left(t_{2}-t_{1}\right)^{1 / 2}\left(E^{\varepsilon}\left[u^{\varepsilon}\right]\left(t_{1}\right)-E^{\varepsilon}\left[u^{\varepsilon}\right]\left(t_{2}\right)\right)^{1 / 2}
\end{aligned}
$$

where $h^{\varepsilon}(x, t)=h\left(u^{\varepsilon}(x, t)\right)$ and $C$ is independent of $\varepsilon$. At the same time

$$
\int_{t_{1}}^{t_{2}} \int_{\Omega}\left|h_{t}^{\varepsilon}(x, t)\right| d x d t \geq \int_{\Omega}\left|h^{\varepsilon}\left(x, t_{2}\right)-h^{\varepsilon}\left(x, t_{1}\right)\right| d x .
$$

Then, by the Dominated Convergence Theorem, we have for any $t_{1}, t_{2} \in(0, \tau)$ that as $\varepsilon \rightarrow 0$

$$
\int_{\Omega}\left|h\left(x, t_{2}\right)-h\left(x, t_{1}\right)\right| d x \leq C\left(t_{2}-t_{1}\right)^{1 / 2}\left(E\left(t_{1}\right)-E\left(t_{2}\right)\right)^{1 / 2} .
$$

Here $h(x, t)=h(v(x, t))$. Since $v$ takes values +1 or -1 a.e.,

$$
\left|h\left(x, t_{2}\right)-h\left(x, t_{1}\right)\right|=\frac{2}{3}\left|v\left(x, t_{2}\right)-v\left(x, t_{1}\right)\right| \text { a.e. }
$$

Choose a compact set $A \in(0, \tau)$. Since $v(x, t)$ is known explicitly on $(0, \tau)$, by Theorem 13

$$
\begin{aligned}
\int_{\Omega} \frac{2}{3}\left|v\left(x, t_{2}\right)-v\left(x, t_{1}\right)\right| d x & =\frac{4 \pi}{3} \sum_{i=1}^{N}\left|r_{i}^{2}\left(t_{2}\right)-r_{i}^{2}\left(t_{1}\right)\right| \\
& \geq \frac{8 \pi}{3} \min _{t \in A} r_{1}(t) \sum_{i=1}^{N}\left|r_{i}\left(t_{2}\right)-r_{i}\left(t_{1}\right)\right| \\
& \geq C_{1}\left(E\left(t_{1}\right)-E\left(t_{2}\right)\right),
\end{aligned}
$$

for any $t_{1}, t_{2} \in A$, where $C_{1}$ depends only on $A$. Therefore, for any $t_{1}, t_{2} \in A$

$$
E\left(t_{1}\right)-E\left(t_{2}\right) \leq C\left(t_{2}-t_{1}\right)^{1 / 2}\left(E\left(t_{1}\right)-E\left(t_{2}\right)\right)^{1 / 2} \text {. }
$$


Dividing both sides by $\left(E\left(t_{1}\right)-E\left(t_{2}\right)\right)^{1 / 2}$, we conclude that $E$ is locally Lipschitz continuous on $(0, \tau)$, with Lipschitz constant depending on the energy bound $M$ and the set A.

This result is sharp in $\mathbf{R}^{2}$ since, as we will see, each of the interfaces moves by its curvature. Then, if at least one of the interfaces shrinks to zero at a time $t$, the limiting energy, which depends linearly on a position of every interface, cannot be differentiable at $t$.

REMARK 8. By slightly changing the proof of the previous theorem, one can show that the $r_{i}$ are locally Lipschitz continuous on $(0, \tau)$ for all $i=1, \ldots, N$.

REMARK 9. Multiplying (51) by $\varepsilon$ and choosing $y_{1}$ appropriately we have that for any continuity point $t>0$ of the limiting energy $E$

$$
\left(q^{\prime}\left(z^{\varepsilon} / \varepsilon\right)\right)^{2}\left(\left(z_{r}^{\varepsilon}\right)^{2}-1\right) \rightarrow 0
$$

uniformly on $[0, R]$. Then, by Remark 7 ,

$$
\left(q^{\prime}\left(z^{\varepsilon} / \varepsilon\right)\right)^{2}\left(\left(z_{r}^{\varepsilon}\right)^{2}-1\right) \rightarrow 0
$$

uniformly on $[0, R] \times\left[t_{1}, t_{2}\right]$.

REMARK 10. Choose a sequence $\left\{h_{n}\right\}_{n \in \mathbf{N}} \subset \mathbf{R}$ such that $h_{n} \rightarrow 0$ as $n \rightarrow \infty$. Integrating Eq. (51) in $t$ for the appropriate $y$ and $y_{1}$, using the previous remark and passing, if necessary, to a subsequence we obtain that the averages

$$
\lambda_{h_{n}}^{\varepsilon}(t):=\frac{2}{h_{n}} \int_{t}^{t+h_{n}} \lambda^{\varepsilon}(s) d s
$$

converge uniformly as $\varepsilon \rightarrow 0$ to a limit $\lambda_{h_{n}}$ for each $n \in \mathbf{N}$. Moreover, by Theorem 15, the equation $\left\{\lambda_{h_{n}}\right\}_{n \in \mathbf{N}}$ is locally uniformly bounded on $(0, \tau)$.

6.3. Convergence of reaction-diffusion equations to a nonlocal mean curvature flow. Our next goal is to determine the dynamics of the moving interfaces on an interval $[0, \tau)$. We will use the following strategy. First, as in [3], we are going to show that the distance function $d$ satisfies certain differential inequalities in a viscosity sense. Then using these inequalities we will determine for every interface that its position solves a certain ODE (again, in a viscosity sense). Finally, we will prove that if the initial positions of interfaces are given, the uniqueness of the classical solution of the latter system of ODE's implies that interfaces solve this system of ODE's in a classical sense.

We can prove the following refinement of Remark 10.

Lemma 6. Let $\lambda_{h}^{\varepsilon}$ be as in (56). There exist $\lambda_{h} \in C([0, \tau-\gamma])$ and $\lambda \in C([0, \tau-\gamma])$ such that

a)

$$
\lambda_{h}^{\varepsilon} \rightarrow \lambda_{h} \text { uniformly on }[0, \tau-\gamma] \text { as } \varepsilon \rightarrow 0
$$

b)

$$
\lambda_{h} \rightarrow \lambda \text { uniformly on }[0, \tau-\gamma] \text { as } h \rightarrow 0 \text {. }
$$


Moreover,

$$
\lambda(t)=\frac{a_{N}(t)}{\sum_{i=1}^{N} r_{i}(t)}
$$

Here $N$ is the number of interfaces, and $a_{N}$ is a function depending on the geometry of the problem,

$$
a_{N}(t)=\frac{v(R, t)-v(0, t)}{2}
$$

REMARK 11. This particular expression for $\lambda$ is valid only in two dimensions.

Proof. Multiply Eq. (32) by $\varepsilon r\left|u_{r}^{\varepsilon}\right|$ and integrate by parts over $[0, R]$. Then for $\varepsilon>0$ small we obtain

$$
\varepsilon \int_{0}^{R}\left|u_{r}^{\varepsilon}\right| u_{t}^{\varepsilon} r d r-\frac{\varepsilon}{2} \int_{0}^{R}\left|u_{r}^{\varepsilon}\right| u_{r}^{\varepsilon} d r+\frac{1}{\varepsilon} \int_{0}^{R} W^{\prime}\left(u^{\varepsilon}\right)\left|u_{r}^{\varepsilon}\right| r d r-\lambda^{\varepsilon} \int_{0}^{R} h^{\prime}\left(u^{\varepsilon}\right)\left|u_{r}^{\varepsilon}\right| r d r=0
$$

Since $z^{\varepsilon} \rightarrow d$ uniformly on $[0, R] \times[0, \tau-\gamma]$ and due to our condition on the number of zeroes of $z_{r}^{\varepsilon}$, we immediately obtain that zeroes of $z_{r}^{\varepsilon}$ are uniformly at a distance of order 1 away from the interfaces if $\varepsilon>0$ is small enough. It is also easy to observe that the coefficient of $\lambda^{\varepsilon}$,

$$
B^{\varepsilon}:=-\int_{0}^{R} h^{\prime}\left(u^{\varepsilon}\right)\left|u_{r}^{\varepsilon}\right| r d r=\frac{1}{2 \pi} \int_{\Omega}\left|D h\left(u^{\varepsilon}\right)\right| d x
$$

converges uniformly as $\varepsilon>0$ to $\frac{4}{3} \sum_{i=1}^{N} r_{i}$, since $z^{\varepsilon} \rightarrow d$ uniformly on $[0, R] \times[0, \tau-\gamma]$. Since $r_{i}$ are uniformly bounded away from zero on the interval $[0, \tau-\gamma]$ for every $i=$ $1, \ldots, N$, we can divide $(58)$ by $B_{\varepsilon}$ and integrate the result by parts over $(t, t+h)$ to obtain

$$
\begin{array}{r}
-\varepsilon \int_{t}^{t+h} B_{\varepsilon}^{-1}(s) \int_{0}^{R}\left|u_{r}^{\varepsilon}\right| u_{t}^{\varepsilon} r d r d s+\int_{t}^{t+h} B_{\varepsilon}^{-1}(s)\left(\sum_{i \in A_{+}}\left\{\int_{r_{i}-\delta}^{r_{i}+\delta}\left(\frac{\varepsilon}{2}\left(u_{r}^{\varepsilon}\right)^{2}+\frac{1}{\varepsilon} W\left(u^{\varepsilon}\right)\right) d r\right\}\right. \\
\left.-\sum_{i \in A_{-}}\left\{\int_{r_{i}-\delta}^{r_{i}+\delta}\left(\frac{\varepsilon}{2}\left(u_{r}^{\varepsilon}\right)^{2}+\frac{1}{\varepsilon} W\left(u^{\varepsilon}\right)\right) d r\right\}\right) d s+o(1)=\int_{t}^{t+h} \lambda^{\varepsilon}(s) d s
\end{array}
$$

for sufficiently small, positive, $\varepsilon, h$, and $\delta$. Here $o(1)$ denotes the terms uniformly convergent to zero on $[0, t-\gamma]$ as $\varepsilon \rightarrow 0$, while $A_{+}$is a set of indices of the interfaces with $d_{r}>0$, and $A_{-}$is a set of indices of the interfaces with $d_{r}<0$. Note that

$$
\frac{1}{2 \varepsilon} \int_{t}^{t+h} \int_{0}^{R}\left(q^{\prime}\left(z^{\varepsilon} / \varepsilon\right)\right)^{2}\left(1-\left|z_{r}^{\varepsilon}\right|\right)^{2} r d r d s \rightarrow 0 \text { as } \varepsilon \rightarrow 0
$$

uniformly on $[0, t-\gamma]$. From (13) and since $q^{\prime}=1-q^{2}=g(q)$ we have

$$
0=\frac{d}{d t} \int_{0}^{R} h\left(u^{\varepsilon}\right) r d r=\int_{0}^{R} g\left(u^{\varepsilon}\right) u_{t}^{\varepsilon} r d r=\frac{1}{\varepsilon} \int_{0}^{R}\left(q^{\prime}\left(z^{\varepsilon} / \varepsilon\right)\right)^{2} z_{t}^{\varepsilon} r d r .
$$


Then by (60), Hölder's inequality, and the remark above,

$$
\begin{gathered}
\left|\varepsilon \int_{t}^{t+h} B_{\varepsilon}^{-1}(s) \int_{0}^{R}\right| u_{r}^{\varepsilon}\left|u_{t}^{\varepsilon} r d r d s\right|=\left|\frac{1}{\varepsilon} \int_{t}^{t+h} B_{\varepsilon}^{-1}(s) \int_{0}^{R}\left(q^{\prime}\left(z^{\varepsilon} / \varepsilon\right)\right)^{2}\right| z_{r}^{\varepsilon}\left|z_{t}^{\varepsilon} r d r d s\right| \\
\quad=\left|\frac{1}{\varepsilon} \int_{t}^{t+h} B_{\varepsilon}^{-1}(s) \int_{0}^{R}\left(q^{\prime}\left(z^{\varepsilon} / \varepsilon\right)\right)^{2}\left(1-\left|z_{r}^{\varepsilon}\right|\right) z_{t}^{\varepsilon} r d r d s\right| \\
\leq \sup _{s \in[t, t+h]} B_{\varepsilon}^{-1}(s)\left(\int_{t}^{t+h} \int_{0}^{R}\left(q^{\prime}\left(\frac{z^{\varepsilon}}{\varepsilon}\right)\right)^{2} \frac{\left(1-\left|z_{r}^{\varepsilon}\right|\right)^{2}}{\varepsilon} r d r d s\right)^{\frac{1}{2}} \\
\quad \times\left(\varepsilon \int_{t}^{t+h} \int_{0}^{R}\left(u_{t}^{\varepsilon}\right)^{2} r d r d s\right)^{\frac{1}{2}} \\
\rightarrow 0,
\end{gathered}
$$

as $\varepsilon \rightarrow 0$ uniformly on $[0, t-\gamma]$. It is easy to show that the remaining term in (59) converges uniformly to

$$
\int_{t}^{t+h} \frac{a_{N}(s)}{\sum_{i=1}^{N} r_{i}(s)} d s
$$

where $a_{N}$ defined in (57) is either $+1,-1$, or 0 depending on the relative number of the interfaces from $A_{+}$and $A_{-}$. Thus, for any $h>0$ small,

$$
\lambda_{h}^{\varepsilon} \rightarrow \lambda_{h}=\frac{1}{h} \int_{t}^{t+h} \frac{a_{N}(s)}{\sum_{i=1}^{N} r_{i}(s)} d s
$$

uniformly on $[0, t-\gamma]$ as $\varepsilon \rightarrow 0$. Now since $a_{N}(\cdot) / \sum_{i=1}^{N} r_{i}(\cdot)$ is continuous on a compact set $[0, t-\gamma]$,

$$
\lambda_{h} \rightarrow \frac{a_{N}(\cdot)}{\sum_{i=1}^{N} r_{i}(\cdot)}
$$

uniformly on $[0, t-\gamma]$ as $h \rightarrow 0$.

We remind the reader that this formula for $\lambda$ holds only in $\mathbf{R}^{2}$. For higher dimensions $\lambda$ will be determined by a different formula.

Theorem 16. Assume that $\phi \in C^{\infty}([0, R] \times[0, \tau))$ is such that $d-\phi$ has a maximum at $\left(r_{0}, t_{0}\right) \in(0, R) \times(0, t)$ and $d\left(r_{0}, t_{0}\right)<0$. Then

$$
\phi_{t}-\Delta \phi+\lambda \leq 0 \text { at }\left(r_{0}, t_{0}\right)
$$

where $\lambda(t)$ is as in Lemma 6 .

REMARK 12. Without loss of generality we can assume that the maximum of $d-\phi$ is strict. Indeed, this can be achieved by replacing $\phi$ by

$$
\phi_{\alpha \beta}(r, t)=\phi(r, t) \frac{\left(r-r_{0}\right)^{4}}{\alpha}+\frac{\left(t-t_{0}\right)^{2}}{\beta}
$$

and choosing $\alpha$ and $\beta$ to be small enough. 
Proof. The proof is based on a viscosity argument of [3]. First fix $\gamma>0$ and let $\gamma>h>0$. Suppose $\Omega_{\gamma}:=[0, R] \times[0, \tau-\gamma]$. Set

$$
\begin{aligned}
z_{h}^{\varepsilon} & =\frac{1}{h} \int_{t}^{t+h} z^{\varepsilon}(x, s) d s \\
d_{h} & =\frac{1}{h} \int_{t}^{t+h} d(x, s) d s \\
\phi_{h} & =\frac{1}{h} \int_{t}^{t+h} \phi(x, s) d s .
\end{aligned}
$$

Since $z^{\varepsilon} \rightarrow d$ uniformly on $[0, R] \times[0, \tau)$ we have that

(a) $z_{h}^{\varepsilon} \rightarrow d_{h}$ uniformly as $\varepsilon \rightarrow 0$ on $\Omega_{\gamma}$;

(b) $d_{h} \rightarrow d, \frac{\partial^{i}}{\partial r^{i}} \phi_{h} \rightarrow \frac{\partial^{i}}{\partial r^{i}} \phi, \frac{\partial}{\partial t} \phi_{h} \rightarrow \frac{\partial}{\partial t} \phi$ for $i=1,2$, uniformly on $\Omega_{\gamma}$ as $h \rightarrow 0$ since both $d$ and $\phi$ together with its derivatives are continuous on the compact set $[0, R] \times[0, \tau-\gamma+h]$.

Since $d_{h}-\phi_{h}$ converges to $d-\phi$ uniformly for $h>0$ small, $d_{h}-\phi_{h}$ achieves its local maximum in a small neighborhood $A_{h}:=\left[r_{0}-a_{h}, r_{0}+a_{h}\right] \times\left[t_{0}-b_{h}, t_{0}+b_{h}\right]$ of $\left(r_{0}, t_{0}\right)$ such that $a_{h}, b_{h} \downarrow 0$ as $h \rightarrow 0$. Similarly, since $z_{h}^{\varepsilon} \rightarrow d_{h}$ uniformly, for $\varepsilon>0$ small, $z_{h}^{\varepsilon}-\phi_{h}$ has a maximum in a small neighborhood $A_{\varepsilon h}:=\left[r_{0}-a_{\varepsilon h}, r_{0}+a_{\varepsilon h}\right] \times\left[t_{0}-b_{\varepsilon h}, t_{0}+b_{\varepsilon h}\right]$ of $\left(r_{0}, t_{0}\right)$ and such that $a_{\varepsilon h} \downarrow a_{h}, b_{\varepsilon h} \downarrow b_{h}$ as $\varepsilon \rightarrow 0$. Observe also that if $h$ and $\varepsilon$ are given, $\left|A_{\varepsilon h}\right|$ can be made arbitrarily small by choosing $\alpha$ and $\beta$ small enough, where $\alpha$ and $\beta$ are as in Remark 12. This implies that we can find $h_{1}, \varepsilon_{1}>0$ such that for any $\varepsilon_{1}>\varepsilon>0$ and $h_{1}>h>0$,

$$
z^{\varepsilon}(r, t)<0 \text { if }(r, t) \in\left[r_{0}-a_{\varepsilon h}, r_{0}+a_{\varepsilon h}\right] \times\left[t_{0}-b_{\varepsilon h}, t_{0}+b_{\varepsilon h}+h\right] .
$$

By integrating Eq. (17) in $t$ we obtain

$$
z_{h t}^{\varepsilon}-\Delta z_{h}^{\varepsilon}+\frac{2}{h} \int_{t}^{t+h} \lambda^{\varepsilon}(s) d s+\frac{2}{\varepsilon h} \int_{t}^{t+h} q^{\varepsilon}\left(\frac{z^{\varepsilon}(r, s)}{\varepsilon}\right)\left(\left(z_{r}^{\varepsilon}(r, s)\right)^{2}-1\right) d s=0
$$

on $\Omega_{\gamma}$. Then by the definition of $q^{\varepsilon}$ and Theorem 7 , the last integral is positive in $A_{\varepsilon h}$. Fix any $\left(r_{\varepsilon h}, t_{\varepsilon h}\right) \in A_{\varepsilon h}$ where $z_{h}^{\varepsilon}-\phi_{h}$ has a local maximum; by (62) we have

$$
\phi_{h t}-\Delta \phi_{h}+\lambda_{h}^{\varepsilon} \leq 0 \text { at }\left(r_{\varepsilon h}, t_{\varepsilon h}\right)
$$

where $\lambda_{h}^{\varepsilon}(t)$ is as in (56). By Lemma $6, \lambda_{h}^{\varepsilon} \rightarrow \lambda_{h}$ uniformly as $\varepsilon \rightarrow 0$ and, in turn $\lambda_{h} \rightarrow \lambda$ uniformly as $h \rightarrow 0$. Let $\varepsilon \rightarrow 0$. Passing to a subsequence, we obtain that

$$
\left(r_{\varepsilon h}, t_{\varepsilon h}\right) \rightarrow\left(r_{h}, t_{h}\right) \in \bar{A}_{h}
$$

and that

$$
\phi_{h t}-\Delta \phi_{h}+\lambda_{h} \leq 0 \text { at }\left(r_{h}, t_{h}\right) .
$$

Letting now $h \rightarrow 0$ and following the same procedure as for $\varepsilon$, we obtain

$$
\phi_{t}-\Delta \phi+\lambda \leq 0 \text { at }\left(r_{0}, t_{0}\right)
$$


Suppose now that $\phi \in C^{\infty}([0, R] \times[0, \tau))$ is such that $d-\phi$ has a maximum at $\left(r_{i}\left(t_{0}\right), t_{0}\right)$ and the $i$ th interface is such that $d_{r}\left(t_{0}, r_{i}\left(t_{0}\right)\right)>0$. Since in the neighborhood of $\left(r_{i}\left(t_{0}\right), t_{0}\right)$ the distance function $d(r, t) \equiv r-r_{i}(t)$ and $r_{i}$ is continuous on $[0, \tau-\gamma]$ we have that, for $d>0$ small,

$$
\phi_{\delta}:=\phi(r+\delta, t)+\delta \in C^{\infty}([0, R] \times[0, \tau))
$$

is such that $d-\phi_{\delta}$ has a maximum at $\left(r_{i}\left(t_{0}\right)-\delta, t_{0}\right)$. Then by $(61)$

$$
\phi_{\delta t}-\Delta \phi_{\delta}+\lambda \leq 0 \text { at }\left(r_{i}\left(t_{0}\right)-\delta, t_{0}\right)
$$

and thus

$$
\phi_{t}-\Delta \phi+\lambda \leq 0 \text { at }\left(r_{i}\left(t_{0}\right), t_{0}\right) .
$$

Therefore, $d$ is a viscosity subsolution of

$$
z_{t}-\Delta z+\lambda=0 \text { at }\left(r_{i}\left(t_{0}\right), t_{0}\right) .
$$

Similarly, we can show that $d$ is also a viscosity supersolution and thus a viscosity solution of $(64)$ at $\left(r_{i}\left(t_{0}\right), t_{0}\right)$ for any $t_{0} \in[0, \tau-\gamma]$. Since $d$ is known explicitly for any $t \in[0, \tau)$ and constructing a test function in a way similar to that of Theorem 12 , we find that $r_{i}$ is a viscosity solution of

$$
\dot{r}_{i}+\frac{1}{r_{i}}-\frac{a_{N}}{\sum_{i=1}^{N} r_{i}}=0
$$

The same procedure for the interfaces with $d_{r}\left(t_{0}, r_{i}\left(t_{0}\right)\right)<0$ shows that $r_{i}$ satisfies

$$
\dot{r}_{i}+\frac{1}{r_{i}}+\frac{a_{N}}{\sum_{i=1}^{N} r_{i}}=0
$$

in a viscosity sense. Given the initial locations of the interfaces, the system of ODE's (65) and (66) has a unique classical solution. Moreover, since for a given $\lambda$ each of the equations (65) and (66) has a unique viscosity solution, $\bar{r}=\left(r_{1}, \ldots, r_{N}\right)$ is a classical solution of $(65-66)$ on $[0, \tau-\gamma]$ and, since $\gamma$ is arbitrary, on $[0, \tau)$. The mass preservation property of the limiting flow follows directly from (65) and (66), or by considering an asymptotic limit of (13). Once again, the formula for $\lambda$ in higher dimensions will be different from (63) and (64).

Any subsequence of our original sequence in $\varepsilon$ contains a subsequence such that $u^{\varepsilon}$ converges in $L^{1}([0, R] \times[0, \tau))$ to a limit $v$ (that may, in general, depend on the subsequence) satisfying:

(a) $v(x, t) \in\{-1,1\}$ a.e. on $[0, R] \times[0, \tau)$,

(b) $\lim _{t \rightarrow 0} v(x, t)=\phi(x)$ a.e. on $[0, R]$,

with interfaces moving according to (65-66). However, since a function with these properties is unique in $L^{1}([0, R] \times[0, \tau))$, then $v$ is the same for all subsequences. Therefore, $v$ is an asymptotic limit of $u^{\varepsilon}$ on the whole sequence.

Observe that if $N$ is even, then $\lambda$ is zero and therefore interfaces simply move by their mean curvature. Hence our solution exists at least up to the time when the innermost interface shrinks to zero. No interfaces can collide with each other in this case. However, the latter may occur if $N$ is odd. We consider this situation in the next section. 


\section{Asymptotic behavior of reaction-diffusion equations.}

7.1. General initial data. "Ghost" interfaces. Let $\tau$ be the first time when any two interfaces of the opposite sign meet. Then after the collision these interfaces might disappear, repulse, or continue to move together. The behavior of the coupled pair of interfaces that form in the latter case cannot be immediately analyzed using the procedure that we employed in the previous sections. It is not clear, for example, whether such pairs will move continuously in time. Indeed, the coupled pair of interfaces corresponds to a discontinuity of the limiting solution $v$ in the region, where this solution is constant. Since a position of the discontinuity can be chosen arbitrarily for any $t \in[\tau, T]$, without changing $v \in L^{1}(\Omega \times[0, T])$, we can no longer use (25) to deduce the continuous evolution of the coupled pair of interfaces. Thus, in order to continue our analysis past the time $\tau$, we have to generalize our results to a wider class of initial data.

From now on suppose that (31) is no longer valid. As we have already mentioned in the previous paragraph, no information on the dynamics of coupled pairs of interfaces can be obtained from the $L^{1}$-limit $v$ of solutions $u^{\varepsilon}$ of (12). Hence, we begin this section by studying the pointwise behavior of $u^{\varepsilon}(\cdot, t)$ on $[0, R]$ as $\varepsilon \rightarrow 0$.

Let

$$
\begin{aligned}
N_{t} & :=\operatorname{int} \operatorname{cl}\left\{r \in(0, R): u^{\varepsilon}(r, t) \rightarrow-1\right\}, \\
P_{t} & :=\operatorname{int} \operatorname{cl}\left\{r \in(0, R): u^{\varepsilon}(r, t) \rightarrow+1\right\},
\end{aligned}
$$

where int $A$ is an interior of the set $A$ and $\operatorname{cl} A$ is its closure. $N_{t}$ and $P_{t}$ are clearly nonempty for all $t>0$, since neither $v(r, 0)=+1$ a.e. $r \in(0, R)$ nor $v(r, 0)=-1$ a.e. $r \in(0, R)$ by our assumptions on the initial data. For every $t \geq 0$ and $r \in[0, R]$ define

$$
u^{*}(r, t)=\limsup _{\substack{\varepsilon \rightarrow 0 \\ s \rightarrow r}} u^{\varepsilon}(s, t)
$$

and

$$
u_{*}(r, t)=\liminf _{\substack{\varepsilon \rightarrow 0 \\ s \rightarrow r}} u^{\varepsilon}(s, t)
$$

Then $u^{*}(\cdot, t)$ is an upper semicontinuous and $u_{*}(\cdot, t)$ is a lower semicontinuous function on $[0, R]$ for any $t \geq 0$. For each $0<\delta<1$ set

$$
\begin{aligned}
N_{t}^{\delta} & :=\left\{r \in N_{t}, u^{*}(r, t) \geq-\delta\right\}, \\
P_{t}^{\delta} & :=\left\{r \in P_{t}, u_{*}(r, t) \leq \delta\right\} .
\end{aligned}
$$

REMARK 13. Fix $t_{0}>0$ and an arbitrary $\rho \in(0, R)$. Suppose that $0<\delta<1$ is given. Since the energy is bounded, passing to a subsequence, one can assume that there are finitely many points in both $N_{t_{0}}^{\delta} \cap[\rho, R]$ and $P_{t_{0}}^{\delta} \cap[\rho, R]$. Indeed, if $r \in N_{t_{0}}^{\delta}$ then by the definition of $N_{t_{0}}^{\delta}$ there exist subsequences $\left\{\varepsilon_{j}\right\}_{j \in \mathbf{N}}$ and $\left\{s_{j}\right\}_{j \in \mathbf{N}}$ such that $\varepsilon_{j} \rightarrow 0$ and $s_{j} \rightarrow r$. At the same time, $\lim _{j \rightarrow \infty} u^{\varepsilon_{j}}\left(s_{j}, t_{0}\right) \geq-\delta$ while $u^{\varepsilon_{j}}\left(\cdot, t_{0}\right)$ converges to -1 in the neighborhood of $r$. One can show (cf. Theorem 4) that this jump will contribute into the limiting energy $E$ an amount, greater than $C(\delta) \rho$, where $C$ is a constant depending 
only on $\delta$. But then, taking more points in $N_{t_{0}}^{\delta}$, passing to further subsequences in $\varepsilon$, and using the boundedness of the energy, we find that the set $N_{t_{0}}^{\delta} \cap[\rho, R]$ is at most finite.

By choosing any countable dense subset $G$ of $[0, \infty)$ and using the diagonal argument we can find a subsequence $\left\{\varepsilon_{j}\right\}_{j \in \mathbf{N}}$ such that the set $N_{t}^{\delta} \cap[\rho, R]$ is finite for every $t \in G$. Furthermore, for any sequence $\left\{r_{k}\right\}_{k \in \mathbf{N}}$ such that $\lim _{k \rightarrow \infty} r_{k}=0$ and any $l \in \mathbf{N}$, we can find a subsequence $\left\{\varepsilon_{j}\right\}_{j \in \mathbf{N}}$ such that the set $N_{t}^{\delta} \cap\left[r_{k}, R\right]$ is finite for every $t \in G$ and every $k=1, \ldots, l$. Then applying the diagonal argument again, we conclude that there exists a subsequence $\left\{\varepsilon_{j}\right\}_{j \in \mathbf{N}}$ for which the set $N_{t}^{\delta} \cap\left[r_{0}, R\right]$ is finite for every $t \in G$ and every $r_{0} \in(0, R)$. Clearly, the same statement holds for $P_{t}^{\delta}$.

We are now in a position to prove the following technical lemma describing the dynamics of $N_{t}^{\delta}$ and $P_{t}^{\delta}$. This lemma is somewhat similar to the clearing-out lemma (see [13], [14], [15], or [16]).

LEMmA 7. There exist a $\delta>0$ and a set $\Pi_{\delta} \subset[0, \infty)$ containing finitely many points characterized by the following.

Let $t_{0} \in[0, \infty) \backslash \Pi_{\delta}$ and $A \subset N_{t_{0}} \backslash N_{t_{0}}^{\delta}$ be an open set, where $N_{t_{0}}^{\delta}$ is as in Remark 13 . Then there exists an $\alpha\left(A, t_{0}\right)>0$ for which

$$
N_{t}^{\delta} \cap\left[r_{0}, R\right] \cap A=\varnothing
$$

for any $t \in\left[t_{0}, t_{0}+\alpha\left(A, t_{0}\right)\right)$ and any $r_{0} \in(0, R)$. The same result holds for $P_{t}^{\delta}$.

Proof. Let $T>0$ be given. Fix $\theta>0$. Since the limiting energy $E$ is a bounded, monotone decreasing function of $t$, there are at most finitely many points $t \in[0, T]$ such that $E\left(t^{-}\right)-E\left(t^{+}\right) \geq \theta / 2$, where $E\left(t_{i}^{ \pm}\right)=\lim _{t \rightarrow t^{ \pm}} E(t)$. Denote the set of such points by $\Pi_{\delta}$ (the meaning of the index $\delta$ will become evident later on). Then $[0, \infty) \backslash \Pi_{\delta}$ consists of finitely many intervals

$$
(0, \infty) \backslash \Pi_{\delta}=\bigcup_{i=1}^{n}\left(t_{i}, t_{i+1}\right) .
$$

Fix an arbitrary $i=1, \ldots, n$ and $\eta>0$ small. Then using the monotonicity and boundedness of $E$ we can subdivide an interval $\left[t_{i}+\eta, t_{i+1}-\eta\right]$ into finitely many intervals on which the variation of $E$ does not exceed $\theta / 2$ :

$$
\begin{gathered}
{\left[t_{i}+\eta, t_{i+1}-\eta\right]=\bigcup_{j=1}^{l}\left[\hat{t}_{j-1}, \hat{t}_{j}\right],} \\
E\left(\hat{t}_{j-1}^{+}\right)-E\left(\hat{t}_{j}^{-}\right)<\frac{\theta}{2} \quad \text { for } j=1, \ldots, l .
\end{gathered}
$$

Moreover, we have, for $\varepsilon$ sufficiently small, that the variation of $E^{\varepsilon}\left[u^{\varepsilon}\right]$ on each of these intervals does not exceed $\theta$. This follows from the pointwise convergence of $E^{\varepsilon}\left[u^{\varepsilon}\right]$ to $E$. Applying the procedure of Theorem 9, we find that

$$
\left|\varepsilon \lambda^{\varepsilon}(t)\right| \leq C(M) \theta^{1 / 2}+O(1)
$$

uniformly on $\bigcup_{j=1}^{l}\left[\hat{t}_{j-1}, \hat{t}_{j}\right]$, where $O(1) \rightarrow 0$ as $\varepsilon \rightarrow 0$. Fix a number

$$
t_{0} \in\left(t_{i}+\eta, t_{i+1}-\eta\right) \cap G \subset[0, \infty) \backslash \Pi_{\delta}
$$


and set

$$
\frac{\delta}{2}:=2 C(M) \theta^{1 / 2}
$$

where $\theta$ is chosen so that $2 \delta<1$. Then for $\varepsilon>0$ sufficiently small, $\left|\varepsilon \lambda^{\varepsilon}(t)\right| \leq \delta / 2$ uniformly on $\left[t_{i}+\eta, t_{i+1}-\eta\right]$.

Let $A$ be an open set such that $\bar{A} \subset N_{t_{0}} \backslash N_{t_{0}}^{\delta}$, and suppose without loss of generality that $A$ is an open interval that lies between two consecutive points in $N_{t_{0}}^{\delta}$ or between the point from $N_{t_{0}}^{\delta}$ and either $r_{0}$ or $R$ (this is possible since $t_{0} \in G$ ). Suppose that for every $t \in\left(t_{0}, t_{i+1}-\eta\right)$, there exists $\sigma \in\left(t_{0}, t\right]$ satisfying

$$
N_{\sigma}^{\delta} \cap A \neq \varnothing .
$$

In other words, there exists an $r_{\sigma} \in A$ and subsequences $\left\{\varepsilon_{j_{k}(\sigma)}\right\}_{k \in \mathbf{N}}$ and $\left\{p_{j_{k}(\sigma)}\right\}_{k \in \mathbf{N}}$ such that (omitting the index $j_{k}(\sigma)$ )

$$
\lim _{\substack{\varepsilon \rightarrow 0 \\ p \rightarrow r_{\sigma}}} u^{\varepsilon}(p, \sigma) \geq-\delta
$$

Fix $t \in\left(t_{0}, t_{i+1}-\eta\right)$. We can choose an open interval $B$ such that $\bar{B} \subset N_{t_{0}} \backslash N_{t_{0}}^{\delta}$ and $\bar{A} \subset B$. By our assumption and since $u^{*}\left(r, t_{0}\right)$ is upper semicontinuous, $u^{\varepsilon}\left(r, t_{0}\right) \leq$ $-\delta_{1}<-\delta$ uniformly on $\bar{B}$ for $\varepsilon>0$ small. Let $r_{A}^{ \pm}, r_{B}^{ \pm}$denote the endpoints of $A$ and $B$ correspondingly, that is, $A=\left(r_{A}^{-}, r_{A}^{+}\right)$while $B=\left(r_{B}^{-}, r_{B}^{+}\right)$. By (74) we can fix a small $\varepsilon>0$ such that there exists a $p \in A$ with $u^{\varepsilon}(p, \sigma)=-\delta_{2}$ for some $\delta \leq \delta_{2}<\delta_{1}$ such that $2 \delta_{2}<1$. We want to obtain a contradiction.

First, assume that one can find $\rho_{1} \in\left(r_{B}^{-}, p\right)$ and $\rho_{2} \in\left(p, r_{B}^{+}\right)$satisfying

$$
u^{\varepsilon}\left(\rho_{1}, s\right)<-\delta_{2} \quad \text { and } \quad u^{\varepsilon}\left(\rho_{2}, s\right)<-\delta_{2}
$$

for all $s \in\left[t_{0}, \sigma\right]$. Define $\tau \in\left[t_{0}, \sigma\right]$ by

$$
\tau=\min \left\{s \in\left[t_{0}, \sigma\right]: u^{\varepsilon}(r, s) \geq-\delta_{2} \text { for some } r \in\left(\rho_{1}, \rho_{2}\right)\right\} .
$$

From the continuity of $u^{\varepsilon}$, our initial assumption on $B$ and (75), it follows that $\tau \in\left(t_{0}, \sigma\right]$ and that $u^{\varepsilon}$ achieves its maximum on $\left(\rho_{1}, \rho_{2}\right) \times\left(t_{0}, \tau\right]$ at $\left(r_{\tau}, \tau\right)$ with $u^{\varepsilon}\left(r_{\tau}, \tau\right)=-\delta_{2}$. But at the point of the maximum we must have (following the usual proof of the maximum principle)

$$
0<-\frac{\delta}{2}+\delta_{2} \leq \varepsilon \lambda^{\varepsilon}(\tau)-u^{\varepsilon}\left(r_{\tau}, \tau\right) \leq 0,
$$

which is a contradiction. Therefore, at least one of the following conditions must be satisfied:

1. For every $r \in\left(r_{B}^{-}, p\right)$ there exists $s_{r} \in\left(t_{0}, \sigma\right]$ such that $u^{\varepsilon}\left(r, s_{r}\right)=-\delta_{2}$;

2. For every $r \in\left(p, r_{B}^{+}\right)$there exists $s_{r} \in\left(t_{0}, \sigma\right]$ such that $u^{\varepsilon}\left(r, s_{r}\right)=-\delta_{2}$. 
Assume, without loss of generality, that condition (77) holds. Then for every $r \in\left(p, r_{B}^{+}\right)$,

$$
\int_{t_{0}}^{t}\left|h\left(u^{\varepsilon}(r, s)\right)_{t}\right| d s \geq \int_{t_{0}}^{\sigma}\left|h\left(u^{\varepsilon}(r, s)\right)_{t}\right| d s \geq h\left(u^{\varepsilon}\left(r, t_{0}\right)\right)-h\left(-\delta_{2}\right)>0
$$

since $h$ is decreasing.

Following the proof of Theorem 3, we obtain the following estimate:

$$
\int_{\Omega} \int_{t_{1}}^{t_{2}}\left|h_{t}\left(u^{\varepsilon}\right)\right| d s d x \leq C(M)\left(t_{2}-t_{1}\right)^{1 / 2} \text { for any } t_{1}, t_{2} \in[0, \infty),
$$

where $M$ is given by (14.a). Then since $p \in A$,

$$
\begin{gathered}
\int_{r_{A}^{+}}^{r_{B}^{+}}\left(h\left(u^{\varepsilon}\left(r, t_{0}\right)\right)-h\left(-\delta_{2}\right)\right) r d r<\int_{p}^{r_{B}^{+}}\left(h\left(u^{\varepsilon}\left(r, t_{0}\right)\right)-h\left(-\delta_{2}\right)\right) r d r \\
\leq \int_{p}^{r_{B}^{+}} \int_{t_{0}}^{t}\left|h_{t}\left(u^{\varepsilon}\right)\right| r d s d r \leq C(M)\left(t-t_{0}\right)^{1 / 2} .
\end{gathered}
$$

Passing to a limit on a subsequence chosen in (74) and since $h(u)=\frac{(u)^{3}}{3}-u$ with $u^{\varepsilon} \rightarrow-1$ a.e. on $B \times\left\{t_{0}\right\}$,

$$
\left(\frac{2}{3}-\delta+\frac{\delta^{3}}{3}\right) \cdot\left(\left(r_{B}^{+}\right)^{2}-\left(r_{A}^{+}\right)^{2}\right) \leq 2 C(M)\left(t-t_{0}\right)^{1 / 2}
$$

The left-hand side of this inequality is strictly positive and independent of $t$, while the right-hand side can be made arbitrarily small by choosing $t$ sufficiently close to $t_{0}$. This is a contradiction and therefore, contrary to (73), there exists an $\alpha\left(A, t_{0}\right)>0$ such that $N_{t}^{\delta} \cap A=\varnothing$ for any $t \in\left[t_{0}, t_{0}+\alpha\left(A, t_{0}\right)\right)$. Since $\eta$ and $i$ in (70) are arbitrary and $G$ is dense in $[0, \infty)$, the conclusion of the lemma will hold for any $t \in[0, T] \backslash \Pi_{\delta}$. It also follows from the proof that $\alpha$ depends on a distance between $A$ and $N_{t_{0}}^{\delta}$. In fact, by (80),

$$
\alpha\left(A, t_{0}\right) \geq \min \left\{\inf _{t>t_{0}} \Pi_{\delta}-t_{0}, C\left(r_{0}\right) \operatorname{dist}^{2}\left(A, N_{t_{0}}^{\delta} \cup\left\{r_{0}, R\right\}\right)\right\}
$$

where $C\left(r_{0}\right)$ is a constant depending on $r_{0}$ and $\operatorname{dist}(A, B)$ is a distance between two sets $A, B \subset[0, R]$.

The generalization to $(69)$ is obvious.

From now on we will assume for $\Pi_{\delta}, N_{t}^{\delta}$, and $P_{t}^{\delta}$ that the number $\delta$ is as in the previous lemma.

Definition 2. Fix $t \geq 0$. Every point $r_{0} \in N_{t}^{\delta} \cup P_{t}^{\delta}$ will be called a "ghost" interface at the time $t$. We will denote the set $N_{t}^{\delta} \cup P_{t}^{\delta}$ of "ghost" interfaces by $\Gamma_{t}^{\prime}$.

From now on we will refer to the interfaces from $\Gamma_{t}$ (see Def. 1) as "regular" interfaces. Observe that $\Gamma_{t} \cap\left(N_{t} \cup P_{t}\right)=\varnothing$ and $\Gamma_{t} \cup\left(N_{t} \cup P_{t}\right)=(0, R)$, while $\Gamma_{t}^{\prime} \subset N_{t} \cup P_{t}$. In the remainder of this paper the type of the interface will not be specified for the results that hold for both "ghost" and "regular" interfaces. 

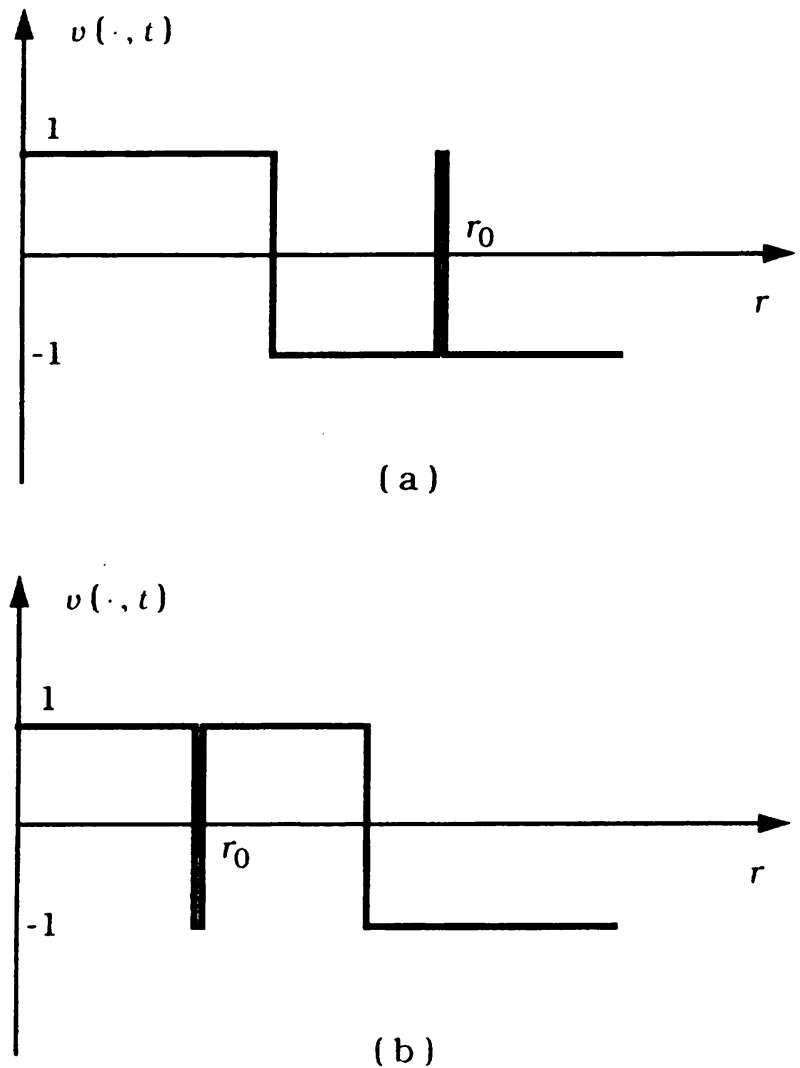

FIG. 1. The "ghost" interfaces: (a) $r_{0} \in N_{t}^{\delta}$; (b) $r_{0} \in P_{t}^{\delta}$

In addition, we would say that there is an interface at $r=0$ if

$$
u^{*}(0, t) \neq-u_{*}(0, t)
$$

and that there is an interface at $r=R$ if

$$
u^{*}(R, t) \neq-u_{*}(R, t) \text {. }
$$

Our definitions admit a simple geometric interpretation. Let $r \in(0, R)$ be such that in some neighborhood of $r$, the asymptotic limit $v$ of solutions of the nonlocal GinzburgLandau equations is equal to $u_{1}$ to the right of $r$ and to $u_{2}$ to the left of $r$, where $u_{1}$ and $u_{2}$ are constants. Then we will say that there is a "regular" interface at $r$ if $u_{1} \neq u_{2}$ and that there is a "ghost" interface at $r$ if $u_{1}=u_{2}=u$ but $v(r) \neq u$. In this formal setting one can think of a "ghost" interface as of a coupled pair of "regular" interfaces (see Fig. 1 ). We have that $u=-1$ for a "ghost" interface from $N_{t}^{\delta}$ and that $u=1$ for a "ghost" interface from $P_{t}^{\delta}$.

If $r \in[0, R]$ is an interface, by choosing a sufficiently small neighborhood $A$ of $r$, one can see that the variations of solutions $u^{\varepsilon}$ of the nonlocal Ginzburg-Landau equation over $A$ are bounded from below uniformly in $\varepsilon$ (cf. Theorem 4$)$. Therefore, for a small 
$\varepsilon>0$ a smooth function $u^{\varepsilon}$ might have a single or multiple "jumps" near $r$. Then it is customary to say that the multiplicity of the interface at $r$ is greater than one if $u^{\varepsilon}$ "jumps" at $r$ more than once. We will give the exact definition of the multiplicity later in this chapter.

Notice that the exact behavior of the solution near $r$ is not captured by our definitions of the interfaces, since we can only conclude that a "total variation of the limiting solution" near $r$ is greater than zero. It turns out, however, that for our method one does not need to know the multiplicity of a "regular" interface in order to determine its motion. Indeed, the viscosity method described in the previous chapter relies only on the fact that the sign of the limiting solution changes at $r$ and not on an inner structure of the interface. The notion of the multiplicity, nevertheless, will be taken into account when we describe the interactions of interfaces.

The next three corollaries are immediate consequences of Lemma 7.

COROLlary 1. The position functions of the "ghost" interfaces are continuous on their respective intervals of existence, except maybe the points from the set $\Pi_{\delta}$.

REMARK 14. By Theorem 3 the same result holds for "regular" interfaces for every $t>0$.

COROLlaRY 2. The "ghost" interfaces can nucleate only at exceptional times $t \in \Pi_{\delta}$.

Corollary 3. For each $t \in[0, T] \backslash \Pi_{\delta}$ and each closed set $A \subset N_{T} \backslash N_{t}^{\delta}$ there exists an $h>0$ such that, for an $\varepsilon>0$ small, $u^{\varepsilon}$ is uniformly negative on $A \times[t, t+h]$. The same result holds for $P_{t}^{\delta}$.

Then repeating the proof of Theorem 12 we obtain

TheOREm 17. Fix $r_{0} \in(0, R)$. Then $z^{\varepsilon}\left(\cdot, t_{0}\right) \rightarrow d\left(\cdot, t_{0}\right)$ uniformly on $\left[r_{0}, R\right]$ if $t_{0} \in$ $[0, T] \backslash \Pi_{\delta}$ is a continuity point of $E$. Here $d\left(\cdot, t_{0}\right)$ is a signed distance function to the set $\left(\Gamma_{t} \cup \Gamma_{t}^{\prime}\right) \cap\left[r_{0}, R\right]$.

REMARK 15 . Let $t_{0} \in[0, T] \backslash \Pi_{\delta}$ be a continuity point of the limiting energy $E$. Fix $\gamma>0$ and $r_{0} \in(0, R)$. Following the proof of Proposition 5 from [12], using (38) and the bound on $\left|\varepsilon \lambda^{\varepsilon}\right|$, we obtain that there exist an $\varepsilon_{0}>0$ and a $\tau>0$ such that

$$
\left(q^{\prime}\left(z^{\varepsilon} / \varepsilon\right)\right)^{2}\left(\left(z_{r}^{\varepsilon}\right)^{2}-1\right)<\gamma
$$

uniformly on $\left[r_{0}, R\right]$, where $\varepsilon<\varepsilon_{0}$ and $t \in\left[t_{0}-\tau, t_{0}+\tau\right] \subset[0, T] \backslash \Pi_{\delta}$.

REMARK 16. Suppose that $t_{0} \in[0, T] \backslash \Pi_{\delta}$ is a continuity point of the limiting energy $E$. Fix arbitrary $r_{0} \in(0, R)$. Let $\delta$ be as in Lemma 7 and choose $0<\gamma<\left(1-\delta^{2}\right)^{2}$. Then by Remark 15, we can find an $\varepsilon_{0}>0$ and a $\tau>0$ such that

$$
q^{\prime}\left(z^{\varepsilon}(r, t) / \varepsilon\right) \leq 1-\delta^{2},
$$

for every $r \in\left[r_{0}, R\right]$, satisfying the equation $z_{r}^{\varepsilon}(r, t)=0$, where $\varepsilon<\varepsilon_{0}$ and $t \in\left[t_{0}-\right.$ $\left.\tau, t_{0}+\tau\right]$. Now fix $\varepsilon<\varepsilon_{0}$. Let $r(t)$ be a continuous curve such that $(r(t), t)$ satisfies

$$
\begin{aligned}
z^{\varepsilon}(r, t) & \geq 0, \\
z_{r}^{\varepsilon}(r, t) & =0, \\
z_{r r}^{\varepsilon}(r, t) & \geq 0,
\end{aligned}
$$


for every $t \in\left[t_{1}, t_{2}\right] \subset\left(t_{0}-\tau, t_{0}+\tau\right)$. By (83), we have that

$$
u^{\varepsilon}(r(t), t)=q\left(z^{\varepsilon}(r(t), t) / \varepsilon\right)>\delta
$$

for every $t \in\left[t_{1}, t_{2}\right]$. Following the proof of Lemma 7 , we can choose $\varepsilon$ to be so small that $\left|\varepsilon \lambda^{\varepsilon}(t)\right| \leq \delta / 2$ uniformly on $\left[t_{1}, t_{2}\right]$. By Taylor's formula and (84) we obtain

$$
\begin{aligned}
z^{\varepsilon}(r(t & +\Delta t), t+\Delta t)-z^{\varepsilon}(r(t), t)=z_{r}^{\varepsilon}(r(t), t)(r(t+\Delta t)-r(t)) \\
& +z_{r r}^{\varepsilon}(\xi, t)(r(t+\Delta t)-r(t))^{2}+z_{t}^{\varepsilon}(r(t), t) \Delta t+o(\Delta t) \\
\geq & z_{t}^{\varepsilon}(r(t), t) \Delta t+o(\Delta t)
\end{aligned}
$$

where $\xi \in[r, r+\Delta t]$ and $\Delta t>0$ is small. At the same time, using the standard proof of the maximum principle, we conclude that

$$
z_{t}^{\varepsilon}(r(t), t) \geq \frac{\delta}{\varepsilon}
$$

for every $t \in\left[t_{1}, t_{2}\right]$. Thus, by (85),

$$
z^{\varepsilon}\left(r\left(t_{2}\right), t_{2}\right)-z^{\varepsilon}\left(r\left(t_{1}\right), t_{1}\right) \geq \frac{\delta}{\varepsilon}\left(t_{2}-t_{1}\right)
$$

and $t_{2}-t_{1}$ is of order $\varepsilon$ since $\left|z^{\varepsilon}\right|$ is uniformly bounded on $[0, \infty)$. Hence, by the maximum principle in the proof of Lemma 7 , there exists a $\tau>0$ such that there are no $(r, t) \in\left[r_{0}, R\right] \times\left(t_{0}-\tau, t_{0}+\tau\right]$ for which (84) holds. Then, since $r_{0}$ is arbitrary, there are no points in $(0, R]$ satisfying $(84)$ for $t=t_{0}$. A similar statement can be proven for the symmetric set of conditions

$$
\begin{aligned}
z^{\varepsilon}(r, t) & \leq 0, \\
z_{r}^{\varepsilon}(r, t) & =0 \\
z_{r r}^{\varepsilon}(r, t) & \leq 0 .
\end{aligned}
$$

Now the idea of multiplicity can be made more precise on the basis of Remarks 9 and 16. We remind the reader that, as a consequence of the uniform boundedness of $E^{\varepsilon}\left[u^{\varepsilon}\right]$, we have for any continuity point $t>0$ of the limiting energy $E$ that

$$
\left(q^{\prime}\left(z^{\varepsilon} / \varepsilon\right)\right)^{2}\left(\left(z_{r}^{\varepsilon}\right)^{2}-1\right) \rightarrow 0
$$

as $\varepsilon \rightarrow 0$ uniformly on $[0, R]$ (cf. Remark 15). Suppose now that $t_{0}$ is a continuity point of $E$ such that there exists a subsequence $\left\{\varepsilon_{n_{k}\left(t_{0}\right)}\right\}_{k=1}^{\infty}$ satisfying

$$
\lim _{k \rightarrow \infty}\left(\varepsilon_{n_{k}} \int_{0}^{R}\left(u_{t}^{\varepsilon_{n_{k}}}\right)^{2} r d r\right)<\infty
$$

The set of such points is dense in $[0, T]$ by Remark 6 . We would like to calculate the value of $E\left(t_{0}\right)$. Suppose, in addition, that there are finitely many interfaces at $t_{0}$ (this 
assumption will be justified later). Then Theorem 17 can be extended onto the interval $[0, R]$. Applying the technique of Sec. 6.2 , we find that

$$
\lim _{\varepsilon \rightarrow 0} E^{\varepsilon}\left[u^{\varepsilon}\right](t)=\lim _{\varepsilon \rightarrow 0} \int_{\Omega}\left|\nabla h\left(u^{\varepsilon}\right)\right| d x=\lim _{\varepsilon \rightarrow 0}\left\{\sum_{i=1}^{M\left(t_{0}\right)} \int_{r_{i}\left(t_{0}\right)-\delta}^{r_{i}\left(t_{0}\right)+\delta}\left|\nabla h\left(u^{\varepsilon}\right)\right| d x\right\},
$$

where $\delta>0$ is arbitrarily small, $r_{i}\left(t_{0}\right)$ is a position of the $i$ th interface ("regular" or "ghost") at the time $t_{0}$, and $M\left(t_{0}\right)$ is a total number of interfaces at $t_{0}$. In general, one would have to include into the right-hand side of (88) the possible contribution from the parts of the solution at $r=0$ and $r=R$. However, as we will see in the next section, there cannot exist an interface located exactly at the center of the domain $\Omega$, which makes a nonzero contribution to the energy. In fact, there is no interface at $r=0$ for $n=2$ and the multiplicity of the interface at $r=0$ has to be finite for $n=3$. The multiplicity of the interface at $r=R$ is always finite by the boundedness of the limiting energy. We will assume for notational simplicity that there is no interface at $r=R$ when $t=t_{0}$.

Fix now $\eta>0$ small. From (87) we have that there exists an $\varepsilon_{0}>0$ such that

$$
\left(q^{\prime}\left(z^{\varepsilon} / \varepsilon\right)\right)^{2}\left(\left(z_{r}^{\varepsilon}\right)^{2}-1\right)<\eta
$$

uniformly on $(0, R)$ if $\varepsilon<\varepsilon_{0}$. Therefore, $z^{\varepsilon}(\cdot, t)$ is strictly monotone on the intervals where $\left(q^{\prime}\left(z^{\varepsilon} / \varepsilon\right)\right)^{2}<\eta$, that is, where

$$
\left(u^{\varepsilon}\right)^{2}=\left(q\left(z^{\varepsilon} / \varepsilon\right)\right)^{2}<1-\eta^{1 / 2} .
$$

Since $u^{\varepsilon}$ is continuous, one can deduce that the set $\left\{\left(u^{\varepsilon}\right)^{2}<1-\eta^{1 / 2}\right\}$ consists of the open intervals $I_{\alpha}^{\varepsilon}$ such that for every $\alpha$ the function $u^{\varepsilon}$ is monotone on $I_{\alpha}^{\varepsilon}$ (cf. [12]). Each $I_{\alpha}^{\varepsilon}$ contains exactly one zero of $u^{\varepsilon}$ and the variation of $u^{\varepsilon}$ on $I_{\alpha}^{\varepsilon}$ is $2\left(1-\eta^{1 / 2}\right)^{1 / 2}=2-O(\eta)$. Then the variation of $h\left(u^{\varepsilon}\right)$ on any given $I_{\alpha}^{\varepsilon}$ is $\frac{4}{3}-O(\eta)$ and therefore, by (88), the number of the intervals $I_{\alpha}^{\varepsilon}$ should be uniformly bounded in $\varepsilon$. Using Remark 16, we can also conclude for a small $\varepsilon>0$ that there is exactly one zero of $z_{r}^{\varepsilon}$ between any two consecutive intervals $I_{\alpha}^{\varepsilon}$. Hence the variation of $h\left(u^{\varepsilon}\right)$ on the set $[0, R] \backslash \bigcup_{\alpha} I_{\alpha}^{\varepsilon}$ is of the order $\eta$ for every $\varepsilon<\varepsilon_{0}$. It should be clear from (88) that for every $\delta>0$ we can choose an $\varepsilon_{1}<\varepsilon_{0}$ such that the set $\bigcup_{\alpha} I_{\alpha}^{\varepsilon}$ is contained in the $\bigcup_{i=1}^{M\left(t_{0}\right)}\left(r_{i}\left(t_{0}\right)-\delta, r_{i}\left(t_{0}\right)+\delta\right)$ if $\varepsilon<\varepsilon_{1}$. Then passing, if necessary, to a subsequence, we conclude for any given $i=1, \ldots, M\left(t_{0}\right)$ that the number $m_{i}\left(t_{0}\right)$ of the intervals $I_{\alpha}^{\varepsilon}$ inside $\left(r_{i}\left(t_{0}\right)-\delta, r_{i}\left(t_{0}\right)+\delta\right)$ is constant in $\varepsilon$, if $\varepsilon>0$ is small. Since $\delta$ is arbitrary, the jump set of the limiting solution is located at $r_{i}\left(t_{0}\right)$ where $i=1, \ldots, M\left(t_{0}\right)$. Then we will say that multiplicity of the interface at $r_{i}\left(t_{0}\right)$ is $m_{i}\left(t_{0}\right)$. By letting $\varepsilon \rightarrow 0$ in (88) we obtain that

$$
E\left(t_{0}\right)=\frac{8}{3} \pi \sum_{i=1}^{M\left(t_{0}\right)} m_{i}\left(t_{0}\right) r_{i}\left(t_{0}\right)
$$

The preceding result shows for a small $\varepsilon>0$ and $\rho>0$, that there are exactly as many zeroes of $z_{r}^{\varepsilon}$ on $[\rho, R] \times\left\{t_{0}\right\}$ as one needs to approximate a function $v$ with $\sum_{i=1}^{M\left(t_{0}\right)} m_{i}\left(t_{0}\right)$ 
jumps inside $[\rho, R]$. Moreover, for $n=2,3$, the number of zeroes of $z_{r}^{\varepsilon}$ will be finite for every $t>t_{0}$, since it is finite at $t_{0}$ (cf. Theorem 8). Hence, the number $\sum_{i=1}^{M\left(t_{0}\right)} m_{i}(t)$ of the interfaces (counted with their multiplicities) will remain finite for every $t>t_{0}$, when $\Omega \subset \mathbf{R}^{2}$ or $\mathbf{R}^{3}$. In the next section we will generalize this result using the methods of measure theory.

Thus, we can formally say that the limiting solution $v$ has finitely many "jumps" between +1 and -1 clustered at interfaces. On a scale $\varepsilon$ these jumps are located infinitely far away from each other, from the boundary, and from the center of $\Omega$. This is a consequence of the relation (87). The number of jumps of $v$ at an interface constitutes its multiplicity. A given interface $r$ is a "regular" interface if the "total variation" of $v$ across $r$ is an odd integer and it is a "ghost" interface if the "total variation" of $v$ across $r$ is an even integer. Later, we will see that the multiplicity of any interface cannot increase for all but finitely many $t>0$ unless it collides with another interface.

Before attempting to describe the dynamics of moving interfaces, we would like to determine whether new interfaces can nucleate in the interior of the set $\Omega \backslash\left(\Gamma_{t} \cup \Gamma_{t}^{\prime}\right)$ or at the center of the domain $\Omega$. So far, we only know from Lemma 7 , that nucleation cannot occur for any $(r, t) \in\left[r_{0}, R\right] \times[0, T] \backslash \Pi_{\delta}$. The interfaces, however, may appear from the origin $r=0$ if $t \geq 0$, or at any $r \in[0, R]$ if $t \in \Pi_{\delta}$. We will rule out both of these possibilities in the next section.

7.2. The absence of nucleation. In Lemma 7 we determined that new interfaces cannot appear in the interior of the set $\left[r_{0}, R\right] \backslash\left(\Gamma_{t} \cup \Gamma_{t}^{\prime}\right)$ for any $r_{0}>0$ and any time $t \notin \Pi_{\delta}$. We want to show that, in fact, the same conclusion is true for every $t>0$ with $r_{0}=0$. Some of the results below depend on the dimension of the space containing the domain $\Omega$; hence we will assume that $\Omega \subset \mathbf{R}^{n}$. We begin by proving the following estimate.

Lemma 8. There exist an $\varepsilon_{0}>0$ and an $\alpha>0$ such that for any $0<\varepsilon<\varepsilon_{0}$ and any $t>0$ one can find an $r(\varepsilon, t) \in[0, R]$ for which

$$
\frac{W(u(r(\varepsilon, t), t))}{\varepsilon} \leq \alpha^{2}
$$

and

$$
\left|\int_{B_{r(\varepsilon, t)}} \nabla h\left(u^{\varepsilon}\right) \bullet \mathbf{x} d x\right| \geq \frac{n}{4}\left(h(-1)\left\|B_{R}\right\|-H\right)>0
$$

hold, where $H=\left|\int_{\Omega} h\left(u^{\varepsilon}\right) d x\right|$ and $B_{r}$ is the ball of radius $r$ and volume $\left\|B_{r}\right\|$ centered at the origin.

Proof. Fix $t>0$. Due to the uniform boundedness of the energy we have that the Lebesgue measure

$$
\mathcal{L}\left\{x \in \Omega \mid \frac{W\left(u^{\varepsilon}(x, t)\right)}{\varepsilon}>\frac{\alpha^{2}}{2}\right\}<\frac{2 M}{\alpha^{2}}
$$

for every $\alpha>0$ and $\varepsilon>0$. Here $M$ is as in (14.a). Choose $\rho>0$ such that

$$
h(-1)\left(\left\|B_{R}\right\|-\left\|B_{\rho}\right\|\right) \leq \frac{1}{4}\left(h(-1)\left\|B_{R}\right\|-H\right) .
$$

The right-hand side of this inequality is strictly positive by our assumption on initial data. 
Let $\alpha$ satisfy

$$
\frac{2 M}{\alpha^{2}}<\frac{1}{2}\left(\left\|B_{R}\right\|-\left\|B_{\rho}\right\|\right)
$$

Then, by $(92)$, there exists $r(\varepsilon, t) \in(\rho, R)$ such that (90) holds. Moreover, by the definition of $W$,

$$
\left(u^{\varepsilon}(r(\varepsilon, t), t)\right)^{2}>1-\alpha \sqrt{\varepsilon} .
$$

Denote $p_{\varepsilon}:=u^{\varepsilon}(r(\varepsilon, t), t)$ and $w_{\varepsilon}=\sqrt{1-\alpha \sqrt{\varepsilon}}$. Then, integrating by parts, using (93) and the monotonicity of $h$, we obtain for a small $\varepsilon>0$ that

$$
\begin{aligned}
& \left|\int_{B_{r(\varepsilon, t)}} \nabla h\left(u^{\varepsilon}\right) \bullet \mathbf{x} d x\right| \geq n\left|\left\|B_{r(\varepsilon, t)}\right\| h\left(p_{\varepsilon}\right)-\int_{B_{r(\varepsilon, t)}} h\left(u^{\varepsilon}\right) d x\right| \\
& \quad \geq n\left\{\left\|B_{r(\varepsilon, t)}\right\| h\left(-w_{\varepsilon}\right)-\left(H+h(-1)\left\|B_{R}\right\|-h(-1)\left\|B_{r(\varepsilon, t)}\right\|\right)\right\} \\
& \quad \geq n\left\{\left(h\left(-w_{\varepsilon}\right)-h(-1)\right)\left\|B_{r(\varepsilon, t)}\right\|+\left(2 h(-1)\left\|B_{\rho}\right\|-H-h(-1)\left\|B_{R}\right\|\right)\right\} \\
& \quad \geq n\left\{\left(h\left(-w_{\varepsilon}\right)-h(-1)\right)\left\|B_{r(\varepsilon, t)}\right\|-\frac{1}{2}\left(h(-1)\left\|B_{R}\right\|-H\right)\right\} .
\end{aligned}
$$

Then, since $h$ is continuous and $\lim _{\varepsilon \rightarrow 0} w_{\varepsilon}=1$, we can find $\varepsilon_{0}$ such that

$$
\left(h(-1)-h\left(-w_{\varepsilon}\right)\right)\left\|B_{R}\right\| \leq-\frac{1}{4}\left(h(-1)\left\|B_{R}\right\|-H\right),
$$

for every $\varepsilon<\varepsilon_{0}$ and hence (91) follows.

This technical result enables us to obtain an $L^{2}$-bound on $\lambda^{\varepsilon}$.

TheOREM 18. We have

$$
\varlimsup_{\varepsilon \rightarrow 0} \int_{t_{0}}^{t_{1}}\left(\lambda^{\varepsilon}\right)^{2} d s<C\left[\left(E\left(t_{0}\right)-E\left(t_{1}\right)\right)+\left(t_{1}-t_{0}\right)\right]
$$

for any $0 \leq t_{0}<t_{1}<\infty$. Then for every $T>0$ the set $\left\{\lambda^{\varepsilon}\right\}_{\varepsilon<\varepsilon_{0}}$ is uniformly bounded in $L^{2}([0, T])$.

Proof. Fix $0 \leq t_{0}<t_{1}<\infty$. Multiply Eq. (12) by $\nabla u^{\varepsilon} \bullet \mathbf{x}$ and integrate by parts twice over $B_{r(\varepsilon, t)}$ to obtain

$$
\begin{aligned}
\int_{B_{r(\varepsilon, t)}} \varepsilon u_{t}^{\varepsilon}\left(\nabla u^{\varepsilon} \bullet \mathbf{x}\right) d x-\int_{B_{r(\varepsilon, t)}} & {\left[(n-2) \frac{\varepsilon\left|\nabla u^{\varepsilon}\right|^{2}}{2}+n \frac{W\left(u^{\varepsilon}\right)}{\varepsilon}\right] d x } \\
& +\lambda^{\varepsilon} \int_{B_{r(\varepsilon, t)}}\left(\nabla h\left(u^{\varepsilon}\right) \bullet \mathbf{x}\right) d x+r(\varepsilon, t) \int_{\partial B_{r(\varepsilon, t)}}\left[\frac{W\left(u^{\varepsilon}\right)}{\varepsilon}-\frac{\varepsilon\left|\nabla u^{\varepsilon}\right|^{2}}{2}\right] d \sigma=0 .
\end{aligned}
$$

Solve this equation for $\lambda^{\varepsilon}$, take the square and integrate in $t$ over $\left[t_{1}, t_{2}\right]$. Then, since for $\varepsilon>0$ the coefficient in front of $\lambda^{\varepsilon}$ is uniformly bounded from below in $\varepsilon$ and $t$, we obtain by $(22)$

$$
\begin{aligned}
& \int_{t_{1}}^{t_{2}}\left(\int_{B_{r(\varepsilon, t)}} \varepsilon u_{t}^{\varepsilon}\left(\nabla u^{\varepsilon} \bullet \mathbf{x}\right) d x\right)^{2} d t \leq \int_{t_{1}}^{t_{2}}\left(\int_{\Omega} \varepsilon u_{t}^{\varepsilon}\left(\nabla u^{\varepsilon} \bullet \mathbf{x}\right) d x\right)^{2} d t \\
& \quad \leq R^{2} \int_{t_{1}}^{t_{2}}\left(\int_{\Omega} \frac{\varepsilon\left|\nabla u^{\varepsilon}\right|^{2}}{2} d x\right)\left(\int_{\Omega} \varepsilon\left(u_{1}^{\varepsilon}\right)^{2} d x\right) d t \leq M R^{2} \int_{t_{1}}^{t_{2}} \int_{\Omega} \varepsilon\left(u_{t}^{\varepsilon}\right)^{2} d x d t \\
& \quad \leq M R^{2}\left(E^{\varepsilon}\left[u^{\varepsilon}\left(\cdot, t_{1}\right)\right]-E^{\varepsilon}\left[u^{\varepsilon}\left(\cdot, t_{2}\right)\right]\right) .
\end{aligned}
$$


The remaining terms in (95), except the one with $\lambda^{\varepsilon}$, are uniformly bounded on $[0, \infty)$. Then (94) follows by the previous lemma. Hence, due to the uniform boundedness of the limiting energy, the set $\left\{\lambda^{\varepsilon}\right\}_{\varepsilon<\varepsilon_{0}}$ is uniformly bounded in $L^{2}([0, T])$ for every $T>0$.

As a next step, we define for every $t>0$ a measure

$$
\mu^{\varepsilon}(A ; t)=\int_{\Omega}\left(\frac{\varepsilon\left|\nabla u^{\varepsilon}\right|^{2}}{2}+\frac{W\left(u^{\varepsilon}\right)}{\varepsilon}\right) d x
$$

on Borel subsets of $\Omega$. Choose a smooth nonnegative function $\psi$ with a compact support in $\Omega$. Then, after integrating by parts and completing the square, we obtain

$$
\begin{aligned}
\frac{d}{d t} \int_{\Omega} \psi \mu^{\varepsilon}(d x ; t) & =-\varepsilon \int_{\Omega}\left(\nabla \psi \bullet \nabla u^{\varepsilon}\right) u_{t}^{\varepsilon} d x+\varepsilon \int_{\Omega} \psi u_{t}^{\varepsilon}\left[-\Delta u^{\varepsilon}+\frac{1}{\varepsilon^{2}} W^{\prime}\left(u^{\varepsilon}\right)\right] d x \\
& =-\varepsilon \int_{\Omega}\left(\nabla \psi \bullet \nabla u^{\varepsilon}\right) u_{t}^{\varepsilon} d x+\varepsilon \int_{\Omega} \psi u_{t}^{\varepsilon}\left[\frac{1}{\varepsilon} \lambda^{\varepsilon} h^{\prime}\left(u^{\varepsilon}\right)-u_{t}^{\varepsilon}\right] d x \\
& \leq \varepsilon \int_{\Omega} \frac{\left(\nabla \psi \bullet \nabla u^{\varepsilon}\right)^{2}}{2 \psi} d x+4\left(\lambda^{\varepsilon}\right)^{2} \int_{\Omega} \frac{W\left(u^{\varepsilon}\right)}{\varepsilon} \psi d x .
\end{aligned}
$$

Then

$$
\frac{d}{d t} \int_{\Omega} \psi \mu^{\varepsilon}(d x ; t) \leq C(\psi) \mu^{\varepsilon}(\{\psi>0\} ; t)+4\left(\lambda^{\varepsilon}\right)^{2} \int_{\Omega} \psi \mu^{\varepsilon}(d x ; t),
$$

which implies by Gronwall's inequality that

$$
\int_{\Omega} \psi \mu^{\varepsilon}(d x ; t) \leq C_{1} t+C_{2} \int_{\Omega} \psi \mu^{\varepsilon}(d x ; 0)
$$

where $C_{1}$ depends on $\psi$, the uniform bound on the energy, and the $L^{2}$-bound on $\lambda^{\varepsilon}$, while $C_{2}$ depends on the $L^{2}$-bound on $\lambda^{\varepsilon}$ only. Hence, using the weak-* compactness of Radon measures and (98), we can apply the diagonal argument to construct a subsequence $\left\{\varepsilon_{n}\right\}_{n \in \mathbf{N}}$ and a Radon measure $\mu$ such that (omitting the index $n$ )

$$
\lim _{\varepsilon \rightarrow 0} \int_{\Omega} \psi \mu^{\varepsilon}(d x ; t)=\int_{\Omega} \psi \mu(d x ; t)
$$

This procedure originates in Brakke [16] and has been used by several authors ([13], [15]). We refer the reader to [15] for the detailed treatment.

REMARK 17 . One can easily check that

$$
\left(\Gamma_{t} \cup \Gamma_{t}^{\prime}\right) \times[0,2 \pi) \subset \text { support of } \mu
$$

for every $t>0$. Moreover, using Theorem 17 , we find that at continuity points of the limiting energy

$$
\left(\Gamma_{t} \cup \Gamma_{t}^{\prime}\right) \times[0,2 \pi)=\text { support of } \mu .
$$

We are now ready to show that new interfaces cannot nucleate at the center of $\Omega$. 
ThEOREM 19. Let a continuity point $t_{0}$ of the limiting energy $E$ be such that there are finitely many interfaces when $t=t_{0}$. Then no new interfaces will appear from the center of the domain $\Omega$ for any $t \in\left(t_{0}, t_{1}\right)$, where $t_{1}=\inf _{t>t_{0}}\left(\Pi_{\delta} \cup \Xi_{t_{0}}\right)$ and $t \in \Xi_{t_{0}} \subset\left(t_{0}, \infty\right)$ if and only if there exists an interface that shrinks to the center of $\Omega$ at time $t$.

Proof. Let $t_{0} \in[0, T] \backslash \Pi_{\delta}$ be such that there are finitely many interfaces at $t=t_{0}$. Denote $r_{0}:=r_{1}\left(t_{0}\right)$ to be a position of the innermost interface at time $t_{0}$. Choose a positive, smooth, monotone decreasing function $\Psi$ with a compact support in $\Omega$ such that

$$
\Psi(r, \theta)= \begin{cases}1, & r \in\left[0, r_{0} / 3\right] \\ 0, & r>2 r_{0} / 3\end{cases}
$$

Then by (97)

$$
\begin{aligned}
\frac{d}{d t} \int_{\Omega} \Psi \mu^{\varepsilon}(d x ; t) & \leq 4\left(\lambda^{\varepsilon}\right)^{2} \int_{\Omega} \Psi \mu^{\varepsilon}(d x ; t)+\varepsilon \int_{\Omega} \frac{\left|\nabla u^{\varepsilon}\right|^{2}}{2} \Phi d x \\
& \leq 4\left(\lambda^{\varepsilon}\right)^{2} \int_{\Omega} \Psi \mu^{\varepsilon}(d x ; t)+\int_{\Omega} \Phi \mu^{\varepsilon}(d x ; t),
\end{aligned}
$$

where $\Phi=\frac{|\nabla \Psi|^{2}}{\Psi}$. It is easily checked that $\Phi$ is a continuous nonnegative function with a compact support in $\left[r_{0} / 3,2 r_{0} / 3\right] \times[0,2 \pi)$. Using Gronwall's inequality, we obtain from (101) that

$$
\begin{aligned}
\int_{\Omega} \Psi \mu^{\varepsilon}(d x ; t) \leq & \left(\int_{\Omega} \Psi \mu^{\varepsilon}\left(d x ; t_{0}\right)\right) \exp \left(4 \int_{t_{0}}^{t}\left(\lambda^{\varepsilon}(s)\right)^{2} d s\right) \\
& \quad+\int_{t_{0}}^{t} \int_{\Omega} \Phi \exp \left(4 \int_{s}^{t}\left(\lambda^{\varepsilon}(\sigma)\right)^{2} d \sigma\right) \mu^{\varepsilon}(d x ; s) d s \\
\leq C & {\left[\int_{\Omega} \Psi \mu^{\varepsilon}\left(d x ; t_{0}\right)+\int_{t_{0}}^{t} \int_{\Omega} \Phi \mu^{\varepsilon}(d x ; s) d s\right], }
\end{aligned}
$$

where $C$ depends only on the uniform $L^{2}$-bound on $\lambda^{\varepsilon}$. As $\varepsilon \rightarrow 0$, we have that

$$
\int_{\Omega} \Psi \mu^{\varepsilon}\left(d x ; t_{0}\right) \rightarrow \int_{\Omega} \Psi \mu\left(d x ; t_{0}\right)=0
$$

by our assumption on $t_{0}$. At the same time, due to the uniform boundedness of the energy,

$$
\int_{t_{0}}^{t} \int_{\Omega} \Phi \mu^{\varepsilon}(d x ; s) d s \rightarrow \int_{t_{0}}^{t} \int_{\Omega} \Phi \mu(d x ; s) d s .
$$

By Lemma 7 , one can find $\alpha\left(t_{0}, r_{0}\right)$ such that there are no interfaces on $\left[r_{0} / 6,5\left(r_{0} / 6\right)\right]$ when $t \in\left[t_{0}, t_{0}+\alpha\left(t_{0}, r_{0}\right)\right)$. Recall that

$$
\alpha\left(t_{0}, r_{0}\right)=\min \left\{\inf _{t>t_{0}} \Pi_{\delta}-t_{0}, C\left(r_{0}\right) \operatorname{dist}^{2}\left(A, N_{t_{0}}^{\delta} \cup\{0\}\right)\right\},
$$

by (81), where $A=\left[r_{0} / 6,5\left(r_{0} / 6\right)\right]$. Since the support of $\Phi$ is contained inside $A$, Theorem 17 implies that

$$
\int_{\Omega} \Phi \mu(d x ; s)=0
$$


whenever $s \in\left[t_{0}, t_{0}+\alpha\left(t_{0}, r_{0}\right)\right)$ is a continuity point of $E$. Therefore,

$$
\int_{t_{0}}^{t} \int_{\Omega} \Phi \mu^{\varepsilon}(d x ; s) d s \rightarrow 0
$$

as $\varepsilon \rightarrow 0$ when $t \in\left[t_{0}, t_{0}+\alpha\left(t_{0}, r_{0}\right)\right)$, and

$$
\int_{\Omega} \Psi \mu(d x ; t)=0
$$

for every $t \in\left[t_{0}, t_{0}+\alpha\left(t_{0}, r_{0}\right)\right)$. Thus we conclude, by the definition of $\Psi$, that there are no interfaces on $\left[0, r_{0} / 3\right]$ when $t \in\left[t_{0}, t_{0}+\alpha\left(t_{0}, r_{0}\right)\right)$. Then the statement of the theorem follows by (102).

Next we would like to extend this result to all $t>t_{0}$. We will need the following theorem.

THEOREM 20. Let $\tilde{t}>0$ be such that the positions of interfaces are uniformly bounded away from zero on an interval $[\tilde{t}-\sigma, \tilde{t})$ for some $\sigma>0$. Then no new interfaces can nucleate at the center of $\Omega$ when $t=\tilde{t}$.

Proof. Let $s$ and $\tau$ be such that $\tilde{t}-\sigma<\tau<\tilde{t}<s$ and both $\tilde{t}-\tau$ and $s-\tilde{t}$ are small. By our assumptions, the number of interfaces at $t=\tau$ is finite. Suppose that $\tau$ is a continuity point of the limiting energy $E$. Let $r_{0}(t)$ be a position of the innermost interface at time $t$, where $t \in[\tau, \tilde{t})$. Denote $\tilde{r}=\frac{1}{2} \liminf _{t \rightarrow \tilde{t}} r_{0}(t)$. Then, by our assumptions, $\tilde{r}>0$. Let

$$
\psi(x(r), t, s)= \begin{cases}1, & r<\tilde{r}, t<s \\ 1-t h\left(\frac{(r-\tilde{r})^{2}}{8(s-t)}\right), & r>\tilde{r}, t<s .\end{cases}
$$

Then

$$
\begin{aligned}
& \frac{d}{d t} \int_{\Omega} \psi \mu^{\varepsilon}(d x ; t)=\int_{\Omega} \psi_{t} \mu^{\varepsilon}(d x ; t)+\varepsilon \int_{\Omega} \frac{\left(\nabla \psi \bullet \nabla u^{\varepsilon}\right)^{2}}{2 \psi} d x+4\left(\lambda^{\varepsilon}\right)^{2} \int_{\Omega} \frac{W\left(u^{\varepsilon}\right)}{\varepsilon} \psi d x \\
& \quad \leq \varepsilon \int_{\Omega} \frac{\left|\nabla u^{\varepsilon}\right|^{2}}{2}\left(\psi_{t}+\frac{|\nabla \psi|^{2}}{\psi}\right) d x+\int_{\Omega} \frac{W\left(u^{\varepsilon}\right)}{\varepsilon}\left(4\left(\lambda^{\varepsilon}\right)^{2} \psi+\psi_{t}\right) d x \\
& \quad \leq 4\left(\lambda^{\varepsilon}\right)^{2} \int_{\Omega} \psi \mu^{\varepsilon}(d x ; t)
\end{aligned}
$$

for any $t<s$. This inequality follows from (97) since

$$
\psi_{t}+\frac{|\nabla \psi|^{2}}{\psi} \leq 0
$$

for every $t<s$. Therefore,

$$
\int_{\Omega} \psi \mu^{\varepsilon}(d x ; t) \leq\left[\int_{\Omega} \psi \mu^{\varepsilon}(d x ; \tau)\right] \exp \left(4 \int_{\tau}^{t}\left(\lambda^{\varepsilon}(s)\right)^{2} d s\right) \leq C \int_{\Omega} \psi \mu^{\varepsilon}(d x ; \tau),
$$

where $C$ depends on an $L^{2}$-bound on $\lambda^{\varepsilon}$. Passing to the limit as $\varepsilon \rightarrow 0$, we obtain for every $t \in[\tau, s)$ that

$$
\int_{\Omega} \psi(x, t, s) \mu(d x ; t) \leq C \int_{\Omega} \psi(x, \tau, s) \mu(d x ; \tau) .
$$


By the definition of $r_{0}$ we can choose $\tau$ to be such that $2 \tilde{r}-r_{0}(t)<\tilde{r} / 2$. Then, using the definition of $\psi$, we obtain

$$
\int_{\Omega} \psi(x, \tau, s) \mu(d x ; \tau)=O\left(1-t h\left(\frac{(\tilde{r})^{2}}{32(s-\tau)}\right)\right)=O\left(\exp \left(-\frac{(\tilde{r})^{2}}{16(s-\tau)}\right)\right)
$$

by evaluating the integral $\int_{\Omega} \psi \mu^{\varepsilon}(d x ; \tau)$ directly and passing to the limit in $\varepsilon$. The righthand side of (105) can be made arbitrarily small by taking $s$ and $\tau$ sufficiently close to each other. Therefore, we can find $\tau<\tilde{t}<s$ such that

$$
\int_{\Omega} \psi(x, t, s) \mu(d x ; t)<\frac{4 \pi \tilde{r}}{18}
$$

when $t \in[\tau, s)$. This implies that there are no interfaces on the interval $\left(\frac{\tilde{r}}{6}, \tilde{r}\right)$ for every continuity point $t$ of the limiting energy $E$ such that $t \in[\tau, s)$. But then, one can use the technique of the previous theorem to conclude that no interfaces can appear from the center of $\Omega$ for any $t \in[\tau, s)$.

In order to combine the conclusions of Theorems 19 and 20, we have to study the dependence of the multiplicity of interfaces on time. In addition, this will enable us to describe possible singularities of the nonlocal flow and determine the points in $\Omega$ where such singularities might occur.

For any $a, b, \gamma, s>0$ and $t<s$ let

$$
\psi_{a, b}(x(r), t, s)= \begin{cases}1-t h\left(\frac{(r-a)^{2}}{8(s-t)}\right), & 0<r<a, \\ 1, & a \leq r \leq b \\ 1-t h\left(\frac{(r-b)^{2}}{8(s-t)}\right), & b<r \leq R\end{cases}
$$

and

$$
\psi_{\gamma}(x(r), t, s)= \begin{cases}1-t h\left(\frac{(2(R-r)-\gamma)^{2}}{32(s-t)}\right), & 0 \leq r<R-\frac{\gamma}{2}, \\ 1, & R-\frac{\gamma}{2} \leq r \leq R .\end{cases}
$$

We start by making the following remark.

REMARK 18. By choosing the appropriate $a$ and $b$ in (106), one can apply the proof of Theorem 20 to $\psi_{a, b}$ to conclude that new interfaces cannot nucleate in the interior of the set $\left[r_{0}, R\right] \backslash\left(\Gamma_{t} \cup \Gamma_{t}^{\prime}\right)$ for any $t \in \Pi_{\delta}$. Therefore, Lemma 7 can be extended to all $t>0$. Now let $t_{0}$ be a continuity point of the limiting energy $E$ such that there are no interfaces on $[R-\gamma, R]$ for $t=t_{0}$ and some $\gamma>0$. Then, once again following the proof of Theorem 20, we have for $\psi_{\gamma}$, given by (107),

$$
\varlimsup_{\varepsilon \rightarrow 0} \int_{\Omega} \psi_{\gamma}(x, t, s) \mu^{\varepsilon}(d x ; t)=O\left(\exp \left(-\frac{1}{16 \gamma}\right)\right)
$$

whenever $t \in\left[t_{0}, t_{0}+\gamma\right]$. By evaluating the same integral explicitly we determine that there are no interfaces on $[R-\gamma, R]$ for $t \in\left[t_{0}, t_{0}+\gamma\right]$ and, therefore, for all $t>t_{0}$. Thus we will assume for simplicity that there are no interfaces on $[R-\gamma, R]$ for all $t>t_{0}$. 
Theorem 21. Suppose that $t_{0} \in[0, \infty) \backslash \Pi_{\delta}$ is a continuity point of the limiting energy $E$ such that there are finitely many interfaces at the time $t=t_{0}$. Then there exists $s>t_{0}$ such that the "total multiplicity"

$$
L(t)=\sum_{k=1}^{M(t)} m_{k}(t)
$$

of interfaces in $\Omega$ is nonincreasing on continuity points of $E$ in $\left[t_{0}, s\right)$. Here $M(t)$ is the number of interfaces at the time $t$.

Proof. Let $s>t_{0} \geq 0$ be given. By (103) and the definition (106) of $\psi_{a, b}$ we obtain that

$$
\int_{\Omega} \psi_{a, b}(x, t, s) \mu(d x ; t) \leq\left[\int_{\Omega} \psi_{a, b}\left(x, t_{0}, s\right) \mu(d x ; \tau)\right] \exp \left(4 \limsup _{\varepsilon \rightarrow \infty} \int_{t_{0}}^{t}\left(\lambda^{\varepsilon}(s)\right)^{2} d s\right)
$$

as $\varepsilon \rightarrow \infty$ for every $t_{0} \leq t<s$. Suppose that $\beta>1$ is given. Let

$$
\Delta_{\beta}:=\{t \in[0, \infty) \mid \exp \{C[(E(t)-E(s))+(s-t)]\} \geq \beta, \forall s>t\}
$$

where $C$ is as defined in (94). Then the monotonicity of the limiting energy implies that the set $\Delta_{\beta}$ is finite on $[0, \infty)$. Therefore, by Theorem 18 , for every $t_{0} \in[0, \infty) \backslash \Delta_{\beta}$ we can choose a number $s>t_{0}$ such that

$$
\int_{\Omega} \psi_{a, b}(x, t, s) \mu(d x ; t) \leq \beta \int_{\Omega} \psi_{a, b}\left(x, t_{0}, s\right) \mu\left(d x ; t_{0}\right),
$$

for all $t_{0} \leq t<s$. We will suppose for simplicity that for any number $\beta$ below, the set $\Delta_{\beta} \subset \Pi_{\delta}$.

Now fix $t_{0} \in[0, \infty) \backslash \Pi_{\delta}$, a continuity point of the limiting energy $E$ such that there are finitely many interfaces at the time $t=t_{0}$. Then, by Theorem 19 , the number of interfaces remains finite until the time $t_{1}$, where

$$
t_{1}=\inf _{t>t_{0}}\left(\Pi_{\delta} \cup \Xi_{t_{0}}\right)
$$

is the least time greater than $t_{0}$ such that either some interfaces shrink to the center of $\Omega$ at $t=t_{1}$ or $t_{1} \in \Pi_{\delta}$. Since the number $M\left(t_{0}\right)$ of interfaces at the time $t_{0}$ is finite, the interfaces are bounded away from zero and each has a finite multiplicity. For every continuity point $t \geq 0$ of the limiting energy $E$ denote

$$
L(t)=\sum_{k=1}^{M(t)} m_{k}(t)
$$

to be the "total multiplicity" of interfaces at the time $t$. Then the number $L\left(t_{0}\right)$ is finite. Let $\beta=\frac{2 L\left(t_{0}\right)+1}{2 L\left(t_{0}\right)}$. Suppose that $\rho>0$ is such that the intervals $\left(r_{k}\left(t_{0}\right)-\rho, r_{k}\left(t_{0}\right)+\rho\right)$, where $k=1, \ldots, M\left(t_{0}\right)$, are mutually disjoint. Using the continuity of interfaces, we can find a number $s>t_{0}$ such that interfaces will remain inside the set $\bigcup_{k=1}^{M\left(t_{0}\right)}\left(r_{k}\left(t_{0}\right)-\right.$ 
$\rho, r_{k}\left(t_{0}\right)+\rho$ ) for every $t_{0} \leq t \leq s$. Let $a=r_{k}\left(t_{0}\right)-\rho$ and $b=r_{k}\left(t_{0}\right)+\rho$. Fix $i \in\left\{1, \ldots, M\left(t_{0}\right)\right\}$ and consider the interface $r_{i}\left(t_{0}\right)$. Assume that $s>t_{0}$ is such that (109) is satisfied for every $t_{0} \leq t<s$ and that $\rho>0$ is such that $\frac{L\left(t_{0}\right) \rho}{r_{i}\left(t_{0}\right)}<\frac{1}{4}$. If $t_{0} \leq t<s$ is a continuity point of the limiting energy $E$ then, using the definition of $\psi_{a, b}$, we obtain by directly evaluating the integrals in (109)

$$
\sum m_{i_{k}}(t) r_{i_{k}}(t) \leq \frac{2 L\left(t_{0}\right)+1}{2 L\left(t_{0}\right)} m_{i}\left(t_{0}\right) r_{i}\left(t_{0}\right)+o\left(s-t_{0}\right)
$$

for every $t_{0} \leq t<s$. Here the sum in $i_{k}$ on the right-hand side of the inequality is taken over the interfaces that might exist at the time $t$ inside the interval $\left(r_{i}(t)-\rho, r_{i}(t)+\rho\right)$. Each of these interfaces must originate at $\left(r_{i}\left(t_{0}\right), t_{0}\right)$, since the positions of interfaces are continuous on $[0, \infty) \backslash \Pi_{\delta}$. Then, by choosing $s$ to be sufficiently close to $t_{0}$ we obtain

$$
\sum m_{i_{k}}(t) \leq \frac{2 L\left(t_{0}\right)+1}{2 L\left(t_{0}\right)} m_{i}\left(t_{0}\right)+\frac{L\left(t_{0}\right) \kappa}{r_{i}\left(t_{0}\right)}+o\left(s-t_{0}\right)<\frac{2 L\left(t_{0}\right)+1}{2 L\left(t_{0}\right)} m_{i}\left(t_{0}\right)+\frac{1}{2} .
$$

Suppose that the sum on the right-hand side of (111) is greater than $m_{i}\left(t_{0}\right)$. Then

$$
m_{i}\left(t_{0}\right)+1<\frac{2 L\left(t_{0}\right)+1}{2 L\left(t_{0}\right)} m_{i}\left(t_{0}\right)+\frac{1}{2}
$$

and therefore,

$$
L\left(t_{0}\right)<m_{i}\left(t_{0}\right)
$$

which contradicts the definition of $L\left(t_{0}\right)$. Hence $\sum m_{i_{k}}(t) \leq m_{i}\left(t_{0}\right)$ for every continuity point $t$ of the limiting energy $E$ such that $t_{0} \leq t<s$. We conclude that the sum of the multiplicities of interfaces with an initial point at $\left(r_{i}\left(t_{0}\right), t_{0}\right)$ is less than or equal to the multiplicity of the interface $r_{i}$ at the time $t_{0}$. Moreover, since the number of interfaces is finite at $t=t_{0}$, there exists a number $s>t_{0}$ such that the "total multiplicity" $L$ of interfaces, defined by (108), is nonincreasing on continuity points of the limiting energy $E$ in $\left[t_{0}, s\right)$.

REMARK 19. Let $s$ be the same as in the previous theorem and choose $\tau<s<s_{1}$, such that $\tau$ is a continuity point of $E$, while both $s_{1}-s$ and $s-\tau$ are small. Then, using the same analysis as in the proof of the previous theorem, we conclude that $L$ is nonincreasing on continuity points of the limiting energy $E$ in some $\left[\tau, s_{1}\right)$ where $s \in\left[\tau, s_{1}\right)$. Furthermore, we obtain for any given $i=1, \ldots, M(s)$ that the sum of the multiplicities of interfaces meeting at $r_{i}(s)$ is always greater than or equal to the sum of the multiplicities of interfaces with the initial point at $r_{i}(s)$.

This procedure can be continued for as long as no new interfaces can appear at the center of the domain $\Omega$. Therefore, $L$ is nonincreasing on continuity points of the limiting energy $E$ in $\left[t_{0}, t_{1}\right)$, where $t_{1}$ is given by (110).

Since the multiplicity of every interface is a positive integer, the function $L(t)$, defined on a set $D \subset\left[t_{0}, t_{1}\right)$ of continuity points of the limiting energy $E$, is positive and integervalued. Since $L$ is nonincreasing on $D$ and $D$ is dense in $\left[t_{0}, t_{1}\right]$, we can extend $L$ to the interval $\left[t_{0}, t_{1}\right)$ by assuming for every $t \in\left[t_{0}, t_{1}\right)$ that

$$
L(t)=\lim _{s_{j} \rightarrow t^{+}} L\left(s_{j}\right),
$$

where $\left\{s_{j}\right\}_{j \in \mathbf{N}} \subset D$. 
Now suppose that $t_{1} \notin \Pi_{\delta}$, that is, $t_{1} \in \Xi_{t_{0}}$ and one of the interfaces shrinks to the center of $\Omega$ at the time $t_{1}$. Here $\Xi_{t_{0}}$ is defined in Theorem 19. Then, by the continuity of interfaces, there are finitely many interfaces at the time $t=t_{1}$ since $L$ is nonincreasing on $\left[t_{0}, t_{1}\right)$. Therefore, we can continue applying the procedure of Remark 19 until we reach the first time $t_{2}$ such that $t_{2} \in \Pi_{\delta}$. Moreover, using the same remark, we conclude that $L$ remains nonincreasing on continuity points of the limiting energy $E$ in $\left[t_{0}, t_{2}\right)$. By (112), we can extend $L$ to a bounded, positive, nonincreasing, and integer-valued function on $\left[t_{0}, t_{2}\right)$. It follows then that $L$ can be discontinuous at most at finitely many points in $\left[t_{0}, t_{2}\right)$.

Due to the continuity of interfaces, Remark 18, and the boundedness of $L$, the number of interfaces in $\Omega$ is uniformly bounded on the interval $\left[t_{2}-\sigma, t_{2}\right]$ for any small $\sigma>0$. Let $r_{0}(t)$ be a position of the innermost interface at the time $t$. Then $r_{0}$ is continuous on $\left[t_{2}-\sigma, t_{2}\right) \subset[0, T] \backslash \Pi_{\delta}$, except maybe for finitely many points. Indeed, the positions of interfaces are continuous on their respective intervals of existence inside $[0, T] \backslash \Pi_{\delta}$. Therefore, if $r_{0}$ is discontinuous at some $t \in\left[t_{2}-\sigma, t_{2}\right)$, the interface closest to the origin disappears at $t$. Then, by Theorem 20 , the function $L$ is discontinuous at $t$, but there are only finitely many such points in $t \in\left[t_{2}-\sigma, t_{2}\right)$. Hence we can assume that $\tau$ is such that $r_{0}$ is continuous on $\left[t_{2}-\sigma, t_{2}\right)$. Suppose that $\tilde{r}=\frac{1}{2} \lim _{t \rightarrow \tilde{t}} r_{0}(t)>0$. Then we can apply Theorem 20 to conclude that no new interfaces can appear from the center of $\Omega$ at $t_{2}$, and use a slightly modified version of Theorem 21 to conclude that the "total multiplicity" $L$ might increase at $t_{2}$, but it will remain bounded.

If, at the time $t_{2}$, some interface shrinks to the center of the domain $\Omega$ then $\tilde{r}=0$. However, using the boundedness of $L$, the proof of Theorem 20 can be easily extended to such $t_{2}$ by a small modification in the definition of $\tilde{r}$.

Continuing in a similar manner, while taking into account the finiteness of the set $\Pi_{\delta}$, the continuity of interfaces, and (112), we prove the following theorem.

Theorem 22. Suppose that there are finitely many interfaces at some time $t_{0}>0$, where $t_{0}$ is a continuity point of the limiting energy $E$. Then

1) The interfaces cannot nucleate at the center of the domain $\Omega$ for any $t>t_{0}$.

2 ) The "total multiplicity" $L$ of interfaces, given by (108) and (112), is nonincreasing on $\left[t_{0}, \infty\right) \backslash \Pi_{\delta}$ and bounded on $\left[t_{0}, \infty\right)$. The number $M(t)$ of interfaces at a time $t$ is uniformly bounded on $\left[t_{0}, \infty\right)$.

3) For any $i=1, \ldots, M(t)$ and $t \in\left[t_{0}, \infty\right] \backslash \Pi_{\delta}$, the sum of multiplicities of the interfaces meeting at $r_{i}(t)$ is always greater than or equal to the sum of multiplicities of the interfaces that originate at $r_{i}(t)$.

Here the uniform boundedness of $M(t)$ follows from the boundedness of $L$ since the multiplicities of interfaces are nonnegative and integer-valued.

Theorem 22 implies that new interfaces in $\Omega$ can only appear for $t>t_{0}$ following the splitting of an interface with multiplicity greater than one. Consider, for example, a "ghost" interface of multiplicity two that can be formally modeled by a coupled pair of "regular" interfaces with the jumps of the same size and the opposite sign. Then, depending on the relative speed of the interfaces in the pair, the "ghost" interface might either disappear or split up into two distinct "regular" interfaces. 
The preceding discussion enables us to say that the positions of interfaces are backward continuous on $\left[t_{0}, \infty\right)$. That is, if $a \in(0, R)$ is a position of some interface at $t_{1}>t_{0}$, then there exists a continuous curve $p$ such that $p\left(t_{1}\right)=a$ and $p(t)$ is an interface for every $t \in\left[t_{0}, t_{1}\right]$.

We conclude this section by proving the following lemma.

Lemma 9. Let $x \in \mathbf{R}^{n}$ where $n=2,3$. Then there exists a dense subset $A$ of $(0, \infty)$ such that for every $t \in A$ one can find a $t$-dependent subsequence $\left\{\varepsilon_{j}\right\}_{j \in \mathbf{N}}$ such that the asymptotic limit of $u^{\varepsilon_{j}}$ as $j \rightarrow \infty$ has finitely many interfaces at the time $t$.

Proof. Let $A$ be as defined in Remark 6 and fix $t \in A$. We have to show that at the time $t$ interfaces cannot concentrate at $r=0$. Without loss of generality, we can prove this lemma for $n=2$. In the estimate (51) set $y=0$ and choose $y_{1}>0$ such that for $t \in A$

$$
\frac{W\left(u^{\varepsilon}\left(y_{1}, t\right)\right)}{\varepsilon} \rightarrow 0 \quad \text { as } \varepsilon \rightarrow 0 .
$$

Then using the boundary conditions on $u^{\varepsilon},(51)$ can be rewritten as

$$
\frac{W\left(u^{\varepsilon}(0, t)\right)}{\varepsilon} \leq \varepsilon \int_{0}^{y_{1}} u_{t}^{\varepsilon} u_{r}^{\varepsilon} d r-\varepsilon \int_{0}^{y_{1}}\left(u_{r}^{\varepsilon}\right)^{2} \frac{d r}{r}+C\left(M, a_{t}\right)
$$

for a small $\varepsilon>0$. Here $C\left(M, a_{t}\right)$ is a constant, $M$ is the uniform bound on $E^{\varepsilon}\left[u^{\varepsilon}\right]$, and $a_{t}$ is defined in Remark 6 . Consequently, by Hölder's inequality and for a small $\varepsilon>0$

$$
\begin{aligned}
0 & <\frac{W\left(u^{\varepsilon}(0, t)\right)}{\varepsilon} \\
& \leq\left(\varepsilon \int_{0}^{y_{1}}\left(u_{r}^{\varepsilon}\right)^{2} \frac{d r}{r}\right)^{1 / 2}\left(\varepsilon \int_{0}^{y_{1}}\left(u_{t}^{\varepsilon}\right)^{2} r d r\right)^{1 / 2}-\varepsilon \int_{0}^{y_{1}}\left(u_{r}^{\varepsilon}\right)^{2} \frac{d r}{r}+2 C\left(M, a_{t}\right) \\
& \leq a_{t}^{1 / 2}\left(\varepsilon \int_{0}^{y_{1}}\left(u_{r}^{\varepsilon}\right)^{2} \frac{d r}{r}\right)^{1 / 2}-\varepsilon \int_{0}^{y_{1}}\left(u_{r}^{\varepsilon}\right)^{2} \frac{d r}{r}+2 C\left(M, a_{t}\right),
\end{aligned}
$$

and therefore

$$
\varepsilon \int_{0}^{y_{1}}\left(u_{r}^{\varepsilon}\right)^{2} \frac{d r}{r} \leq C\left(M, a_{t}\right)
$$

Now we can use (51) to show that

$$
\int_{0}^{\delta} \int_{y}^{\delta} \frac{W\left(u^{\varepsilon}\right)}{\varepsilon} d r d y
$$

is of order $\varepsilon$. One can prove using this estimate, that Lemma 4 holds for every $t \in A$. It follows then from the bound (113), that no infinite sequence of the "regular" interfaces with radii convergent to zero can exist at the time $t \in A$. This is a consequence of the equipartition of the energy (52) and (99). Indeed, suppose that "regular" interfaces concentrate at the center of $\Omega$ at the time $t$. Then applying the technique that was used to prove (89), we conclude that the right-hand side of (113) can be made arbitrarily large for a small $\varepsilon>0$. This, however, contradicts (113). Therefore, on a dense subset $A$ of $[0, T]$ there are at most finitely many "regular" interfaces. Since the positions of 
"ghost" interfaces at the time $t$ may depend on a subsequence in $\varepsilon$, the same result can be extended to "ghost" interfaces by passing to a further $t$-dependent subsequence.

REMARK 20. It immediately follows from (113) that for $n=2$, the interface cannot be located at the center of the domain $\Omega$ if $t \in A$. For $n=3$ the analog of (113) implies that the multiplicity of the interface at $r=0$ is finite. Finally, for $n>3$, we can use the method of the previous lemma to prove that on a dense subset $A$ of $[0, T]$ an interface of arbitrary multiplicity cannot be located exactly at the center of $\Omega$ and have a nonzero contribution to the energy at the same time. It is easy to observe that, otherwise, both

$$
\alpha(t)<\varepsilon \int_{0}^{y}\left(u_{r}^{\varepsilon}\right)^{2} r^{n-1} d r \leq \beta(t)
$$

and

$$
\varepsilon \int_{0}^{y}\left(u_{r}^{\varepsilon}\right)^{2} r^{n-3} d r \leq \beta(t)
$$

are satisfied uniformly in $\varepsilon$, where $0<\alpha(t), \beta(t)<\infty$ for every $t \in A$ and $y>0$. Then, applying the technique that was used to prove formula (89), we find that $\varepsilon \int_{0}^{y}\left(u_{r}^{\varepsilon}\right)^{2} r^{n-3} d r$ must be arbitrarily large for $\varepsilon>0$ small, if there is an interface at $r=0$ such that (114) holds. This, however, contradicts (115). The same results can be extended to all continuity points of the limiting energy $E$ by using the continuity of interfaces and the monotonicity of $E$.

Lemma 9, along with the previous theorems, implies that for $\Omega$ in $\mathbf{R}^{2}$ or $\mathbf{R}^{3}$ and arbitrarily small $t>0$, we can find a subsequence $\left\{\varepsilon_{j}\right\}_{j \in \mathbf{N}}$ such that there are finitely many interfaces at the time $t$. Then the number of interfaces is uniformly bounded on $[t, \infty)$. Similar results should be valid for the spaces of higher dimensions. However, we are unable to prove this.

Since our analysis in the next chapter will rely on the finiteness of the number of interfaces for every $t>0$, we will assume for $\Omega \subset \mathbf{R}^{n}, n>3$, that, at least on a subsequence, the initial data has finitely many interfaces.

7.3. The dynamics of interfaces. We have already determined in (89) that on a dense subset $A$ of $[0, T]$ the limiting energy $E$ can be computed by the formula

$$
E(t)=\frac{8}{3} \pi \sum_{i=1}^{M} m_{i}(t) r_{i}(t)
$$

Fix any $\sigma>0$. By our assumptions on the initial data, we obtain for an appropriate subsequence, that the asymptotic limit $v$ of solutions $u^{\varepsilon}$ of (12) can be characterized by moving interfaces, the number of which is uniformly bounded on $[\sigma, \infty)$. Here $\sigma$ can be set equal to zero if the number of interfaces is finite at $t=0$. Hence we will assume without loss of generality that $\sigma=0$.

Let $L(t)$ be the "total multiplicity" of interfaces at the time $t$, as defined in (108) and (112). By (108)

$$
L(t)=\sum_{k=1}^{M(t)} m_{k}(t)
$$


for every continuity point $t$ of the limiting energy $E$. By Theorem 22 and the discussion before it, the function $L$ is bounded, positive, integer-valued on $[0, \infty)$ and nonincreasing on $[0, \infty) \backslash \Pi_{\delta}$. Thus, $L$ can be discontinuous at no more than finitely many points in $[0, \infty)$. Denote the set of these points as $\Pi$.

Suppose that $t \in[0, \infty)$ is such that $L$ is continuous at $t$. Then, by the continuity of interfaces, the monotonicity of the limiting energy $E$, Theorem 22 , and (116), we have that $E$ is continuous at $t$ and therefore can be determined by (116). Hence, $E$ is continuous on $[0, \infty) \backslash \Pi$.

Observe that $\Pi_{\delta} \subset \Pi$. We would like to describe the singular behavior of interfaces for $t \in \Pi$. Fix any $t \in \Pi$. Then $L$ is discontinuous at $t$. By Theorem 22 and the monotonicity of the limiting energy, either of the following happens at $t$ :

(a) For some $i \in\{1, \ldots, M(t)\}$, the sum of the multiplicities of the interfaces that meet at $\left(r_{i}(t), t\right)$ is strictly greater than the sum of the multiplicities of interfaces that originate at $\left(r_{i}(t), t\right)$. Then the limiting energy $E$ is discontinuous at $t$.

(b) For some $i \in\{1, \ldots, M(t)\}$, the interface $r_{i}$ shrinks to the center of $\Omega$ at the time $t$. Then the limiting energy $E$ remains continuous at $t$.

(c) Both (a) and (b) occur at the same time.

In particular, (a) occurs when two interfaces collide at $\left(r_{i}(t), t\right)$ and disappear.

Clearly, the set $\Pi$ does not contain all times when geometric singularities of the nonlocal flow might occur. Indeed, suppose for some $t_{0} \in[0, T] \backslash \Pi$ and $i=1, \ldots, M\left(t_{0}\right)$ that the interface $r_{i}\left(t_{0}\right)$ has the multiplicity $m_{i}\left(t_{0}\right)>1$. Then the sum of multiplicities of the interfaces that may originate at $r_{i}\left(t_{0}\right)$ is equal to $m_{i}\left(t_{0}\right)$. In other words, for $\varepsilon \ll 1$, the solution of the nonlocal Ginzburg-Landau equation has $m_{i}\left(t_{0}\right)$ "jumps" near $r_{i}\left(t_{0}\right)$. Then formally, by taking different combinations of these "jumps", we can interpret the interface $r_{i}\left(t_{0}\right)$ as being composed from several interfaces of multiplicity lower than $m_{i}\left(t_{0}\right)$. It is possible that for $t>t_{0}$, the interfaces in this composition will drift apart, causing the original interface to split up. Then, there exists an interval $\left[t_{0}, t_{0}+\sigma\right] \subset[0, T] \backslash \Pi$ for some $\sigma>0$ such that the sum of the multiplicities of the new interfaces with the initial point at $r_{i}\left(t_{0}\right)$ is equal to $m_{i}\left(t_{0}\right)$. Observe that the limiting energy $E$ is continuous at $t_{0}$. In fact, by repeating the arguments of Theorem 15 , we can prove that $E$ is locally Lipschitz continuous on $[0, T] \backslash \Pi$.

Later, the same interfaces might recombine, forming the interface of the multiplicity $m_{i}\left(t_{0}\right)$, then split again, etc. Therefore, even on the intervals where the limiting energy is continuous, the behavior of the interfaces might be extremely complicated, with numerous geometric singularities of the nonlocal flow. Using the continuity of interfaces we can show, however, that the set of times in $[0, T] \backslash \Pi$ corresponding to such singularities is nowhere dense in $[0, T] \backslash \Pi$. Indeed, by the definition of $\Pi$, the sum $L(t)=\sum_{i=1}^{M I(t)} m_{i}(t)$ is independent of time on the interval $\left[t_{1}, t_{2}\right]$ if $\left[t_{1}, t_{2}\right] \subset \Pi$. Suppose for simplicity that $L(t)=3$. Then there are three "jumps" $A, B$, and $C$ from which the interfaces on $\left[t_{1}, t_{2}\right]$ are composed. These "jumps" can be arranged into the following combinations:

1) $A, B$, and $C$-three "regular" interfaces;

2) $A B$ and $C$-one "ghost" and one "regular" interface;

3) $A$ and $B C$-one "regular" and one "ghost" interface;

4) $A B C$-one "regular" interface of multiplicity three. 
Let $\Delta_{k}$ be the set of times in $\left[t_{1}, t_{2}\right]$ at which the combination $k$ exists. Then $\left[t_{1}, t_{2}\right]=$ $\bigcup_{k=1}^{4} \Delta_{k}$. Suppose that the set $\Delta_{1}^{c} \cap \Delta_{2}^{c}$ has a nonempty interior. Choose $\Delta \subset \Delta_{1}^{c} \cap \Delta_{2}^{c}$ open. Then, since the interfaces are continuous on $\left[t_{1}, t_{2}\right]$, only the combination (2) of $A B$ and $C$ can exist on $\Delta$ and $\Delta \cap \Delta_{1}=\varnothing$. This is a contradiction. Hence, $\Delta_{1}^{c} \cap \Delta_{2}^{c}$ has an empty interior. By repeating the same argument, we find that the set $\bigcup_{k, l=1}^{4} \Delta_{k}^{c} \cap \Delta_{l}^{c}$ is nowhere dense in $\left[t_{1}, t_{2}\right]$.

Therefore, in any neighborhood of every $t \in[0, T] \backslash \Pi$ there exists a $t_{0} \in[0, T] \backslash \Pi$ and $\alpha\left(t_{0}\right)>0$ such that there are no geometric singularities of the nonlocal flow on the interval $\left(t_{0}, t_{0}+\alpha\left(t_{0}\right)\right)$. In Sec. 7.4 we will show that, in fact, "regular" interfaces cannot split up for any $t \in[0, T] \backslash \Pi$ and that the "regular" interfaces appearing after the splitting of a "ghost" interface at some $t=t_{0}$ cannot recombine for any $t>t_{0}$. Therefore, the number of times when "ghost" interfaces may split up into "regular" interfaces is finite, because the number of interfaces is uniformly bounded on $[0, \infty)$. By including these times into the set $\Pi$, we will obtain that $\alpha\left(t_{0}\right)=\min _{t \in \Pi}\left(t-t_{0}\right)$.

Suppose again for simplicity that $x \in \mathbf{R}^{2}$. We know that at an arbitrary time $t_{0} \in$ $[0, T] \backslash \Pi$ there are finitely many interfaces of either kind- "regular" or "ghost". We would like to show that the evolution of every "regular" interface depends in a nonlocal fashion on its mean curvatures. Let $t_{0}$ be such that there exists a number $\alpha\left(t_{0}\right)$ such that $\left[t_{0}, t_{0}+\alpha\left(t_{0}\right)\right] \subset[0, T] \backslash \Pi$ and the positions of all interfaces are represented by continuous and mutually disjoint curves $r_{i}$ on $\left[t_{0}, t_{0}+\alpha\left(t_{0}\right)\right]$ for $i=1, \ldots, N+K$. Here $N$ is a number of "regular" interfaces and $K$ is a number of "ghost" interfaces on the interval $\left[t_{0}, t_{0}+\alpha\left(t_{0}\right)\right]$. Then, for every $i=1, \ldots, N+K$, the functions $r_{i}(\cdot)$ are uniformly bounded from below on $\left[t_{0}, t_{0}+\alpha\left(t_{0}\right)\right]$ by some $\rho>0$, and no "ghost" or "regular" interface can disappear or change its multiplicity on that interval. Since there are finitely many interfaces for every $t \in\left[t_{0}, t_{0}+\alpha\left(t_{0}\right)\right]$, we can set $r_{0}=0$ in Theorem 17. Then, with a few changes, the results of Sec. 6.2 still hold on $\left[t_{0}, t_{0}+\alpha\left(t_{0}\right)\right)$.

Recall that for any $h>0$

$$
d_{h}=\frac{1}{h} \int_{t}^{t+h} d(r, s) d s
$$

Then, since $d$ is known explicitly, we have for $h$ small enough, that near the "regular" interfaces either

$$
d_{h}(r, t)=r-\frac{1}{h} \int_{t}^{t+h} r_{i}(s) d s, \text { if } d_{r}\left(r_{i}(t), t\right)>0 \text { for } t \in\left(t_{0}, t_{0}+\alpha\left(t_{0}\right)-\gamma\right)
$$

or

$$
d_{h}(r, t)=\frac{1}{h} \int_{t}^{t+h} r_{i}(s) d s-r, \text { if } d_{r}\left(r_{i}(t), t\right)<0 \text { for } t \in\left(t_{0}, t_{0}+\alpha\left(t_{0}\right)-\gamma\right),
$$

for some arbitrarily small $\gamma \in\left(0, \alpha\left(t_{0}\right)\right)$ such that $h<\gamma$ and $i=1, \ldots, N$. The time interval is restricted to $\left(t_{0}, t_{0}+\alpha\left(t_{0}\right)-\gamma\right)$ so that (117) and (118) make sense. For every "regular" interface set

$$
r_{i h}(t):=\frac{1}{h} \int_{t}^{t+h} r_{i}(s) d s
$$

where $i=1, \ldots, N$. Following the proof of Theorem 16 we obtain 
Theorem 23. Choose $\left\{h_{n}\right\}_{n \in \mathbf{N}}$ for which the conclusion of Remark 10 holds. For $\gamma \in(0, \alpha(t))$ small, denote $\Omega_{\alpha}=[0, R] \times\left(t_{0}, t_{0}+\alpha\left(t_{0}\right)-\gamma\right)$. Assume that $\phi \in C^{\infty}\left(\Omega_{\alpha}\right)$ is such that $d_{h_{n}}-\phi$ has a maximum at $\left(r_{0}, t_{0}\right)$ and $d_{h_{n}}\left(r_{0}, t_{0}\right)<0$. Then

$$
\phi_{t}-\Delta \phi+\lambda_{h_{n}} \leq 0 \text { at }\left(r_{0}, t_{0}\right),
$$

where $\lambda_{h_{n}}$ is as in Remark 10 .

Finally, by repeating the arguments following Theorem 17, we conclude that for every $h$ small (omitting the index $n$ in $h_{n}$ )

(a) if $d_{r}\left(r_{i}(t), t\right)>0$ for $t \in\left(t_{0}, t_{0}+\alpha\left(t_{0}\right)-\gamma\right)$, then $r_{i h}$ is a viscosity solution of

$$
\dot{r}_{i h}+\frac{1}{r_{i h}}-\lambda_{h}=0 \quad \text { on }\left(t_{0}, t_{0}+\alpha\left(t_{0}\right)-\gamma\right) ;
$$

(b) if $d_{r}\left(r_{i}(t), t\right)<0$ for $t \in\left(t_{0}, t_{0}+\alpha\left(t_{0}\right)-\gamma\right)$, then $r_{i h}$ is a viscosity solution of

$$
\dot{r}_{i h}+\frac{1}{r_{i h}}+\lambda_{h}=0 \quad \text { on }\left(t_{0}, t_{0}+\alpha\left(t_{0}\right)-\gamma\right),
$$

where $\lambda_{n} \in C\left(\left[t_{0}, t_{0}+\alpha\left(t_{0}\right)\right]\right)$ and $r_{i}$ are the positions of "regular" interfaces for $i=$ $1 \ldots, N$. Recall that, by Remark 10 , the set $\left\{\lambda_{h}\right\}_{n \in \mathbf{N}}$ is bounded in $C\left(\left[t_{0}, t_{0}+\alpha\left(t_{0}\right)-\gamma\right]\right)$ for any $\gamma \in\left(0, \alpha\left(t_{0}\right)\right)$.

Since the positions of all interfaces are known at the time $t=t_{0}$, then so are the initial conditions on $r_{i h}$ for every $i=1, \ldots, N$. Thus, given $\lambda_{h}$, Eqs. (119) and (120) have the unique classical and, therefore, viscosity solutions on $\left[t_{0}, t_{0}+\alpha\left(t_{0}\right)-\gamma\right)$.

Now we can prove the following theorem describing the motion of "regular" interfaces.

THEOREM 24. Let $r_{i}$ denote the positions of "regular" interfaces for $i=1, \ldots, N$. Fix any $\gamma \in\left(0, \alpha\left(t_{0}\right)\right)$. There exists $\lambda \in L^{\infty}\left(\left[t_{0}, t_{0}+\alpha\left(t_{0}\right)-\gamma\right]\right)$ such that on $\left[t_{0}, t_{0}+\alpha\left(t_{0}\right)-\gamma\right]$ the function $r_{i}$ is a weak (in a sense of distributions) solution of

$$
\dot{r}_{i}+\frac{1}{r_{i}}-\lambda=0 \quad \text { if } d_{r}\left(r_{i}(t), t\right)>0,
$$

and

$$
\dot{r}_{i}+\frac{1}{r_{i}}+\lambda=0 \quad \text { if } d_{r}\left(r_{i}(t), t\right)<0, \text { where } i=1, \ldots, N .
$$

Moreover, $\lambda$ satisfies

$$
\int_{t_{1}}^{t_{2}}\left(\sum_{i=1}^{N} r_{i}(s)\right) \lambda(s) d s=a_{N}\left(t_{2}-t_{1}\right), \quad \text { for any } t_{1}, t_{2} \in\left[t_{0}, t_{0}+\alpha\left(t_{0}\right)-\gamma\right]
$$

where

$$
a_{N}(t)=\frac{1}{2}\left[\operatorname{ap} \lim _{r \rightarrow R} v(r, t)-\operatorname{ap} \lim _{r \rightarrow 0} v(r, t)\right] .
$$

Here ap $\lim _{r \rightarrow 0} v(r, t)$ is the approximate limit of $v(\cdot, t)$ as $r \rightarrow a$ (for the definition of the approximate limit see, for example, [27]). 
Notice that (123) depends neither on the multiplicity of "regular" interfaces nor on the positions of "ghost" interfaces. Indeed, as we will see, (123) is a consequence of the volume-preserving character of the nonlocal flow and therefore is independent of the behavior of the limiting solution on a set $\Gamma_{t} \cup \Gamma_{t^{\prime}}$ of measure zero.

Proof. Let $A:=\left[t_{0}, t_{0}+\alpha\left(t_{0}\right)-\gamma\right]$. Since $\left\|\lambda_{h_{n}}\right\|_{L^{\infty}(A)} \leq C$ for every $n \in \mathbf{N}$, then on a subsequence $h \rightarrow 0$ (omitting the index $n$ in $h_{n}$ )

$$
\lambda_{h} \stackrel{*}{\rightarrow} \lambda \text { weakly- } * \text { in } L^{\infty}(A) \text { for some } \lambda \in L^{\infty}(A)
$$

We also know that when $h \rightarrow 0$,

$$
r_{i_{h}} \rightarrow r_{i} \text { uniformly on } A \text {, }
$$

by definition of $r_{i h}$ for $i=1, \ldots, N$. Then, since $r_{i h}$ are uniformly bounded away from zero on $A$ for every $i=1, \ldots, N$,

$$
\frac{1}{r_{i h}} \rightarrow \frac{1}{r_{i}} \text { uniformly on } A \text { for } i=1, \ldots, N
$$

Hence, by (121) and (122)

$$
\dot{r}_{i h} \stackrel{*}{\rightarrow} \rho_{i} \text { weakly- } * \text { in } L^{\infty}(A) \text { for some } \rho \in L^{\infty}(A) \text {. }
$$

At the same time, since $r_{i}$ is locally Lipschitz on $A$, the function $r_{i} \in W^{1, \infty}(A)$ for every $i=1, \ldots, N$. Therefore, $\rho_{i}=\dot{r}_{i}$ for every $i=1, \ldots, N$, where $\dot{r}_{i}$ is the derivative of $r_{i}$ in the sense of distributions. Passing to a limit in Eqs. (121) and (122), we find that $r_{i}$ is a weak solution of

$$
\dot{r}_{i}+\frac{1}{r_{i}}-\lambda=0 \quad \text { if } d_{r}\left(r_{i}(t), t\right)>0
$$

and

$$
\dot{r}_{i}+\frac{1}{r_{i}}+\lambda=0 \quad \text { if } d_{r}\left(r_{i}(t), t\right)<0
$$

where $i=1, \ldots, N$.

Multiplying Eqs. (119) by $-r_{i h}$ and Eqs. (120) by $r_{i h}$ for their respective $i$ 's and adding the resulting equations together, we have for every $h>0$ that

$$
-\sum_{d_{r}>0} r_{i h} \dot{r}_{i h}+\sum_{d_{r}<0} r_{i h} \dot{r}_{i h}+\left(\sum_{i=1}^{N} r_{i h}\right) \lambda_{h}=a_{N}
$$

where $a_{N}$ is as in (124). Integrating this expression over $\left[t_{1}, t_{2}\right]$ for any $t_{1}, t_{2} \in A$ we conclude that for every $h>0$

$$
\int_{t_{1}}^{t_{2}}\left(-\sum_{d_{r}>0} r_{i h} \dot{r}_{i h}+\sum_{d_{r}<0} r_{i h} \dot{r}_{i h}\right) d t+\int_{t_{1}}^{t_{2}}\left(\sum_{i=1}^{N} r_{i h}\right) \lambda_{h} d t=\int_{t_{1}}^{t_{2}} a_{N} d t=a_{N}\left(t_{2}-t_{1}\right)
$$


since $a_{N}$ is constant on $A$. Therefore, using the mass preservation property of the limiting flow

$$
\int_{\Omega} v(t, x) d x=\int_{\Omega} v(0, x) d x \quad \text { for all } t \geq 0,
$$

we have that as $h \rightarrow 0$,

$$
\int_{t_{1}}^{t_{2}}\left(\sum_{i=1}^{N} r_{i}\right) \lambda d t=a_{N}\left(t_{2}-t_{1}\right) \quad \text { for any } t_{1}, t_{2} \in A \text {. }
$$

Since $\gamma$ in the statement of the previous theorem is arbitrary, "regular" interfaces evolve according to (121-122) on the interval $\left[t_{0}, t_{0}+\alpha\left(t_{0}\right)\right)$. Notice that in the previous theorem we can allow for the collisions or splittings of "ghost" interfaces on the interval $\left[t_{0}, t_{0}+\alpha\left(t_{0}\right)\right)$ as long as only new "ghost" interfaces appear as a result of these interactions.

REMARK 21. Since, for "ghost" interfaces, (117) and (118) do not hold, we are not able to determine their dynamics using our viscosity method.

7.4. Interactions of interfaces. In order to obtain a complete description of the interactions between interfaces, one has to identify $\lambda$ explicitly. This can be done by using the procedure of Lemma 6, which would require, in general, determining an asymptotic limit of the quantity

$$
\frac{1}{\varepsilon} \int_{0}^{R} W^{\prime}\left(u^{\varepsilon}\right)\left|u_{r}^{\varepsilon}\right| r d r=\frac{1}{\varepsilon^{2}} \int_{0}^{R} q\left(z^{\varepsilon} / \varepsilon\right)\left(q^{\prime}\left(z^{\varepsilon} / \varepsilon\right)\right)^{2}\left|z_{r}^{\varepsilon}\right| r d r .
$$

Unfortunately, this would require much sharper results on convergence of $z^{\varepsilon}$ to its asymptotic limit, since for the general initial data $u^{\varepsilon}$ may fail to be monotone near the interfaces. The task of proving these results is rather difficult and is beyond the scope of this paper. However, in some special cases the necessity of estimating (125) can be avoided. We return again to the problem studied at the end of Sec. 6.3.

Recall that $\tau$ is the first time when two "regular" interfaces collide or one "regular" interface shrinks to zero. Assume the former and let $\tau \in[0, T] \backslash \Pi_{\delta}$. Suppose without loss of generality that $a_{N}>0$, that all interfaces are located away from the center of the domain $\Omega$ and that the "ghost" interface, created as a result of the collision, does not disappear instantly. Then, by our assumption on $\tau$, there exists a number $\tau_{1}$ such that every interface is continuous on $\left[\tau, \tau_{1}\right]$. By Theorem 24 for any $t_{1}, t_{2} \in\left(\tau, \tau_{1}\right)$ we have

$$
\lim _{h \rightarrow 0} \int_{t_{1}}^{t_{2}}\left(\sum_{i=1}^{N-2} r_{i h}\right) \lambda_{h} d t=\int_{t_{1}}^{t_{2}}\left(\sum_{i=1}^{N-2} r_{i}\right) \lambda d t=a_{N}\left(t_{2}-t_{1}\right)
$$

where $N$ is the number of the "regular" interfaces at the time $t=0$.

On the other hand, we can estimate $\lambda_{h}$ using Lemma 6 and the inequality

$$
\liminf _{\varepsilon \rightarrow 0} \frac{1}{\varepsilon} \int_{0}^{R} W^{\prime}\left(u^{\varepsilon}(r, t)\right)\left|u_{r}^{\varepsilon}(r, t)\right| r d r \geq a_{N}(t),
$$


that can be proven using integration by parts. Then

$$
\lambda_{h} \geq \frac{a_{N}}{h} \int_{t}^{t+h}\left(p(s)+\sum_{i=1}^{N-2} r_{i}(s)\right)^{-1} d s
$$

where $r_{i}(t)$ is a position of the $i$ th "regular" interface for $i=1, \ldots, N$ and $p(t)$ is a position of the "ghost" interface. Substituting (127) to the left-hand side of (126), using the fact that $r_{i}(t)$ and $p(t)$ are bounded away from zero on $\left[\tau, \tau_{1}\right]$ for $i=1, \ldots, N$ and letting $h \rightarrow 0$ we find that

$$
a_{N}\left(t_{2}-t_{1}\right)<a_{N} \int_{t_{1}}^{t_{2}}\left(\sum_{i=1}^{N-2} r_{i}(s)\right)\left(p(s)+\sum_{i=1}^{N-2} r_{i}(s)\right)^{-1} d t \leq a_{N}\left(t_{2}-t_{1}\right)
$$

which is a contradiction. Therefore, there is no "ghost" interface past the time $t=\tau$. Moreover, since $\tau \in[0, T] \backslash \Pi_{\delta}$, we can use Remark 16 to show that one of the zeroes of $z_{r}^{\varepsilon}$ will disappear after the time $\tau$ for every $\varepsilon>0$ small. Hence we can continue applying the procedure of Sec. 6.3 to determine the evolution of the remaining interfaces.

This and some additional results can be proven for a general initial data by using the comparison arguments. We would like to prove

Theorem 25. For every $t \in[0, T] \backslash \Pi$, "regular" interfaces cannot split up into the interfaces of the lower multiplicity.

Proof. Let $t_{0} \in[0, T] \backslash \Pi$. Then there exists $\alpha\left(t_{0}\right)>0$ such that the limiting energy $E$ is Lipschitz continuous on the interval $\left[t_{0}, t_{0}+\alpha\left(t_{0}\right)\right]$. Suppose that $s\left(t_{0}\right)$ is a position of some "regular" interface of the multiplicity $m\left(t_{0}\right)>1$ such that $v$ increases across $s\left(t_{0}\right)$. We know that for a fixed $\delta>0$ small, the solution $u^{\varepsilon}\left(\cdot, t_{0}\right)$ of the nonlocal GinzburgLandau equation has exactly $m\left(t_{0}\right)$ zeroes inside the interval $\left[s\left(t_{0}\right)-\delta, s\left(t_{0}\right)+\delta\right]$ if $\varepsilon>0$ is small enough. For every small $\varepsilon>0$ choose the functions $\bar{\varphi}^{\varepsilon}$ and $\underline{\varphi}^{\varepsilon}$ in $C^{3}(\Omega)$ such that

$$
\bar{\varphi}^{\varepsilon}(r)=\underline{\varphi}^{\varepsilon}(r)=u^{\varepsilon}\left(r, t_{0}\right)
$$

if $r \notin\left[s\left(t_{0}\right)-\delta, s\left(t_{0}\right)+\delta\right]$ and

$$
1>\bar{\varphi}^{\varepsilon}(r) \geq u^{\varepsilon}\left(r, t_{0}\right) \geq \underline{\varphi}^{\varepsilon}(r)>-1
$$

if $r \in\left[s\left(t_{0}\right)-\delta, s\left(t_{0}\right)+\delta\right]$. Suppose, in addition, that both $\bar{\varphi}^{\varepsilon}$ and $\underline{\varphi}^{\varepsilon}$ satisfy (14.a-14.c) and that $\bar{\varphi}^{\varepsilon}$ has no more than one local maximum inside $\left(s\left(t_{0}\right)-\bar{\delta}, s\left(t_{0}\right)+\delta\right)$, while $\underline{\varphi}^{\varepsilon}$ has no more than one local minimum inside $\left(s\left(t_{0}\right)-\delta, s\left(t_{0}\right)+\delta\right)$. Then, clearly,

$$
\bar{\varphi}^{\varepsilon}, \underline{\varphi}^{\varepsilon} \rightarrow v\left(\cdot, t_{0}\right)
$$

in $L^{1}([0, R])$ and the multiplicities of the "regular" interfaces $\bar{s}\left(t_{0}\right)$ and $\underline{s}\left(t_{0}\right)$ are equal to one. Here $\bar{s}\left(t_{0}\right)$, corresponding to the asymptotic limit of $\bar{\varphi}^{\varepsilon}$, and $\underline{s}\left(t_{0}\right)$, corresponding to the asymptotic limit of $\underline{\varphi}^{\varepsilon}$, satisfy $\bar{s}\left(t_{0}\right)=\underline{s}\left(t_{0}\right)=s\left(t_{0}\right)$. 
Assume now that $\lambda^{\varepsilon}(t)$ is still determined by Eq. (19), evaluated at $u^{\varepsilon}(\cdot, t)$. We solve the following problems for $(x, t) \in \Omega \times\left[t_{0}, T\right]$ :

$$
\left(\begin{array}{lr}
\bar{u}_{t}^{\varepsilon}=\Delta \bar{u}^{\varepsilon}-\frac{1}{\varepsilon^{2}} f\left(\bar{u}^{\varepsilon}\right)+\frac{1}{\varepsilon} g\left(\bar{u}^{\varepsilon}\right) \lambda^{\varepsilon}, & x \in \Omega, t>t_{0}, \\
\left.\frac{\partial \bar{u}^{\varepsilon}}{\partial \mathbf{n}}\right|_{\partial \Omega}=0, & x \in \partial \Omega, \\
\bar{u}^{\varepsilon}\left(x, t_{0}\right)=\bar{\varphi}^{\varepsilon}(x), & x \in \Omega, t=t_{0},
\end{array}\right.
$$

and

$$
\left(\begin{array}{lr}
\underline{u}_{t}^{\varepsilon}=\Delta \underline{u}^{\varepsilon}-\frac{1}{\varepsilon^{2}} f\left(\underline{u}^{\varepsilon}\right)+\frac{1}{\varepsilon} g\left(\underline{u}^{\varepsilon}\right) \lambda^{\varepsilon}, & x \in \Omega, t>t_{0}, \\
\left.\frac{\partial \underline{u}^{\varepsilon}}{\partial \mathbf{n}}\right|_{\partial \Omega}=0, & x \in \partial \Omega, \\
\underline{u}^{\varepsilon}\left(x, t_{0}\right)=\underline{\varphi}^{\varepsilon}(x), & x \in \Omega, t=t_{0} .
\end{array}\right.
$$

Then, by the maximum principle,

$$
\underline{u}^{\varepsilon}(r, t) \leq u^{\varepsilon}(r, t) \leq \bar{u}^{\varepsilon}(r, t)
$$

for every $(r, t) \in[0, R] \times[0, \infty)$. Multiply Eq. (129) by $\bar{u}_{t}^{\varepsilon}$ and integrate over $\Omega$ by parts to obtain

$$
E^{\varepsilon}\left[\bar{u}^{\varepsilon}(\cdot, t)\right]=E^{\varepsilon}\left[\bar{\varphi}^{\varepsilon}\right]+\int_{t_{0}}^{t} \int_{\Omega} \lambda^{\varepsilon} h^{\prime}\left(\bar{u}^{\varepsilon}\right) \bar{u}_{t}^{\varepsilon} d x d s-\int_{t_{0}}^{t} \int_{\Omega} \varepsilon\left(\bar{u}_{t}^{\varepsilon}\right)^{2} d x d s
$$

Then, by Hölder's inequality and the definition of $h^{\prime}$,

$$
\begin{aligned}
E^{\varepsilon}\left[\bar{u}^{\varepsilon}(\cdot, t)\right] & \leq C^{\varepsilon}+2 \sqrt{2} \int_{t_{0}}^{t}\left|\lambda^{\varepsilon}\right|\left(\int_{\Omega} \frac{W\left(\bar{u}^{\varepsilon}\right)}{\varepsilon} d x\right)^{\frac{1}{2}}\left(\int_{\Omega} \varepsilon\left(\bar{u}_{t}^{\varepsilon}\right)^{2} d x\right)^{\frac{1}{2}} d s-\int_{t_{0}}^{t} \int_{\Omega} \varepsilon\left(\bar{u}_{t}^{\varepsilon}\right)^{2} d x d s \\
& \leq C^{\varepsilon}+\Lambda^{\varepsilon}(t)\left(E^{\varepsilon}\left[\bar{u}^{\varepsilon}(\cdot, t)\right]\right)^{\frac{1}{2}}\left(\int_{t_{0}}^{t} \int_{\Omega} \varepsilon\left(\bar{u}_{t}^{\varepsilon}\right)^{2} d x d s\right)^{\frac{1}{2}}-\int_{t_{0}}^{t} \int_{\Omega} \varepsilon\left(\bar{u}_{t}^{\varepsilon}\right)^{2} d x d s,
\end{aligned}
$$

where $C^{\varepsilon}$ depends only on the initial data and $\Lambda^{\varepsilon}(t)=2 \sqrt{2}\left(\int_{t_{0}}^{t}\left(\lambda^{\varepsilon}\right)^{2} d s\right)^{1 / 2}$. By Theorem 18 , we can choose for a small $\sigma>0$ a number $\beta\left(t_{0}\right)$ such that the function $\Lambda^{\varepsilon}(t)$ is less than $2-\sigma$ on the interval $\left[t_{0}, t_{0}+\beta\left(t_{0}\right)\right] \subset\left[t_{0}, t_{0}+\alpha\left(t_{0}\right)\right]$ uniformly in $\varepsilon$ and $t$. Then, by (132), we have that $E^{\varepsilon}\left[\bar{u}^{\varepsilon}(\cdot, t)\right] \leq \frac{4}{4 \sigma-\sigma^{2}} C^{\varepsilon}<\frac{4}{4 \sigma-\sigma^{2}} M$ uniformly in $\varepsilon$, where $M$ is given by (22). Moreover, by the same inequality $(132)$, we obtain that $E^{\varepsilon}\left[\bar{u}^{\varepsilon}(\cdot, t)\right]$ are bounded in $B V\left(\left[t_{0}, t_{0}+\beta\left(t_{0}\right)\right]\right)$ uniformly in $\varepsilon$. The same conclusions hold for the problem (130).

Hence, we can analyze both problems (129) and (130) using the methods developed in this paper. In particular, it turns out that the asymptotic limit of $\bar{u}^{\varepsilon}$ contains the interface $\bar{s}$ with the initial position at $\bar{s}\left(t_{0}\right)$, evolving according to the equation

$$
\dot{\bar{s}}+\frac{1}{\bar{s}}-\lambda=0
$$


on the interval $\left[t_{0}, t_{0}+\beta\left(t_{0}\right)\right]$, where $\lambda$ is defined in Theorem 24. At the same time the asymptotic limit of $\underline{u}^{\varepsilon}$ contains the interface $\underline{s}$ with the initial position at $\underline{s}\left(t_{0}\right)$ that evolves according to the same equation (133)

$$
\underline{\dot{s}}+\frac{1}{\underline{s}}-\lambda=0
$$

on the interval $\left[t_{0}, t_{0}+\beta\left(t_{0}\right)\right]$. By (131) we also have

$$
\underline{s}(t) \leq s(t) \leq \bar{s}(t)
$$

for every $t \in\left[t_{0}, t_{0}+\beta\left(t_{0}\right)\right]$. Equation (133) is obtained as a weak-* limit of Eq. (119) (cf. Theorem 24). Also the solutions of (119) converge uniformly to $\bar{s}$ on $t \in\left[t_{0}, t_{0}+\beta\left(t_{0}\right)\right]$. Therefore, we conclude that

$$
\int_{t_{0}}^{t_{0}+\beta\left(t_{0}\right)}\left(-\bar{s} \dot{\xi}+\frac{1}{\bar{s}} \xi-\lambda \xi\right) d t-\bar{s}\left(t_{0}\right) \xi\left(t_{0}\right)=0
$$

for every $\xi \in C^{1}\left(\left[t_{0}, t_{0}+\beta\left(t_{0}\right)\right]\right)$ such that $\xi\left(t_{0}+\beta\left(t_{0}\right)\right)=0$. Similarly, we have for $\underline{s}$

$$
\int_{t_{0}}^{t_{0}+\beta\left(t_{0}\right)}\left(-\underline{s} \dot{\xi}+\frac{1}{\underline{s}} \xi-\lambda \xi\right) d t-\underline{s}\left(t_{0}\right) \xi\left(t_{0}\right)=0
$$

for every $\xi \in C^{1}\left(\left[t_{0}, t_{0}+\beta\left(t_{0}\right)\right]\right)$ such that $\xi\left(t_{0}+\beta\left(t_{0}\right)\right)=0$. Then, since $\bar{s}\left(t_{0}\right)=\underline{s}\left(t_{0}\right)$, we obtain

$$
\int_{t_{0}}^{t_{0}+\beta\left(t_{0}\right)}\left((\bar{s}-\underline{s}) \dot{\xi}+\frac{(\bar{s}-\underline{s})}{\bar{s} \underline{s}} \xi\right) d t=0 .
$$

On the interval $\left[t_{0}, t_{0}+\beta\left(t_{0}\right)\right]$, the function $\bar{s} \underline{s} \geq a>0$ by the comparison (131) and the fact that $\left[t_{0}, t_{0}+\beta\left(t_{0}\right)\right] \subset\left[t_{0}, t_{0}+\alpha\left(t_{0}\right)\right] \subset[0, T] \backslash \Pi$. Then one can find a $C^{1}$-solution of

$$
\begin{gathered}
\dot{\xi}+\frac{1}{\bar{s} \underline{s}} \xi=\bar{s}-\underline{s}, \\
\xi\left(t_{0}+\beta\left(t_{0}\right)\right)=0,
\end{gathered}
$$

which implies, by (135), that $\bar{s}=\underline{s}$ on $\left[t_{0}, t_{0}+\beta\left(t_{0}\right)\right]$. Therefore, by (134), the "regular" interface $s$ cannot split up on the interval $\left[t_{0}, t_{0}+\beta\left(t_{0}\right)\right]$. Since $s$ and $t_{0}$ are arbitrary, no "regular" interface can split up into the interfaces of lower multiplicity for any $t \in$ $[0, T] \backslash \Pi$.

Suppose for simplicity that there are no interfaces in $[R-\gamma, R]$ at $t=0$ for some small $\gamma>0$. Then, by Remark 18, there are no interfaces inside the same interval for every $t>0$. Hence the approximate limit

$$
\text { ap } \lim _{r \rightarrow R} v(r, t) \equiv c
$$

is independent of time (for the definition of the approximate limit see, for example, [27]). Here $c=1$ or -1 . Assume without loss of generality that $c=1$. Then $a_{N}(t)$ will remain 
nonnegative for all $t>0$. Then we can use comparison arguments, similar to the ones given in Theorem 25, and nonnegativity of $a_{N}$ to deduce that

(a) the "ghost" interfaces from $N_{t}^{\delta}$ cannot exist on any interval in $[0, T] \backslash \Pi$ on which $a_{N}$ is strictly positive, that is, when the number of "regular" interfaces is odd.

(b) If a "ghost" interface from $P_{t}^{\delta}$ splits up into two "regular" interfaces at some time $t_{0}>0$, the latter interfaces will not collide with each other for any $t>t_{0}$.

(c) "Ghost" interfaces cannot split up on any interval in $[0, T] \backslash \Pi$ on which $a_{N}=0$, that is, when the number of "regular" interfaces is even.

Due to the lack of space we omit the proofs of these results, which can be shown by constructing the appropriate comparison functions.

The structure of Eqs. (121) and (122) allows us to conclude that the "ghost" interfaces that appear after the collisions of "regular" interfaces at some $t>0$ should belong to the set $N_{t}^{\delta}$ and therefore have to disappear when $a_{N}$ is strictly positive. In addition, one can see from (121) and (122) that no more than two "regular" interfaces can collide at the same point. The comparison arguments, however, do not allow us to determine whether the "ghost" interfaces from $P_{t}^{\delta}$ may exist for any period of time; otherwise, they might either disappear or split up. Of course, if the initial data is such that $a_{N}(t)$ is nonpositive for every $t>0$, then one should interchange $N_{t}^{\delta}$ and $P_{t}^{\delta}$ in the statements above.

On the basis of these results and the finiteness of the set $\Pi$, we find that the times, corresponding to the geometric singularities of the nonlocal flow, are contained in a finite subset $\Pi_{s}$ of $\mathbf{R}^{+}$. Here $\Pi_{s}$ consists of $\Pi$ and the times at which the interfaces collide or split up with the limiting energy remaining continuous. We, however, do not include in $\Pi_{s}$ those $t \in[0, \infty)$ when only "ghost" interfaces collide or split up creating, in turn, "ghost" interfaces only.

Fix any $t_{0} \in[0, T] \backslash \Pi_{s}$. Then there exists an $\alpha\left(t_{0}\right)>0$ such that the positions of "regular" interfaces on $\left[t_{0}, t_{0}+\alpha\left(t_{0}\right)\right]$ are continuous functions of $t$, uniformly bounded away from zero. Thus we can apply Theorem 24 to conclude that "regular" interfaces evolve by (121-122) on $\left[t_{0}, t_{0}+\alpha\left(t_{0}\right)\right)$. Since $t_{0} \in[0, T] \backslash \Pi_{s}$ can be chosen arbitrarily, we determine that on $[0, T] \backslash \Pi_{s}$ "regular" interfaces evolve by the weak nonlocal mean curvature flow $(121-122)$.

Choose $\tau$ such that $\tau>t$ for every $t \in \Pi_{s}$. Suppose that the number of "regular" interfaces at $\tau$ is even. Since the limiting energy $E$ is continuous on $[\tau, \infty)$, we have from (121) and (122) that "regular" interfaces evolve by curvature flow on $[\tau, \infty)$. Then the innermost of these interfaces will eventually shrink to the center of $\Omega$ for some $t>\tau$. Thus $t \in \Pi_{s}$. This contradicts our assumptions on $\tau$. So we deduce that the number of "regular" interfaces at $\tau$ should be odd. Then either $a_{N}(t)>0$ for all $t>\tau$ or $a_{N}(t)<0$ for all $t>\tau$. If there is more than one "regular" interface, we find by (121) and (122) that at some $t>\tau$ two "regular" interfaces will collide and disappear. Therefore, the limiting energy $E$ is discontinuous at $t$ and $t \in \Pi_{s}$ which, once again, contradicts the assumptions on $\tau$. Combining these observations with the fact that the volume must be preserved in the nonlocal flow, we obtain for $t>\tau$ that the asymptotic limit of solutions of the nonlocal Ginzburg-Landau equation can be characterized by a single stationary 
"regular" interface inside $\Omega$ such that

$$
\int_{\Omega} v(x, t) d x=\int_{\Omega} v(x, 0) d x .
$$

Depending on the sign of $a_{N}(t)$, this limit might contain "ghost" interfaces from either $N_{t}^{\delta}$ or $P_{t}^{\delta}$ as well.

We summarize these results in the following theorem.

TheOREM 26. Suppose that for some $t_{0} \geq 0$ there are finitely many interfaces. Suppose that $a_{N}\left(t_{0}\right)>0$ (or $\left.a_{N}\left(t_{0}\right)>0\right)$. Then there exists a finite set $\Pi_{s} \subset\left[t_{0}, \infty\right)$ such that

(a) "regular" interfaces evolve by the nonlocal curvature flow (121) and (122) on $\left[t_{0}, \infty\right) \backslash \Pi_{s}$

(b) $N_{t}^{\delta}=\varnothing$ (or $\left.P_{t}^{\delta}=\varnothing\right)$ for any $t \in\left[t_{0}, \infty\right) \backslash \Pi_{s}$ for which the number of "regular" interfaces is odd;

(c) if $t_{0}$ is such that $t_{0}>\sigma$ for every $\sigma \in \Pi_{s}$, then for every $t>t_{0}$ there exists exactly one stationary "regular" interface in $\Omega$ such that

$$
\int_{\Omega} v(x, t) d x=\int_{\Omega} v(x, 0) d x
$$

(d) the interfaces can collide, disappear, split up, change their multiplicity, or shrink to the center of $\Omega$ only at the times from the set $\Pi_{s}$, except the collisions or splittings of "ghost" interfaces that produce new "ghost" interfaces only.

If $a_{N}\left(t_{0}\right)=0$, then one has to substitute $a_{N}\left(t_{0}\right)$ in the statement of the previous theorem by ap $\lim _{r \rightarrow R} v(r, t)$. Notice also that the analog of Theorem 26 for the spaces of dimension higher than two will be slightly different due to the different formula for the Lagrange multiplier $\lambda$.

Finally, we conjecture that some of the results of the previous theorem can, in fact, be sharpened. Namely we suggest that "ghost" interfaces do not exist at the time $t$ if the energy is continuous at $t$. At least formally we can explain this conjecture as follows. One may think of a "ghost" interface as being formed by a pair of "regular" interfaces with the opposite sign jumps (for example, one interface is a jump from -1 to +1 and another from +1 to -1 ). From (121) and (122), we know that these interfaces will move with a different speed if $\lambda$ is not equal to zero. As a result, the "ghost" interface will have to either disappear or split up into two "regular" interfaces. Also, since the "regular" interface of multiplicity higher than one can be thought of as being formed by a "regular" interface of multiplicity one and a "ghost" interface, the same formal analysis suggests that multiplicity of the "regular" interface should not exceed one. However, to prove this fact rigorously, at least in the framework of our method, one has to study the limit of $(125)$ as $\varepsilon \rightarrow 0$.

\section{BIBLIOGRAPHY}

[1] J. Rubinstein and P. Sternberg, Nonlocal reaction-diffusion equations and nucleation, IMA J. Appl. Math. 48, 249-264 (1992)

[2] A. Novick-Cohen, On the viscous Cahn-Hilliard equation, Material Instabilities in Continuum Mechanics and Related Mathematical Problems (J. Ball, ed.), Clarendon Press, 1988, pp. 329-342 
[3] G. Barles, H. M. Soner, and P. E. Souganidis, Front propagation and phase field theory, SIAM J. Control Optim., March 1993, issue dedicated to W. H. Fleming

[4] L. Bronsard and B. Stoth, Volume preserving mean curvature flow as a limit of nonlocal GinzburgLandau equation (1994) (to appear)

[5] E. Giusti, Minimal Surfaces and Functions of Bounded Variation, Birkhaüser, Boston, 1984

[6] L. Bronsard and R. Kohn, Motion by mean curvature as the singular limit of the Ginzburg-Landau model, J. Differential Equations 90, 211-237 (1991)

[7] L. Modica, Gradient theory of phase transitions and the minimal interface criteria, Arch. Rat. Mech. Anal. 98, 123-142 (1987)

[8] P. Sternberg, The effect of a singular perturbation on nonconvex variational problems, Arch. Rat. Mech. Anal. 101, 209-260 (1988)

[9] I. Fonseca and L. Tartar, The gradient theory of phase transitions for systems with two potential wells, Proc. Royal Soc. Edinburgh Sect. A 111, 89-102 (1989)

[10] S. B. Angenent, The zero set of a solution of a parabolic equation, J. für die reine und angewandte Math. 390, 79-96 (1988)

[11] M. Protter and H. Weinberger, Maximum Principles in Differential Equations, Prentice-Hall, Englewood Cliffs, N. J., 1967

[12] B. Stoth, The Stefan problem with the Gibbs-Thompson law as singular limit of phase-field equations in the radial case, European J. Appl. Math. (1992) (to appear)

[13] H. M. Soner, Ginzburg-Landau equation and motion by mean curvature, I: Convergence, preprint, 1993

[14] L. Evans and J. Spruck, Motion of the level sets by mean curvature III, J. Geom. Analysis 2, $121-150(1992)$

[15] T. Ilmanen, Convergence of the Allen-Cahn equation to the Brakke's motion by mean curvature, preprint, 1991

[16] K. A. Brakke, The Motion of the Surface by its Mean Curvature, Princeton University Press, Princeton, N.J., 1978

[17] I. P. Natanson, Theory of functions of a real variable, vol. 2, Ungar Publishing Company, New York, NY, 1955

[18] D. Hilhorst, E. Logak, and Y. Nishiura, Singular limit for an Allen-Cahn equation with a nonlocal term, preprint, 1994

[19] L. Evans and J. Spruck, Motion of the level sets by mean curvature I, J. Differential Geom. 33, 635-681 (1991)

[20] Y. G. Chen, Y. Giga, and S. Goto, Uniqueness and existence of viscosity solutions of generalized mean curvature flow equations, J. Differential Geom. 33, 749-786 (1991)

[21] M. Crandall, H. Ishii, and P.-L. Lions, User's guide to viscosity solutions of second order partial differential equations, Bull. Amer. Math. Soc. 27, 1-67 (1992)

[22] G. Caginalp, An analysis of a phase field model of a free boundary, Arch. Rat. Mech. Anal. 92, 205-245 (1986)

[23] E. Fried and M. Gurtin, Continuum phase transitions with an order parameter; accretion and heat conduction, preprint, 1992

[24] O. Penrose and P. Fife, Thermodynamically consistent models for the kinetics of phase transitions, Physica D 43, 44-62 (1990)

[25] X. Chen, Hele-Shaw problem and area-preserving, curve shortening motion, Arch. Rat. Mech. Anal. 123, 117-151 (1993)

[26] X. Chen, D. Hilhorst, and E. Logak, Asymptotic behavior of an Allen-Cahn equation with a nonlocal term, preprint, 1994

[27] L. Evans and R. Gariepy, Measure Theory and Fine Properties of Functions, CRC Press, Boca Raton, 1992 